\title{
Generation and Intermolecular Capture of Cyclopropylacyl Radicals
}

\author{
Markus R. Heinrich and Samir Z. Zard*
}

Laboratoire de Synthèse Organique associé au CRNS, Ecole Polytechnique, 91128 Palaiseau Cedex, France

\section{General experimental}

Solvents and reagents were used as received. Infra-red spectra were recorded on a Fourier transform IR spectrometer. ${ }^{1} \mathrm{H}$ NMR were recorded using a $400 \mathrm{MHz}$ spectrometer using $\mathrm{CDCl}_{3}$ as solvent referenced to TMS (0 ppm) or $\mathrm{CHCl}_{3}(7.26 \mathrm{ppm})$. Chemical shifts are reported in parts per million (ppm). Coupling constants are in Hertz $(J \mathrm{~Hz})$; The following abbreviations are used for the description of signals: s (singlet), d (doublet), dd (double doublet), $\mathrm{t}$ (triplet), q (quadruplet), m (multiplet). ${ }^{13} \mathrm{C}$ NMR were recorded at $100.7 \mathrm{MHz}$ in $\mathrm{CDCl}_{3}$ using $\mathrm{CDCl}_{3}(77.0 \mathrm{ppm})$ as standard. Chemical shifts are given in parts per million (ppm). Mass spectra were recorded using electron impact (EI) and electron spray ionization (ESI). Analytical TLC was carried out on Merck silica gel plates using short wave (254 nm) UV light, $\mathrm{KMnO}_{4}$ and $\left(\mathrm{NH}_{4}\right)_{2} \mathrm{Ce}\left(\mathrm{NO}_{3}\right)_{6}$ to visualise components. Silica gel (Silice 60, A C.C 40-63 $\mu \mathrm{m}, \mathrm{SDS}$ ) was used for flash column chromatography. 
Dithiocarbonic acid [2-(1,3-dioxo-1,3-dihydroisoindol-2-yl)-2-methyl-propionyl] ester ethyl ester (3)

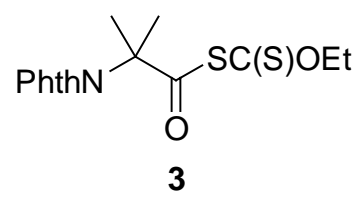

To a solution of 2-(1,3-dioxo-1,3-dihydro-isoindol-2-yl)-2-methyl-propionyl chloride $\mathbf{2}^{1}$ (1.00 $\mathrm{g}, 3.97 \mathrm{mmol})$ in acetone $(20 \mathrm{~mL})$ at $0^{\circ} \mathrm{C}$ was added ethyl xanthic acid potassium salt $(0.61 \mathrm{~g}$, $3.81 \mathrm{mmol}$ ). After stirring for $15 \mathrm{~min}$ at $0^{\circ} \mathrm{C}$ the solvent was removed under reduced pressure at $0-10^{\circ} \mathrm{C}$. The residue was dissolved in dichloromethane, washed with water, followed by brine and dried over $\mathrm{Na}_{2} \mathrm{SO}_{4}$. Evaporation of the solvent gave acyl xanthate $\mathbf{3}$ (1.20 g, 3.56 mmol, 94\%)

$R_{\mathrm{f}}: \quad 0.60(1: 4$ EtOAc-hexanes $)$

IR: $\quad\left(\mathrm{CCl}_{4}\right): \mathrm{v} ;^{\sim}=1781(\mathrm{~m}), 1738(\mathrm{~m}), 1725(\mathrm{~s}), 1264(\mathrm{~m}, \mathrm{C}-\mathrm{S}), 1041(\mathrm{~m}, \mathrm{C}=\mathrm{S})$ $\mathrm{cm}^{-1}$.

${ }^{1} \mathrm{H}-\mathrm{NMR}: \quad\left(400 \mathrm{MHz}, \mathrm{CDCl}_{3}\right): \delta=1.46\left(\mathrm{t},{ }^{3} J=7.0 \mathrm{~Hz}, 3 \mathrm{H}\right), 1.86(\mathrm{~s}, 6 \mathrm{H}), 4.66\left(\mathrm{q},{ }^{3} J=\right.$ $7.0 \mathrm{~Hz}, 2 \mathrm{H}), 7.72-7.76(\mathrm{~m}, 2 \mathrm{H}), 7.80-7.84(\mathrm{~m}, 2 \mathrm{H})$.

${ }^{13} \mathrm{C}-\mathrm{NMR}: \quad\left(100.7 \mathrm{MHz}, \mathrm{CDCl}_{3}\right): \delta=13.33\left(\mathrm{CH}_{3}\right), 24.12\left(2 \times \mathrm{CH}_{3}\right), 66.92\left(\mathrm{C}_{\mathrm{q}}\right), 70.98$ $\left(\mathrm{CH}_{2}\right), 123.37(2 \times \mathrm{CH}), 131.35\left(2 \times \mathrm{C}_{\mathrm{q}}\right), 134.38(2 \times \mathrm{CH}), 167.95\left(2 \times \mathrm{C}_{\mathrm{q}}\right)$, $192.10\left(\mathrm{C}_{\mathrm{q}}\right), 203.39\left(\mathrm{C}_{\mathrm{q}}\right)$.

ESI-MS: $\quad 354\left(\mathrm{M}^{+}+\mathrm{NH}_{3}\right), 338\left(\mathrm{M}^{+}+\mathrm{H}\right)$

$\mathrm{C}_{15} \mathrm{H}_{15} \mathrm{NO}_{4} \mathrm{~S}_{2}(337.42)$

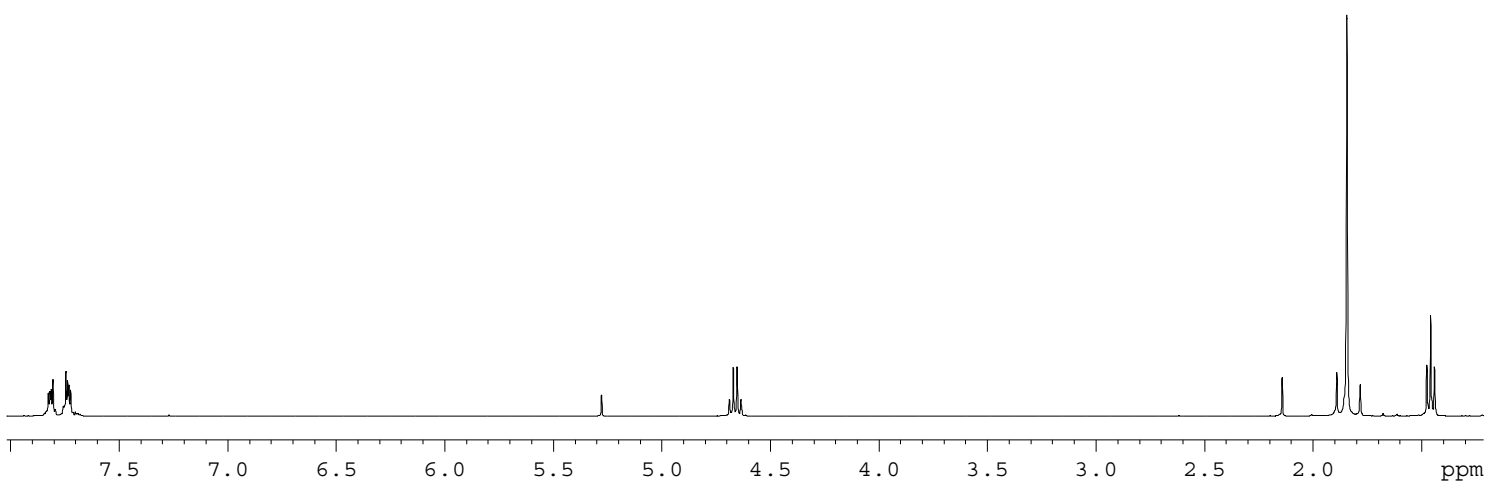




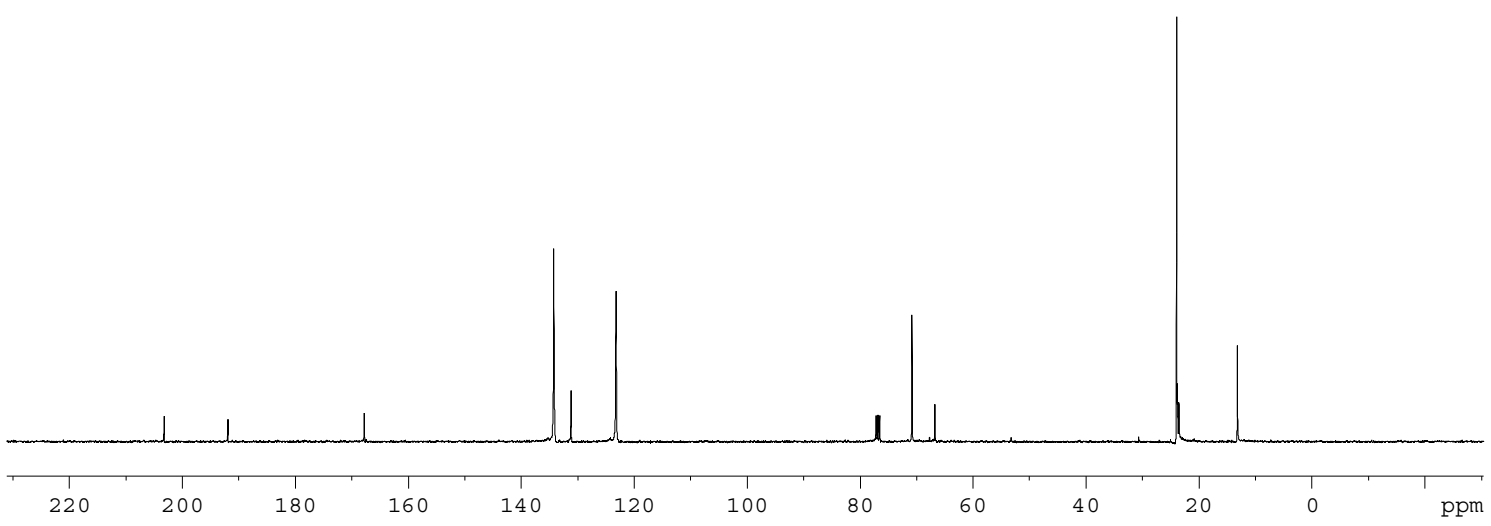

2-tert-Butoxycarbonylamino-6-(1,3-dioxo-1,3-dihydro-isoindol-2-yl)-4-ethoxythiocarbonylsulfanyl-6-methyl-heptanoic acid methyl ester (8)

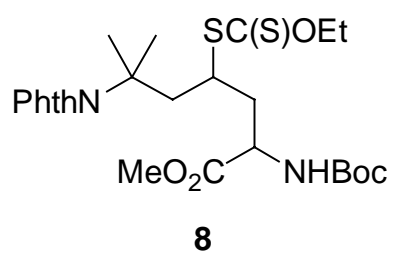

A degassed solution of xanthate $3(0.30 \mathrm{~g}, 0.89 \mathrm{mmol})$, 2-tert-butoxycarbonylamino-pent-4enoic acid methyl ester $4^{2}(0.10 \mathrm{~g}, 0.44 \mathrm{mmol})$ and DLP $(0.18 \mathrm{~g}, 0.44 \mathrm{mmol})$ in 1,2dichloroethane $(1.0 \mathrm{~mL})$ was stirred for $5 \mathrm{~h}$ at $60^{\circ} \mathrm{C}$. The mixture was concentrated under reduced pressure and the residue was purified by column chromatography (1:4 EtOAc-hexanes) to give the two separated isomers $\mathbf{8 a}$ and $\mathbf{8 b}(5+8 \mathrm{mg}, 0.02 \mathrm{mmol}, 6 \%)$.

$R_{\mathrm{f}}: \quad 0.45(\mathbf{8 a}), 0.35(\mathbf{8 b})(1: 4$ EtOAc-hexanes $)$

IR (8a): $\quad\left(\mathrm{CCl}_{4}\right): \mathrm{v} ;^{\sim}=1774(\mathrm{w}), 1746(\mathrm{~m}), 1712(\mathrm{~s}), 1220(\mathrm{~m}, \mathrm{C}-\mathrm{S}), 1055(\mathrm{~m}, \mathrm{C}=\mathrm{S})$ $\mathrm{cm}^{-1}$.

${ }^{1} \mathrm{H}-\mathrm{NMR}(\mathbf{8 a}): \quad\left(400 \mathrm{MHz}, \mathrm{CDCl}_{3}\right): \delta=1.27\left(\mathrm{t},{ }^{3} J=7.2 \mathrm{~Hz}, 3 \mathrm{H}\right), 1.44(\mathrm{~s}, 9 \mathrm{H}), 1.71(\mathrm{~s}, 3 \mathrm{H})$, $1.81(\mathrm{~s}, 3 \mathrm{H}), 1.95-2.07(\mathrm{~m}, 2 \mathrm{H}), 2.11-2.18(\mathrm{~m}, 1 \mathrm{H}), 2.79\left(\mathrm{dd},{ }^{3} J=15.6 \mathrm{~Hz}\right.$, $\left.{ }^{3} J=10.4 \mathrm{~Hz}, 1 \mathrm{H}\right), 3.69$ (s, 3H), 3.92-4.01 (m, 1H), 4.33-4.40 (m, 1H), 4.43 $\left(\mathrm{q},{ }^{3} J=7.2 \mathrm{~Hz}, 2 \mathrm{H}\right), 5.22\left(\mathrm{~d},{ }^{3} J=8.4 \mathrm{~Hz}, \mathrm{NH}\right), 7.62-7.86(\mathrm{~m}, 4 \mathrm{H})$.

${ }^{13} \mathrm{C}-\mathrm{NMR}(\mathbf{8 a}): \quad\left(100.7 \mathrm{MHz}, \mathrm{CDCl}_{3}\right): \delta=13.23\left(\mathrm{CH}_{3}\right), 26.52\left(\mathrm{CH}_{3}\right), 27.97\left(3 \times \mathrm{CH}_{3}\right), 28.74$ $\left(\mathrm{CH}_{3}\right), 39.48\left(\mathrm{CH}_{2}\right), 40.34\left(\mathrm{CH}_{2}\right), 43.05\left(\mathrm{CH} / \mathrm{CH}_{3}\right), 51.09\left(\mathrm{CH} / \mathrm{CH}_{3}\right), 52.27$ $\left(\mathrm{CH} / \mathrm{CH}_{3}\right), 59.29\left(\mathrm{C}_{\mathrm{q}}\right), 69.78\left(\mathrm{CH}_{2}\right), 80.11\left(\mathrm{C}_{\mathrm{q}}\right), 122.47(2 \times \mathrm{CH}), 131.76$ $\left(2 \times \mathrm{C}_{\mathrm{q}}\right), 133.63(2 \times \mathrm{CH}), 155.52\left(\mathrm{C}_{\mathrm{q}}\right), 169.97\left(2 \times \mathrm{C}_{\mathrm{q}}\right), 172.62\left(\mathrm{C}_{\mathrm{q}}\right), 212.70$ $\left(\mathrm{C}_{\mathrm{q}}\right)$. 


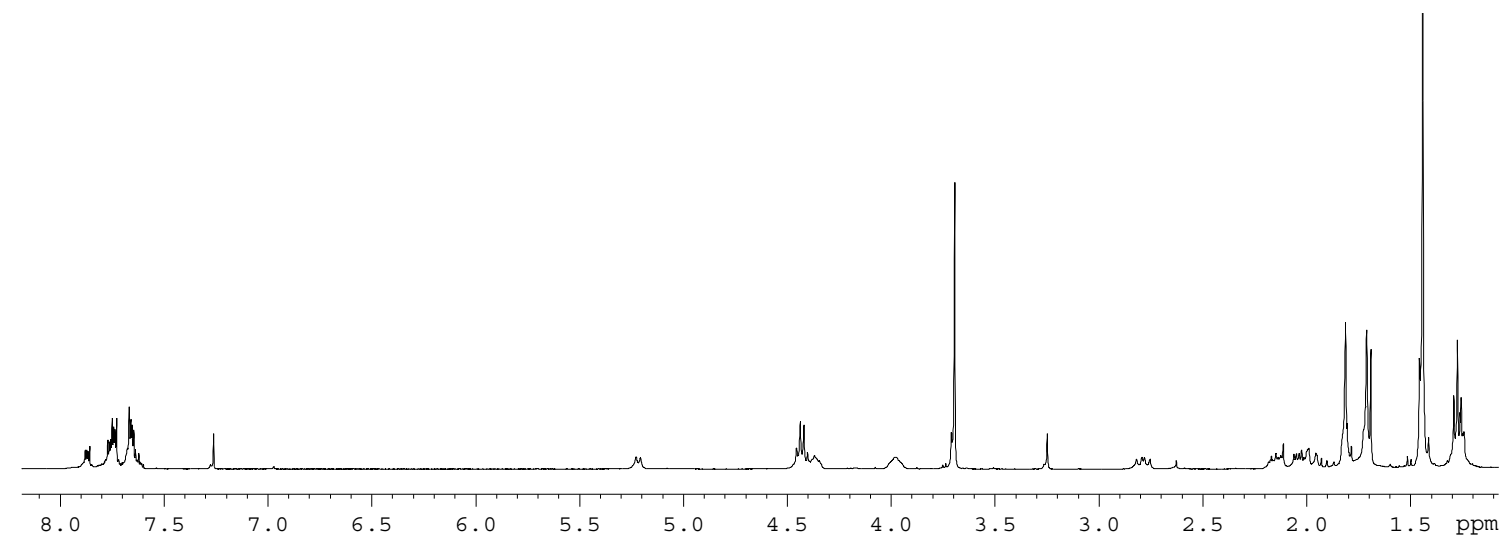

IR (8b): $\quad\left(\mathrm{CCl}_{4}\right): \mathrm{v} ;^{\sim}=1774(\mathrm{w}), 1746(\mathrm{~m}), 1712(\mathrm{~s}), 1216(\mathrm{~m}, \mathrm{C}-\mathrm{S}), 1055(\mathrm{~m}, \mathrm{C}=\mathrm{S})$ $\mathrm{cm}^{-1}$.

${ }^{1} \mathrm{H}-\mathrm{NMR}(\mathbf{8 b}): \quad\left(400 \mathrm{MHz}, \mathrm{CDCl}_{3}\right): \delta=1.26\left(\mathrm{t},{ }^{3} J=6.4 \mathrm{~Hz}, 3 \mathrm{H}\right), 1.41(\mathrm{~s}, 9 \mathrm{H}), 1.76(\mathrm{~s}, 3 \mathrm{H})$, $1.80(\mathrm{~s}, 3 \mathrm{H}), 1.99-2.09(\mathrm{~m}, 1 \mathrm{H}), 2.11-2.24(\mathrm{~m}, 2 \mathrm{H}), 2.60-2.68(\mathrm{~m}, 1 \mathrm{H})$, $3.68(\mathrm{~s}, 3 \mathrm{H}), 3.95-4.02(\mathrm{~m}, 1 \mathrm{H}), 4.30\left(\mathrm{dt},{ }^{3} \mathrm{~J}=8.0 \mathrm{~Hz},{ }^{3} \mathrm{~J}=6.8 \mathrm{~Hz}, 1 \mathrm{H}\right)$, 4.38-4.47 (m, 2H), $5.08\left(\mathrm{~d},{ }^{3} J=8.0 \mathrm{~Hz}, \mathrm{NH}\right), 7.50-7.76(\mathrm{~m}, 4 \mathrm{H})$.

${ }^{13} \mathrm{C}-\mathrm{NMR}(\mathbf{8 b}): \quad\left(100.7 \mathrm{MHz}, \mathrm{CDCl}_{3}\right): \delta=13.56\left(\mathrm{CH}_{3}\right), 27.75\left(\mathrm{CH}_{3}\right), 28.29\left(3 \times \mathrm{CH}_{3}\right), 28.82$ $\left(\mathrm{CH}_{3}\right), 39.61\left(\mathrm{CH}_{2}\right), 42.71\left(\mathrm{CH}_{2}\right), 44.08\left(\mathrm{CH} / \mathrm{CH}_{3}\right), 51.55\left(\mathrm{CH} / \mathrm{CH}_{3}\right), 52.36$ $\left(\mathrm{CH} / \mathrm{CH}_{3}\right), 59.71\left(\mathrm{C}_{\mathrm{q}}\right), 69.88\left(\mathrm{CH}_{2}\right), 79.96\left(\mathrm{C}_{\mathrm{q}}\right), 122.66(2 \times \mathrm{CH}), 132.09$ $\left(2 \times \mathrm{C}_{\mathrm{q}}\right), 133.70(2 \times \mathrm{CH}), 155.03\left(\mathrm{C}_{\mathrm{q}}\right), 169.90\left(2 \times \mathrm{C}_{\mathrm{q}}\right), 172.58\left(\mathrm{C}_{\mathrm{q}}\right), 212.70$ $\left(\mathrm{C}_{\mathrm{q}}\right)$.

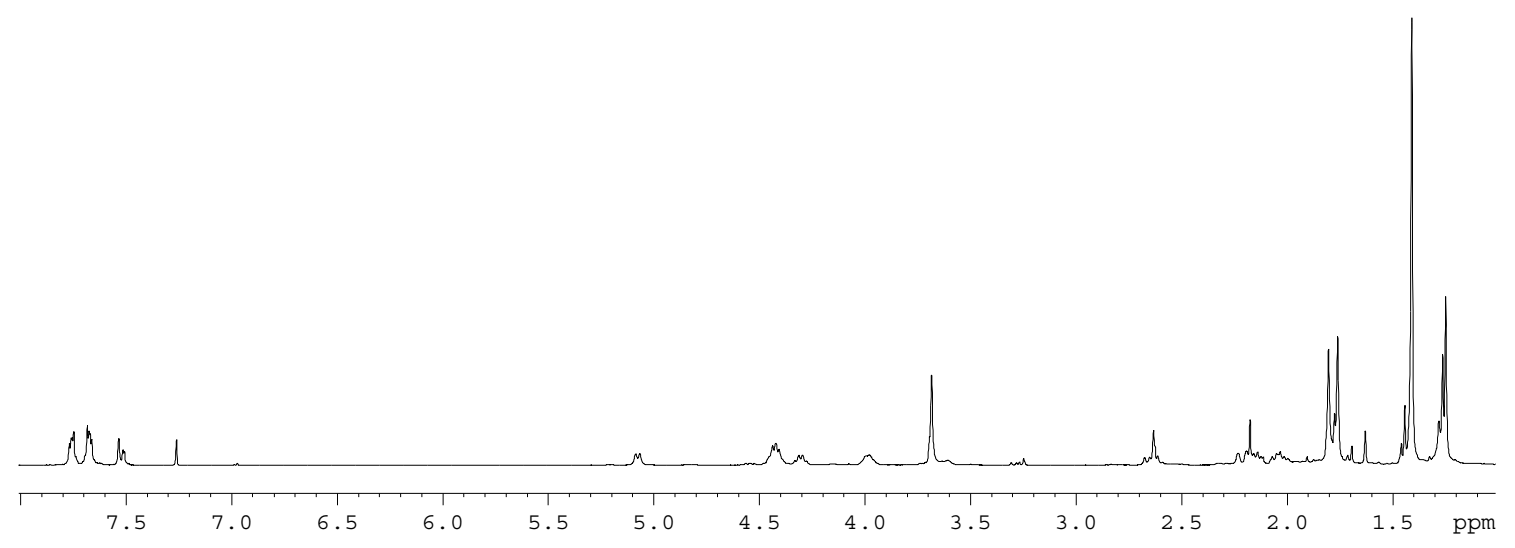

ESI-MS: $\quad 556\left(\mathrm{M}^{+}+\mathrm{NH}_{4}\right), 539\left(\mathrm{M}^{+}+\mathrm{H}\right)$.

FAB-HRMS: $\quad \mathrm{C}_{25} \mathrm{H}_{34} \mathrm{~N}_{2} \mathrm{O}_{7} \mathrm{~S}_{2} \mathrm{Na}^{+}$, calcd.: 561.1705, found: 561.1730 .

$\mathrm{C}_{25} \mathrm{H}_{35} \mathrm{~N}_{2} \mathrm{O}_{7} \mathrm{~S}_{2}{ }^{+}$, calcd.: 539.1886, found: 539.1865. 


$$
\mathrm{C}_{25} \mathrm{H}_{34} \mathrm{~N}_{2} \mathrm{O}_{7} \mathrm{~S}_{2}(538.68)
$$

\section{Acetic acid 4-(1,3-dioxo-1,3-dihydro-isoindol-2-yl)-2-ethoxythiocarbonylsulfanyl-4-}

methyl-pentyl ester (9)

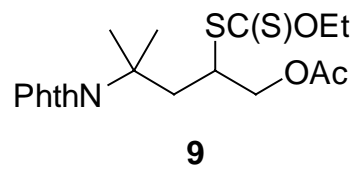

A degassed solution of xanthate $3(454 \mathrm{mg}, 1.35 \mathrm{mmol})$ in allyl acetate $(2.50 \mathrm{~mL})$ was exposed to visible light for $10 \mathrm{~h}$ whilst the reaction temperature was kept below $40^{\circ} \mathrm{C}$. The solvent was removed under reduced pressure and the residue was purified by column chromatography (1:4 EtOAc-hexanes) to give 9 (420 mg, $1.03 \mathrm{mmol}, 76 \%$ ).

Yield: $\quad 76 \%$

$R_{\mathrm{f}}: \quad 0.40(1: 4$ EtOAc-hexanes $)$

IR: $\quad\left(\mathrm{CCl}_{4}\right): \mathrm{v} ;{ }^{\sim}=1775(\mathrm{~m}), 1747(\mathrm{~s}), 1713(\mathrm{~s}), 1225(\mathrm{~m}, \mathrm{C}-\mathrm{S}), 1054(\mathrm{~m}, \mathrm{C}=\mathrm{S})$ $\mathrm{cm}^{-1}$.

${ }^{1} \mathrm{H}-\mathrm{NMR}: \quad\left(400 \mathrm{MHz}, \mathrm{CDCl}_{3}\right): \delta=1.31\left(\mathrm{t},{ }^{3} \mathrm{~J}=6.8 \mathrm{~Hz}, 3 \mathrm{H}\right), 1.80(\mathrm{~s}, 3 \mathrm{H}), 1.81(\mathrm{~s}, 3 \mathrm{H})$, 1.99 (s, 3H), 2.33 (dd, $\left.{ }^{2} J=15.6 \mathrm{~Hz},{ }^{3} J=3.2 \mathrm{~Hz}, 1 \mathrm{H}\right), 2.43\left(\mathrm{dd},{ }^{2} J=15.6\right.$ $\left.\mathrm{Hz},{ }^{3} J=8.0 \mathrm{~Hz}, 1 \mathrm{H}\right), 4.08-4.20(\mathrm{~m}, 3 \mathrm{H}), 4.45-4.53(\mathrm{~m}, 2 \mathrm{H}), 7.66-7.71$ (m, $2 \mathrm{H}), 7.75-7.78(\mathrm{~m}, 2 \mathrm{H})$.

${ }^{13} \mathrm{C}-\mathrm{NMR}: \quad\left(100.7 \mathrm{MHz}, \mathrm{CDCl}_{3}\right): \delta=13.55\left(\mathrm{CH}_{3}\right), 20.70\left(\mathrm{CH}_{3}\right), 27.79\left(\mathrm{CH}_{3}\right), 28.18$ $\left(\mathrm{CH}_{3}\right), 39.06\left(\mathrm{CH}_{2}\right), 45.23(\mathrm{CH}), 59.67\left(\mathrm{C}_{\mathrm{q}}\right), 66.62\left(\mathrm{CH}_{2}\right), 70.07\left(\mathrm{CH}_{2}\right)$, $122.69(2 \times \mathrm{CH}), 131.95\left(2 \times \mathrm{C}_{\mathrm{q}}\right), 133.78(2 \times \mathrm{CH}), 169.79\left(2 \times \mathrm{C}_{\mathrm{q}}\right), 170.61\left(\mathrm{C}_{\mathrm{q}}\right)$, $212.21\left(\mathrm{C}_{\mathrm{q}}\right)$.

ESI-MS: $\quad 427\left(\mathrm{M}^{+}+\mathrm{NH}_{4}\right), 410\left(\mathrm{M}^{+}+\mathrm{H}\right), 408\left(\mathrm{M}^{+}-\mathrm{H}\right)$

EI-HRMS: $\quad \mathrm{C}_{19} \mathrm{H}_{23} \mathrm{NO}_{5} \mathrm{~S}_{2}$, calcd.: 409.1018, found: 409.1040 .

$\mathrm{C}_{19} \mathrm{H}_{23} \mathrm{NO}_{5} \mathrm{~S}_{2}(409.52)$ 

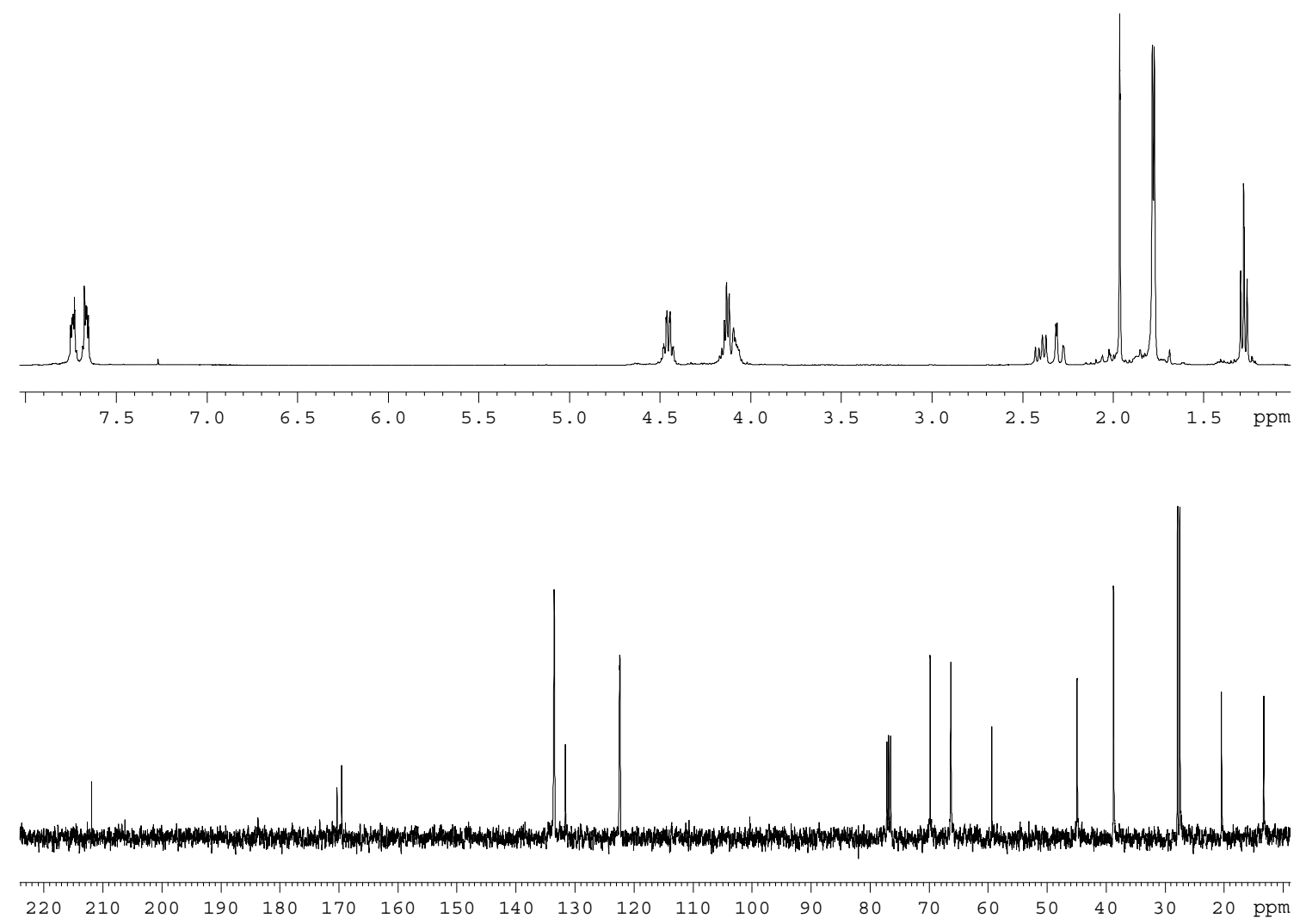

2,2-Dimethyl-propionic acid 3-(1,3-dioxo-1,3-dihydro-isoindol-2-yl)-1-ethoxythiocarbonylsulfanyl-3-methyl-butyl ester (10)

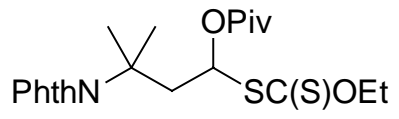

10

A degassed solution of xanthate $3(170 \mathrm{mg}, 0.50 \mathrm{mmol})$ in vinyl pivalate $(1.50 \mathrm{~mL})$ was exposed to visible light for $2 \mathrm{~h}$ while the reaction temperature was kept below $40^{\circ} \mathrm{C}$. The solvent was removed under reduced pressure and the residue was purified by column chromatography (1:10 EtOAc-hexanes) to give 10 (206 mg, $0.47 \mathrm{mmol}, 94 \%)$.

$R_{\mathrm{f}}: \quad 0.70(1: 4$ EtOAc-hexanes $)$

IR: $\quad\left(\mathrm{CCl}_{4}\right): v^{\sim}{ }^{\sim}=1775(\mathrm{~m}), 1735(\mathrm{~m}), 1714(\mathrm{~s}), 1224(\mathrm{~m}, \mathrm{C}-\mathrm{S}), 1050(\mathrm{~m}, \mathrm{C}=\mathrm{S})$ $\mathrm{cm}^{-1}$. 
${ }^{1} \mathrm{H}-\mathrm{NMR}: \quad\left(400 \mathrm{MHz}, \mathrm{CDCl}_{3}\right): \delta=1.01(\mathrm{~s}, 9 \mathrm{H}), 1.29\left(\mathrm{t},{ }^{3} J=7.2 \mathrm{~Hz}, 3 \mathrm{H}\right), 1.73(\mathrm{~s}, 3 \mathrm{H})$, $1.74(\mathrm{~s}, 3 \mathrm{H}), 2.67-2.70(\mathrm{~m}, 2 \mathrm{H}), 4.49(\mathrm{~m}, 2 \mathrm{H}), 6.66\left(\mathrm{dd},{ }^{3} \mathrm{~J}=6.8 \mathrm{~Hz},{ }^{3} \mathrm{~J}=\right.$ $5.6 \mathrm{~Hz}, 1 \mathrm{H}), 7.62-7.65(\mathrm{~m}, 2 \mathrm{H}), 7.68-7.72(\mathrm{~m}, 2 \mathrm{H})$.

${ }^{13} \mathrm{C}-\mathrm{NMR}: \quad\left(100.7 \mathrm{MHz}, \mathrm{CDCl}_{3}\right): \delta=14.02\left(\mathrm{CH}_{3}\right), 26.55\left(3 \times \mathrm{CH}_{3}\right), 27.64\left(\mathrm{CH}_{3}\right), 27.99$ $\left(\mathrm{CH}_{3}\right), 38.93\left(\mathrm{C}_{\mathrm{q}}\right), 43.96\left(\mathrm{CH}_{2}\right), 58.76\left(\mathrm{C}_{\mathrm{q}}\right), 69.88\left(\mathrm{CH}_{2}\right), 77.95(\mathrm{CH})$, $122.53(2 \times \mathrm{CH}), \quad 131.82\left(2 \times \mathrm{C}_{\mathrm{q}}\right), 133.69(2 \times \mathrm{CH}), 169.40\left(2 \times \mathrm{C}_{\mathrm{q}}\right), 176.18$ $\left(\mathrm{C}_{\mathrm{q}}\right), 209.64\left(\mathrm{C}_{\mathrm{q}}\right)$.

ESI-MS: $\quad 455\left(\mathrm{M}^{+}+\mathrm{NH}_{4}\right), 438\left(\mathrm{M}^{+}+\mathrm{H}\right)$

FAB-HRMS: $\quad \mathrm{C}_{21} \mathrm{H}_{27} \mathrm{NO}_{5} \mathrm{~S}_{2} \mathrm{Na}^{+}$, calcd.: 460.1229 , found: 460.1199 .

$\mathrm{C}_{21} \mathrm{H}_{27} \mathrm{NO}_{5} \mathrm{~S}_{2}$ (437.57)
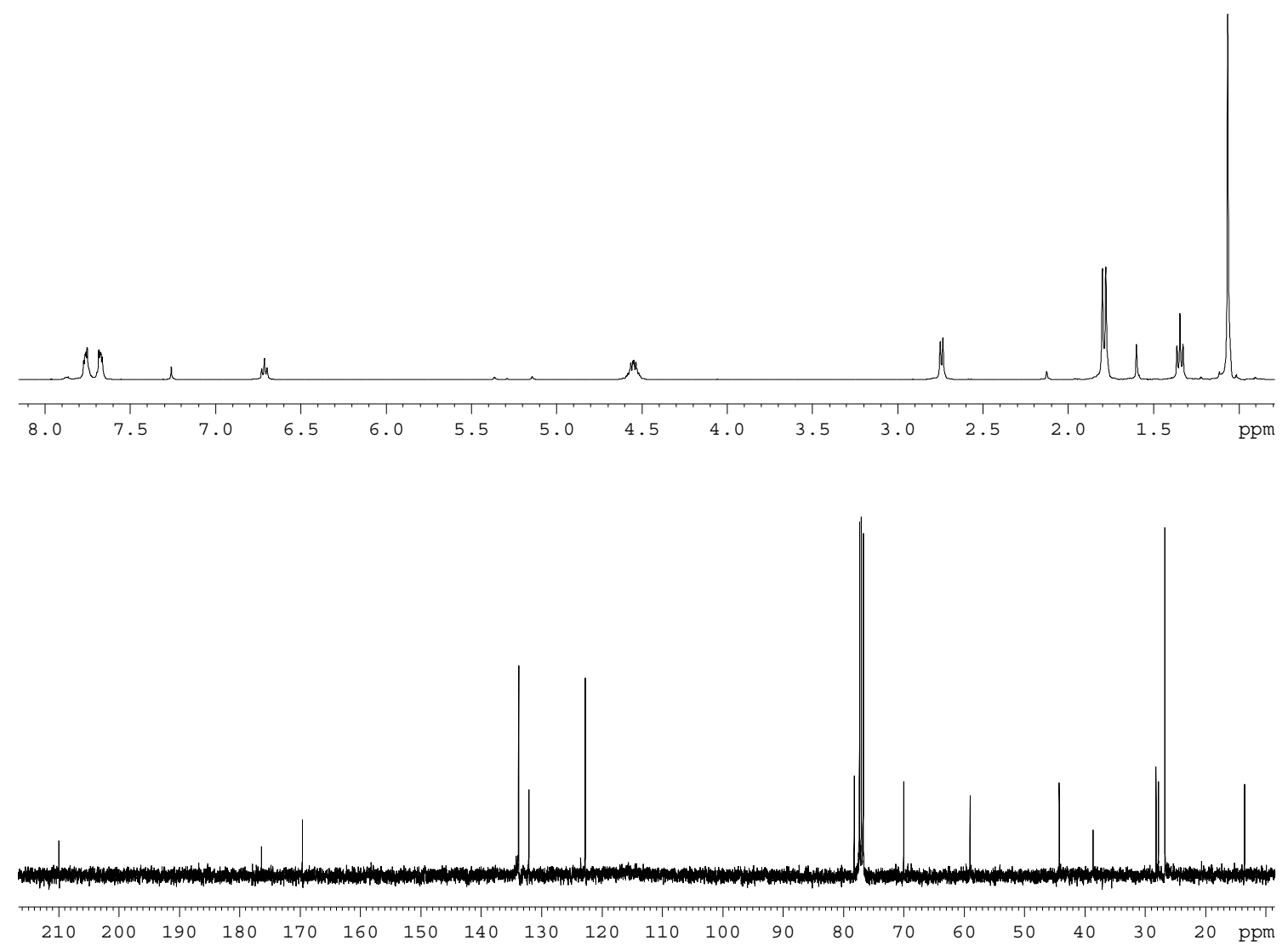
Dithiocarbonic acid [1-(1,3-dioxo-1,3-dihydroisoindol-2-yl)-cyclopropylacyl] ester ethyl ester (15)

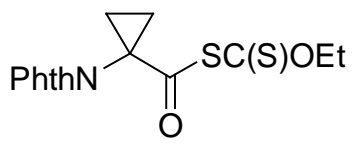

15

To a solution of 1-(1,3-dioxo-1,3-dihydro-isoindol-2-yl)-cyclopropanecarbonyl chloride $\mathbf{1 4}^{3}$ $(1.05 \mathrm{~g}, 4.20 \mathrm{mmol})$ in acetone $(20 \mathrm{~mL})$ at $0^{\circ} \mathrm{C}$ was added ethyl xanthic acid potassium salt $(0.61 \mathrm{~g}, 3.81 \mathrm{mmol})$. After stirring for $15 \mathrm{~min}$ at $0^{\circ} \mathrm{C}$ the solvent was removed under reduced pressure at $0-10^{\circ} \mathrm{C}$. The residue was dissolved in dichloromethane, washed with water, followed by brine and dried over $\mathrm{Na}_{2} \mathrm{SO}_{4}$. Evaporation of the solvent gave acyl xanthate 15 (1.15 g, $3.43 \mathrm{mmol}, 90 \%)$

$R_{\mathrm{f}}: \quad 0.45(1: 4$ EtOAc-hexanes $)$

m.p.: $\quad 116-118^{\circ} \mathrm{C}$

IR: $\quad\left(\mathrm{CCl}_{4}\right): \mathrm{v} ; ;^{\sim}=1786(\mathrm{~m}), 1735(\mathrm{~s}), 1391(\mathrm{~s}), 1264(\mathrm{~m}, \mathrm{C}-\mathrm{S}), 1042(\mathrm{~m}, \mathrm{C}=\mathrm{S})$ $\mathrm{cm}^{-1}$.

${ }^{1} \mathrm{H}-\mathrm{NMR}: \quad\left(400 \mathrm{MHz}, \mathrm{CDCl}_{3}\right): \delta=1.41\left(\mathrm{t},{ }^{3} J=7.2 \mathrm{~Hz}, 3 \mathrm{H}\right), 1.56\left(\mathrm{dd},{ }^{2} J=9.0 \mathrm{~Hz},{ }^{3} J=\right.$ $5.4 \mathrm{~Hz}, 2 \mathrm{H}), 1.95\left(\mathrm{dd},{ }^{2} J=9.0 \mathrm{~Hz},{ }^{3} J=5.4 \mathrm{~Hz}, 2 \mathrm{H}\right), 4.60\left(\mathrm{q},{ }^{3} J=7.2 \mathrm{~Hz}\right.$, 2H), 7.74-7.80 (m, 2H), 7.84-7.89 (m, 2H).

${ }^{13} \mathrm{C}-\mathrm{NMR}: \quad\left(100.7 \mathrm{MHz}, \mathrm{CDCl}_{3}\right): \delta=13.26\left(\mathrm{CH}_{3}\right), 19.78\left(2 \times \mathrm{CH}_{2}\right), 38.64\left(\mathrm{C}_{\mathrm{q}}\right), 71.09$ $\left(\mathrm{CH}_{2}\right), 123.74(2 \times \mathrm{CH}), 130.99\left(2 \times \mathrm{C}_{\mathrm{q}}\right), 134.59(2 \times \mathrm{CH}), 167.28\left(2 \times \mathrm{C}_{\mathrm{q}}\right)$, $190.26\left(\mathrm{C}_{\mathrm{q}}\right), 201.76\left(\mathrm{C}_{\mathrm{q}}\right)$.

ESI-MS: $\quad 352\left(\mathrm{M}^{+}+\mathrm{NH}_{3}\right), 335\left(\mathrm{M}^{+}\right)$.

$\mathrm{C}_{15} \mathrm{H}_{13} \mathrm{NO}_{4} \mathrm{~S}_{2}(335.40)$ 

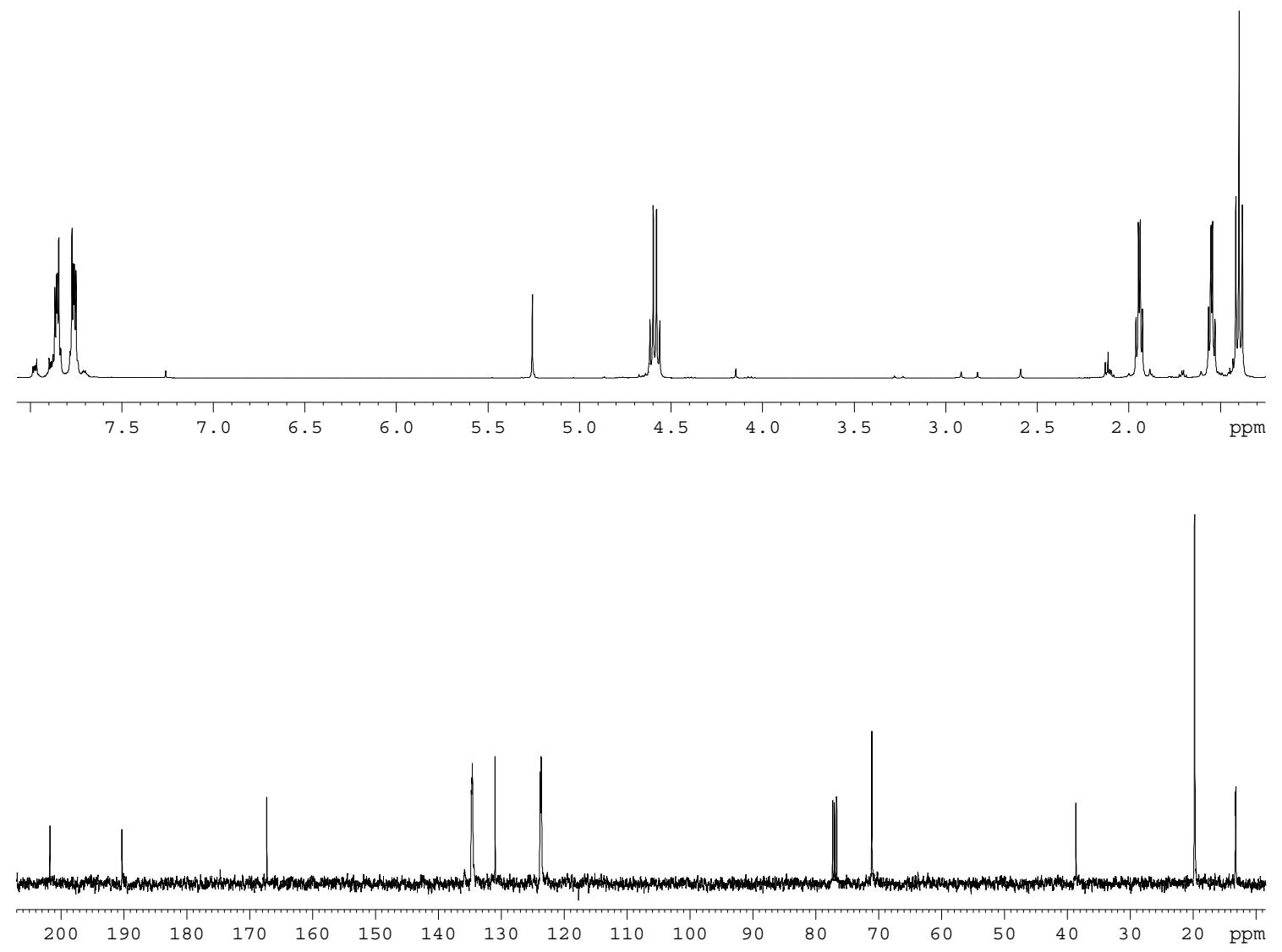

Acetic acid 4-[1-(1,3-dioxo-1,3-dihydro-isoindol-2-yl)-cyclopropyl]-2-ethoxythiocarbonylsulfanyl-4-oxo-butyl ester (20)<smiles></smiles>

20

A solution of xanthate $15(151 \mathrm{mg}, 0.45 \mathrm{mmol})$ and allyl acetate $(0.10 \mathrm{~mL}, 92 \mathrm{mg}, 0.92$ mmol) in 1,2-dichloroethane $(0.5 \mathrm{~mL})$ was refluxed for $5 \mathrm{~min}$ under argon. After the addition of DLP (18 mg, $0.05 \mathrm{mmol}, 10 \%)$ the mixture was refluxed for $1 \mathrm{~h}$ and concentrated under reduced pressure. Purification by column chromatography (1:4 EtOAc-hexanes) gave 20 (123 $\mathrm{mg}, 0.28 \mathrm{mmol}, 63 \%)$.

$R_{\mathrm{f}}:$

0.40 (1:4 EtOAc-hexanes)

IR:

$\left(\mathrm{CCl}_{4}\right): \mathrm{v}^{\sim}{ }^{\sim}=1782(\mathrm{~m}), 1749(\mathrm{~s}), 1728(\mathrm{~s}), 1395(\mathrm{~s}), 1227(\mathrm{~m}, \mathrm{C}-\mathrm{S}), 1053$ $(\mathrm{m}, \mathrm{C}=\mathrm{S}) \mathrm{cm}^{-1}$. 
${ }^{1} \mathrm{H}-\mathrm{NMR}: \quad\left(400 \mathrm{MHz}, \mathrm{CDCl}_{3}\right): \delta=1.41\left(\mathrm{t},{ }^{3} \mathrm{~J}=7.0 \mathrm{~Hz}, 3 \mathrm{H}\right), 1.48-1.51(\mathrm{~m}, 2 \mathrm{H})$, $1.89-1.92(\mathrm{~m}, 2 \mathrm{H}), 2.06$ (s, 3H), $2.84\left(\mathrm{dd},{ }^{2} J=18.0 \mathrm{~Hz},{ }^{3} J=5.6 \mathrm{~Hz}, 1 \mathrm{H}\right)$, $2.90\left(\mathrm{dd},{ }^{2} J=18.0 \mathrm{~Hz},{ }^{3} J=7.0 \mathrm{~Hz}, 1 \mathrm{H}\right), 4.19\left(\mathrm{dd},{ }^{2} J=11.4 \mathrm{~Hz},{ }^{3} J=6.0 \mathrm{~Hz}\right.$, $1 \mathrm{H}), 4.30\left(\mathrm{dd},{ }^{2} J=11.4 \mathrm{~Hz},{ }^{3} J=5.0 \mathrm{~Hz}, 1 \mathrm{H}\right), 4.35-4.41(\mathrm{~m}, 1 \mathrm{H}), 4.61\left(\mathrm{q},{ }^{3} J\right.$ $=7.0 \mathrm{~Hz}, 2 \mathrm{H}), 7.77-7.82(\mathrm{~m}, 2 \mathrm{H}), 7.88-7.91(\mathrm{~m}, 2 \mathrm{H})$.

${ }^{13} \mathrm{C}$-NMR: $\quad\left(100.7 \mathrm{MHz}, \mathrm{CDCl}_{3}\right): \delta=13.64\left(\mathrm{CH}_{3}\right), 19.05\left(\mathrm{CH}_{2}\right), 19.15\left(\mathrm{CH}_{2}\right), 20.69$ $\left(\mathrm{CH}_{3}\right), 38.75\left(\mathrm{CH}_{2} / \mathrm{C}_{\mathrm{q}}\right), 39.21\left(\mathrm{CH}_{2} / \mathrm{C}_{\mathrm{q}}\right), 43.45(\mathrm{CH}), 64.32\left(\mathrm{CH}_{2}\right), 70.29$ $\left(\mathrm{CH}_{2}\right), 123.71(2 \times \mathrm{CH}), 131.33\left(2 \times \mathrm{C}_{\mathrm{q}}\right), 134.61(2 \times \mathrm{CH}), 168.03\left(2 \times \mathrm{C}_{\mathrm{q}}\right)$, $170.52\left(\mathrm{C}_{\mathrm{q}}\right), 202.91\left(\mathrm{C}_{\mathrm{q}}\right), 212.16\left(\mathrm{C}_{\mathrm{q}}\right)$.

ESI-MS: $\quad 453\left(\mathrm{M}^{+}+\mathrm{NH}_{4}\right), 336\left(\mathrm{M}^{+}+\mathrm{H}\right)$.

EI-HRMS: $\quad \mathrm{C}_{20} \mathrm{H}_{21} \mathrm{NO}_{6} \mathrm{~S}_{2}$, calcd.: 435.0810, found: 435.0799. $\mathrm{C}_{20} \mathrm{H}_{21} \mathrm{NO}_{6} \mathrm{~S}_{2}$ (435.52)
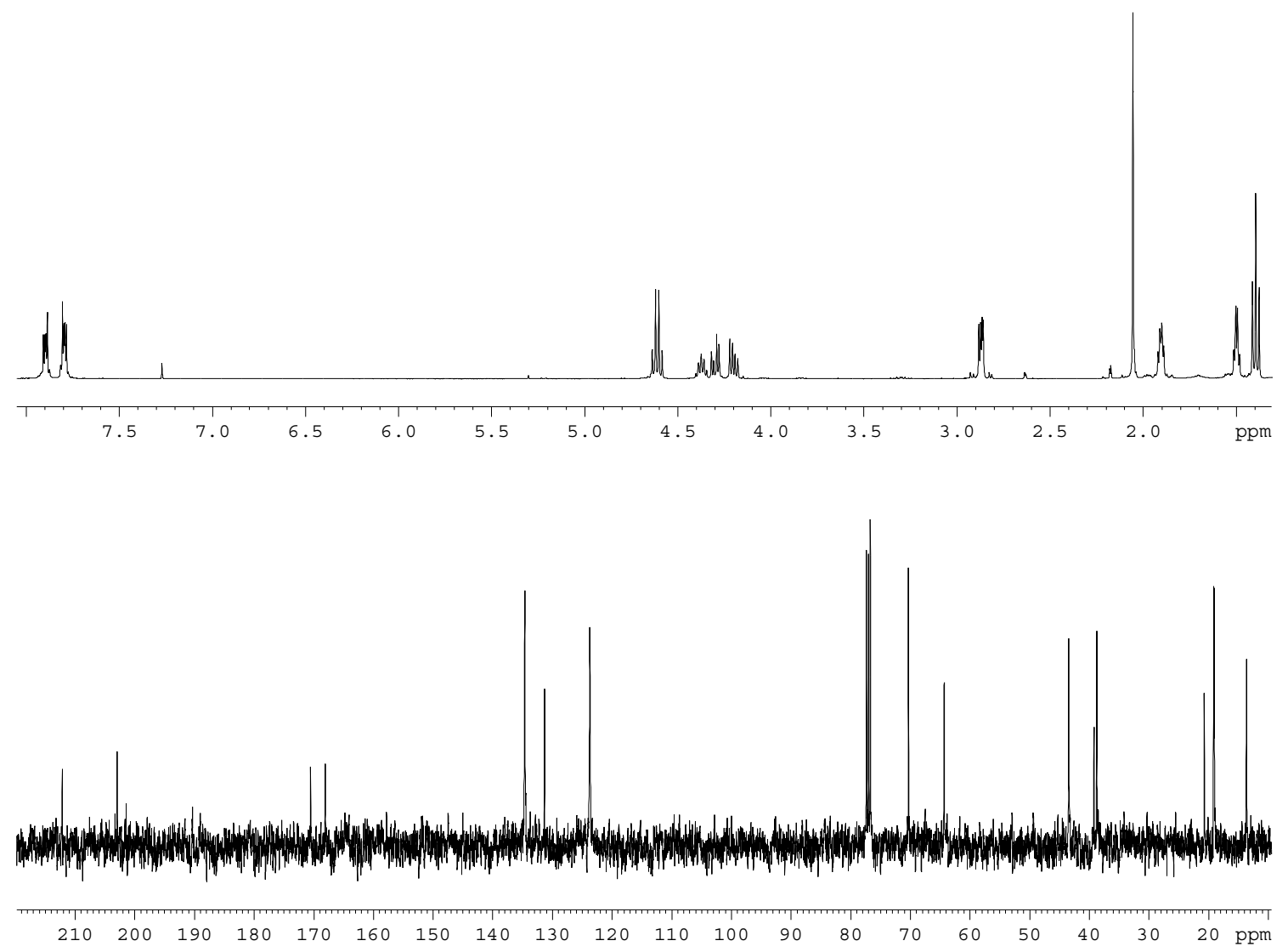


\section{Dithiocarbonic acid (1-\{2-[1-(1,3-dioxo-1,3-dihydro-isoindol-2-yl)-cyclopropyl]-2-oxo-}

ethyl\}-heptyl) ester ethyl ester (22)

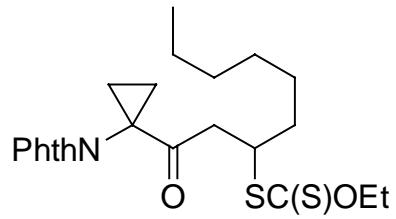

22

A solution of xanthate $15(168 \mathrm{mg}, 0.50 \mathrm{mmol})$ and 1-octene $(0.16 \mathrm{~mL}, 112 \mathrm{mg}, 1.00 \mathrm{mmol})$ in 1,2-dichloroethane $(0.5 \mathrm{~mL})$ was refluxed for 5 min under argon. After the addition of DLP (20 $\mathrm{mg}, 0.05 \mathrm{mmol}, 10 \%$ ) the mixture was refluxed for $2.5 \mathrm{~h}$ while one more portion of DLP (20 mg, $0.05 \mathrm{mmol}$ ) was added after $1 \mathrm{~h}$. Concentration under reduced pressure and purification by column chromatography (1:4 EtOAc-hexanes) gave 22 (137 mg, $0.31 \mathrm{mmol}$, $61 \%)$.

$R_{\mathrm{f}}: \quad 0.75(1: 4$ EtOAc-hexanes $)$

IR: $\quad\left(\mathrm{CCl}_{4}\right): \mathrm{v}^{\sim}=1782(\mathrm{~m}), 1728(\mathrm{~s}), 1710(\mathrm{~s}), 1394(\mathrm{~s}), 1218(\mathrm{~m}, \mathrm{C}-\mathrm{S}), 1053$ $(\mathrm{m}, \mathrm{C}=\mathrm{S}) \mathrm{cm}^{-1}$.

${ }^{1} \mathrm{H}-\mathrm{NMR}: \quad\left(400 \mathrm{MHz}, \mathrm{CDCl}_{3}\right): \delta=0.82\left(\mathrm{t},{ }^{3} \mathrm{~J}=6.8 \mathrm{~Hz}, 3 \mathrm{H}\right), 1.12-1.30(\mathrm{~m}, 8 \mathrm{H}), 1.35$ $\left(\mathrm{t},{ }^{3} J=7.2 \mathrm{~Hz}, 3 \mathrm{H}\right), 1.41-1.45(\mathrm{~m}, 2 \mathrm{H}), 1.45-1.60$ (m, 2H), 1.81-1.85 (m, 2H), $2.70\left(\mathrm{dd},{ }^{2} J=18.0 \mathrm{~Hz},{ }^{3} J=8.4 \mathrm{~Hz}, 1 \mathrm{H}\right), 2.98\left(\mathrm{dd},{ }^{2} J=18.0 \mathrm{~Hz},{ }^{3} \mathrm{~J}=\right.$ $4.0 \mathrm{~Hz}, 1 \mathrm{H}), 3.96-4.03(\mathrm{~m}, 1 \mathrm{H}), 4.55(\mathrm{~m}, 2 \mathrm{H}), 7.72-7.76(\mathrm{~m}, 2 \mathrm{H})$, $7.82-7.86(\mathrm{~m}, 2 \mathrm{H})$.

${ }^{13} \mathrm{C}-\mathrm{NMR}: \quad\left(100.7 \mathrm{MHz}, \mathrm{CDCl}_{3}\right): \delta=13.55\left(\mathrm{CH}_{3}\right), 13.91\left(\mathrm{CH}_{3}\right), 18.57\left(\mathrm{CH}_{2}\right), 18.72$ $\left(\mathrm{CH}_{2}\right), 22.37\left(\mathrm{CH}_{2}\right), 26.38\left(\mathrm{CH}_{2}\right), 28.72\left(\mathrm{CH}_{2}\right), 31.41\left(\mathrm{CH}_{2}\right), 32.99\left(\mathrm{CH}_{2}\right)$, $39.21\left(\mathrm{C}_{\mathrm{q}}\right), 42.73\left(\mathrm{CH}_{2}\right), 45.32(\mathrm{CH}), 69.67\left(\mathrm{CH}_{2}\right), 123.58(2 \times \mathrm{CH}), 131.29$ $\left(2 \times \mathrm{C}_{\mathrm{q}}\right), 134.44(2 \times \mathrm{CH}), 167.98\left(2 \times \mathrm{C}_{\mathrm{q}}\right), 203.47\left(\mathrm{C}_{\mathrm{q}}\right), 213.55\left(\mathrm{C}_{\mathrm{q}}\right)$.

EI-HRMS: $\quad \mathrm{C}_{23} \mathrm{H}_{29} \mathrm{NO}_{4} \mathrm{~S}_{2}$, calcd.: 447.1538, found: 447.1537.

$\mathrm{C}_{23} \mathrm{H}_{29} \mathrm{NO}_{4} \mathrm{~S}_{2}(447.61)$ 

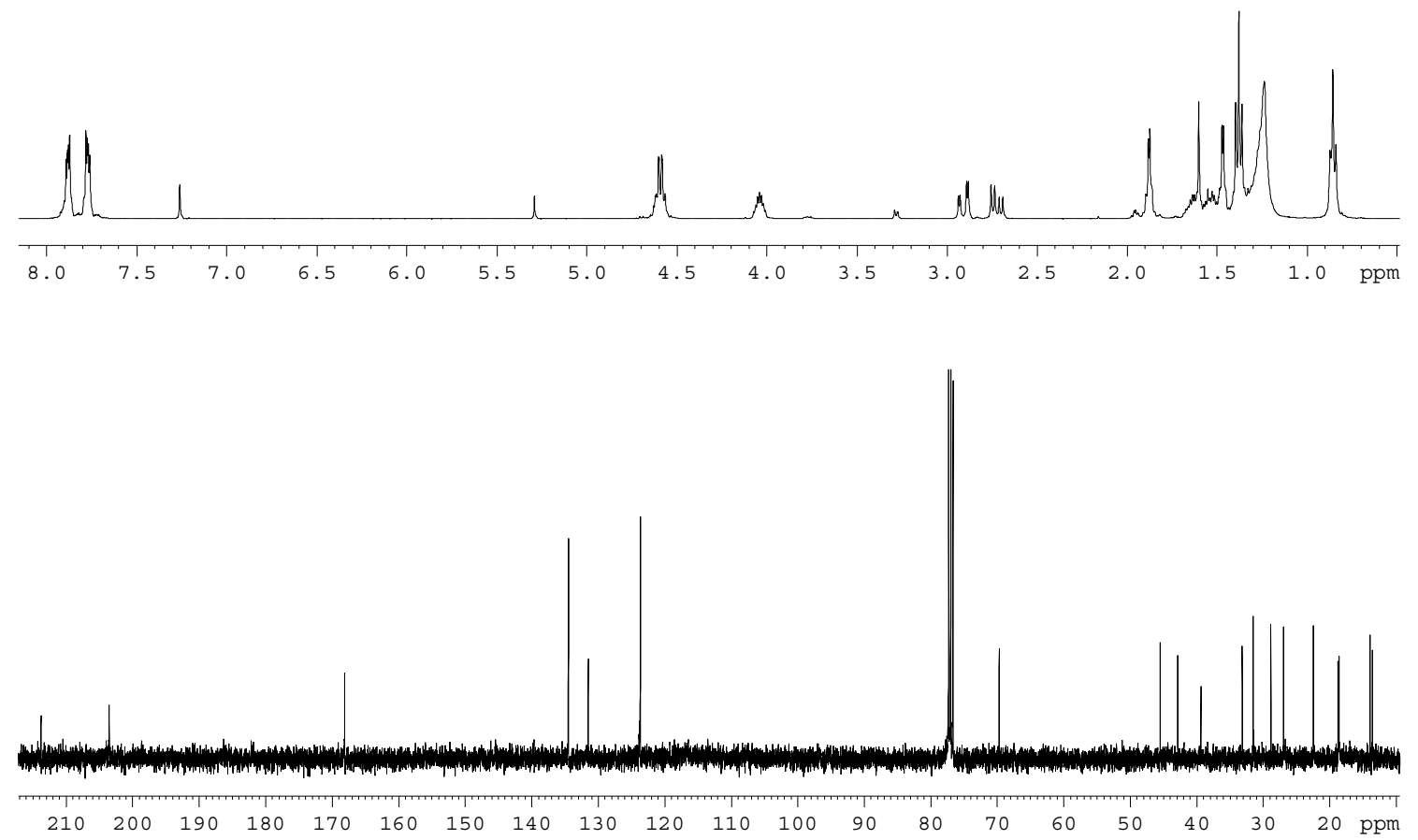

2,2-Dimethyl-propionic acid 3-[1-(1,3-dioxo-1,3-dihydro-isoindol-2-yl)-cyclopropyl]-1ethoxythiocarbonylsulfanyl-3-oxo-propyl ester (23)

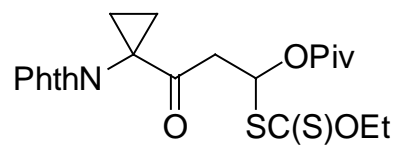

23

A solution of xanthate $15(168 \mathrm{mg}, 0.50 \mathrm{mmol})$ and vinyl pivalate $(0.15 \mathrm{~mL}, 131 \mathrm{mg}, 1.02$ mmol) in 1,2-dichloroethane $(1 \mathrm{~mL})$ was exposed to visible light and refluxed for $5 \mathrm{~h}$. Concentration and purification by column chromatography (1:10 EtOAc-hexanes) gave starting material 15 (50 mg, $0.15 \mathrm{mmol}, 30 \%$ ) followed by 23 (97 mg, $0.21 \mathrm{mmol}, 42 \%$ ).

$R_{\mathrm{f}}: \quad 0.20(1: 10$ EtOAc-hexanes $)$

IR: $\quad\left(\mathrm{CCl}_{4}\right): \mathrm{v}^{\sim}{ }^{\sim}=1782(\mathrm{~m}), 1729(\mathrm{~s}), 1395(\mathrm{~s}), 1225(\mathrm{~m}, \mathrm{C}-\mathrm{S}), 1135$ (s), 1053 (s, $\mathrm{C}=\mathrm{S}) \quad \mathrm{cm}^{-1}$.

${ }^{1} \mathrm{H}-\mathrm{NMR}: \quad\left(400 \mathrm{MHz}, \mathrm{CDCl}_{3}\right): \delta=1.12(\mathrm{~s}, 9 \mathrm{H}), 1.35\left(\mathrm{t},{ }^{3} \mathrm{~J}=7.2 \mathrm{~Hz}, 3 \mathrm{H}\right), 1.44-1.52$ (m, 2H), $1.82-1.93(\mathrm{~m}, 2 \mathrm{H}), 3.03\left(\mathrm{dd},{ }^{2} J=17.8 \mathrm{~Hz},{ }^{3} J=4.6 \mathrm{~Hz}, 1 \mathrm{H}\right), 3.10$ $\left(\mathrm{dd},{ }^{2} J=17.8 \mathrm{~Hz},{ }^{3} J=7.2 \mathrm{~Hz}, 1 \mathrm{H}\right), 4.56\left(\mathrm{q},{ }^{3} J=7.2 \mathrm{~Hz}, 2 \mathrm{H}\right), 6.77\left(\mathrm{dd},{ }^{3} J=\right.$ $\left.7.2 \mathrm{~Hz},{ }^{3} J=4.6 \mathrm{~Hz}, 1 \mathrm{H}\right), 7.68-7.79(\mathrm{~m}, 2 \mathrm{H}), 7.79-7.89(\mathrm{~m}, 2 \mathrm{H})$. 
${ }^{13} \mathrm{C}-\mathrm{NMR}: \quad\left(100.7 \mathrm{MHz}, \mathrm{CDCl}_{3}\right): \delta=13.54\left(\mathrm{CH}_{3}\right), 18.60\left(\mathrm{CH}_{2}\right), 18.66\left(\mathrm{CH}_{2}\right), 26.71$ $\left(3 \times \mathrm{CH}_{3}\right), 38.74\left(\mathrm{C}_{\mathrm{q}}\right), 39.25\left(\mathrm{C}_{\mathrm{q}}\right), 42.01\left(\mathrm{CH}_{2}\right), 70.13\left(\mathrm{CH}_{2}\right), 75.44(\mathrm{CH})$, $123.62(2 \times \mathrm{CH}), 131.25\left(2 \times \mathrm{C}_{\mathrm{q}}\right), 134.51(2 \times \mathrm{CH}), 167.91\left(2 \times \mathrm{C}_{\mathrm{q}}\right), 176.13\left(\mathrm{C}_{\mathrm{q}}\right)$, $200.90\left(\mathrm{C}_{\mathrm{q}}\right), 209.84\left(\mathrm{C}_{\mathrm{q}}\right)$.

ESI-MS: $\quad 481\left(\mathrm{M}^{+}+\mathrm{NH}_{4}\right), 463\left(\mathrm{M}^{+}\right)$.

EI-HRMS: $\quad \mathrm{C}_{22} \mathrm{H}_{25} \mathrm{NO}_{6} \mathrm{~S}_{2}$, calcd.: 463.1123, found: 463.1114 .

$$
\mathrm{C}_{22} \mathrm{H}_{25} \mathrm{NO}_{6} \mathrm{~S}_{2} \text { (463.57) }
$$
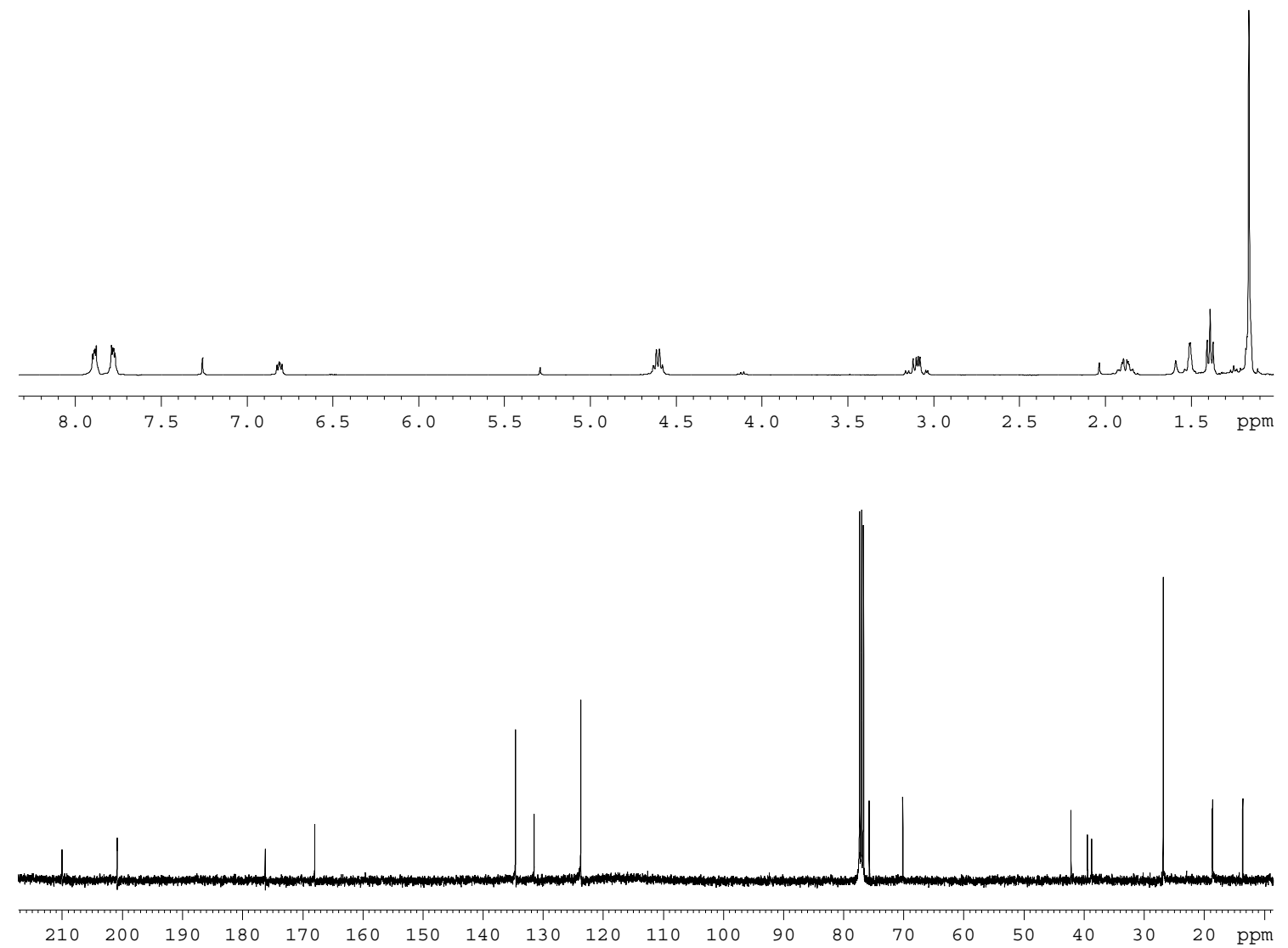

2-tert-Butoxycarbonylamino-6-[1-(1,3-dioxo-1,3-dihydro-isoindol-2-yl)-cyclopropyl]-4ethoxythiocarbonylsulfanyl-6-oxo-hexanoic acid methyl ester (24), mixture of isomers<smiles>CCO[Si]C(CC(=O)C1(Nc2ccccc2)CC1)CC(NC(C)(C)C)C(=O)OCC</smiles> 
A solution of xanthate $15(180 \mathrm{mg}, 0.54 \mathrm{mmol})$ and 2-tert-butoxycarbonylamino-pent-4-enoic acid methyl ester $\mathbf{4}^{2}(185 \mathrm{mg}, 0.80 \mathrm{mmol})$ in 1,2-dichloroethane $(1.0 \mathrm{~mL})$ was refluxed for 5 mins under argon. After the addition of DLP (21 mg, $0.05 \mathrm{mmol}, 10 \%)$ the mixture was refluxed for $2 \mathrm{~h}$ while two more portions of DLP (11 $\mathrm{mg}, 0.03 \mathrm{mmol})$ were added. Concentration under reduced pressure and purification by column chromatography (3:7 EtOAc-hexanes) gave 24 (173 mg, $0.31 \mathrm{mmol}, 57 \%)$.

$R_{\mathrm{f}}: \quad 0.45(3: 7$ EtOAc-hexanes $)$

IR: $\quad\left(\mathrm{CCl}_{4}\right): \mathrm{v}^{\sim}{ }^{\sim}=1782(\mathrm{~m}), 1726(\mathrm{~s}, \mathrm{br}), 1499(\mathrm{~s}), 1391(\mathrm{~s}), 1219(\mathrm{~m}, \mathrm{C}-\mathrm{S})$, $1171(\mathrm{~s}), 1053$ (s, C=S) $\mathrm{cm}^{-1}$.

${ }^{1} \mathrm{H}-\mathrm{NMR}: \quad\left(400 \mathrm{MHz}, \mathrm{CDCl}_{3}\right): \delta=1.33-1.39(\mathrm{~m}, 12 \mathrm{H}), 1.43-1.47(\mathrm{~m}, 2 \mathrm{H}), 1.82-1.88$ (m, 2H), 1.90-2.15 (m, 2H), 2.75-2.85 (m, 1H), 2.92-3.04 (m, 1H), 3.68, 3.69 (each s, 3H), 4.08-4.15 (m, 1H), 4.27-4.38 (m, 1H), 4.52-4.60 (m, 2H), 5.17, 5.23 (each d, $\left.{ }^{3} \mathrm{~J}=8.0 \mathrm{~Hz}, 1 \mathrm{H}\right), 7.72-7.78(\mathrm{~m}, 2 \mathrm{H}), 7.83-7.87$ (m, $2 \mathrm{H})$.

${ }^{13} \mathrm{C}-\mathrm{NMR}: \quad\left(100.7 \mathrm{MHz}, \mathrm{CDCl}_{3}\right): \delta=13.57\left(\mathrm{CH}_{3}\right), 18.63,18.95,19.13\left(2 \times \mathrm{CH}_{2}\right), 28.15$ $\left(3 \times \mathrm{CH}_{3}\right), 35.06,35.83\left(\mathrm{CH}_{2}\right), 39.21\left(\mathrm{C}_{\mathrm{q}}\right), 41.46,42.99\left(\mathrm{CH}_{2}\right), 41.75,41.93$ $(\mathrm{CH}), 51.44,51.58\left(\mathrm{CH} / \mathrm{CH}_{3}\right), 52.32\left(\mathrm{CH} / \mathrm{CH}_{3}\right), 69.99\left(\mathrm{CH}_{2}\right), 79.85\left(\mathrm{C}_{\mathrm{q}}\right)$, $123.64(2 \times \mathrm{CH}), 131.32\left(2 \times \mathrm{C}_{\mathrm{q}}\right), 134.53(2 \times \mathrm{CH}), 155.11,155.35\left(\mathrm{C}_{\mathrm{q}}\right), 168.02$ $\left(2 \times \mathrm{C}_{\mathrm{q}}\right), 172.21,172.55\left(\mathrm{C}_{\mathrm{q}}\right), 203.32,203.83\left(\mathrm{C}_{\mathrm{q}}\right), 212.50,213.37\left(\mathrm{C}_{\mathrm{q}}\right)$.

ESI-MS: $\quad 582\left(\mathrm{M}^{+}+\mathrm{NH}_{4}\right), 565\left(\mathrm{M}^{+}+\mathrm{H}\right)$.

EI-HRMS: $\quad \mathrm{C}_{26} \mathrm{H}_{32} \mathrm{~N}_{2} \mathrm{O}_{8} \mathrm{~S}_{2}$, calcd.: 564.1600, found: 564.1607. $\mathrm{C}_{26} \mathrm{H}_{32} \mathrm{~N}_{2} \mathrm{O}_{8} \mathrm{~S}_{2}(564.67)$

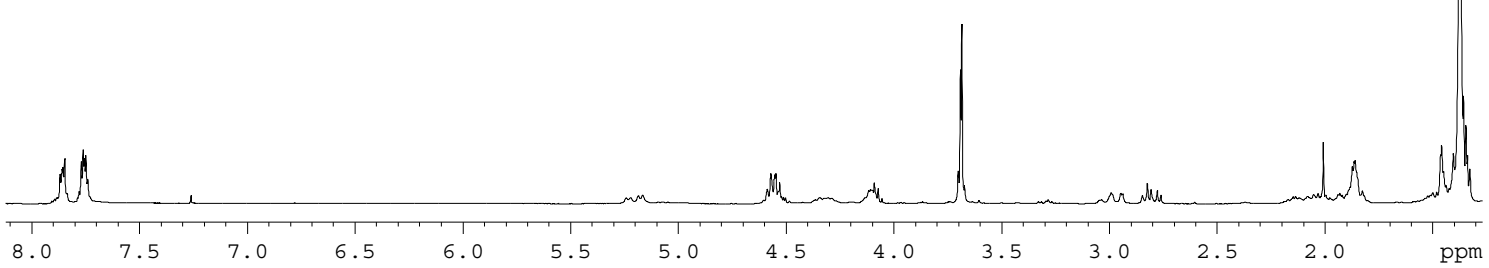




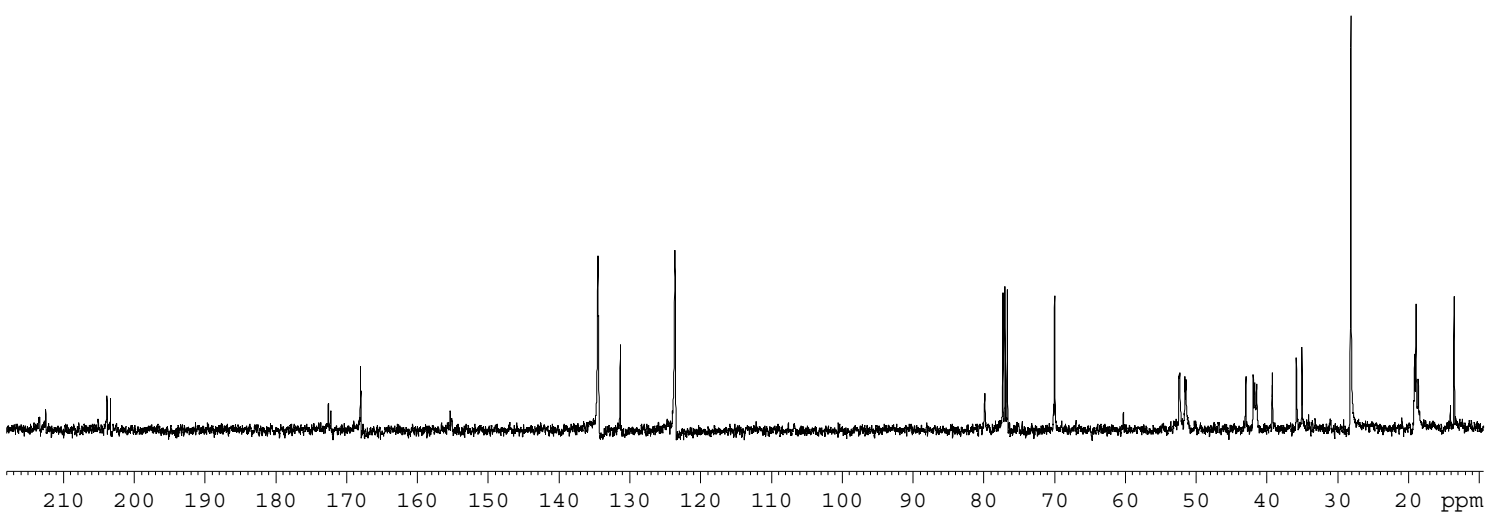

(E)-2-tert-Butoxycarbonylamino-6-[1-(1,3-dioxo-1,3-dihydro-isoindol-2-yl)-cyclopropyl]6-oxo-hex-4-enoic acid methyl ester (25)

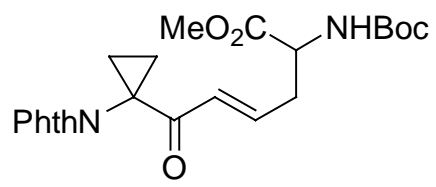

25

To a solution of $24(27 \mathrm{mg}, 0.048 \mathrm{mmol})$ in dichloromethane $(4 \mathrm{~mL})$ at $0{ }^{\circ} \mathrm{C}$ was added $\mathrm{DBU}$ (ca. $0.015 \mathrm{~mL}, 0.100 \mathrm{mmol}$ ). After $1 \mathrm{~h}$ at $0^{\circ} \mathrm{C}$ the mixture was washed with satd. ammonium chloride, dried over $\mathrm{Na}_{2} \mathrm{SO}_{4}$ and concentrated. Purification by column chromatography (3:7 EtOAc-hexanes) gave 25 (18 mg, $0.041 \mathrm{mmol}, 86 \%$ ).

$R_{\mathrm{f}}: \quad 0.30(3: 7$ EtOAc-hexanes $)$

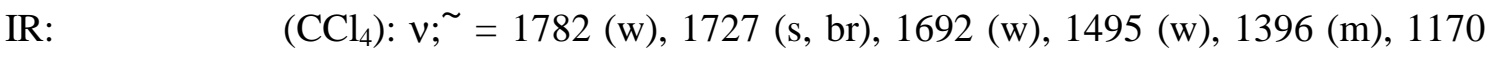
(w) $\mathrm{cm}^{-1}$.

${ }^{1} \mathrm{H}-\mathrm{NMR}: \quad\left(400 \mathrm{MHz}, \mathrm{CDCl}_{3}\right): \delta=1.41(\mathrm{~m}, 9 \mathrm{H}), 1.53-1.57(\mathrm{~m}, 2 \mathrm{H}), 1.87-1.92(\mathrm{~m}$, 2H), 2.55-2.62 (m, 1H), 2.64-2.72 (m, 1H), $3.71(\mathrm{~s}, 3 \mathrm{H}), 4.39-4.45(\mathrm{~m}$, $1 \mathrm{H}), 5.06\left(\mathrm{~d},{ }^{3} J=7.6 \mathrm{~Hz}, 1 \mathrm{H}\right), 6.14\left(\mathrm{~d},{ }^{3} J=15.2 \mathrm{~Hz}, 1 \mathrm{H}\right), 6.85\left(\mathrm{ddd},{ }^{3} J=\right.$ $\left.15.2 \mathrm{~Hz},{ }^{3} J=7.4 \mathrm{~Hz},{ }^{3} J=7.4 \mathrm{~Hz}, 1 \mathrm{H}\right), 7.75-7.79(\mathrm{~m}, 2 \mathrm{H}), 7.86-7.89$ (m, $2 \mathrm{H})$.

${ }^{13} \mathrm{C}-\mathrm{NMR}: \quad\left(100.7 \mathrm{MHz}, \mathrm{CDCl}_{3}\right): \delta=17.33\left(\mathrm{CH}_{2}\right), 17.38\left(\mathrm{CH}_{2}\right), 28.26\left(3 \times \mathrm{CH}_{3}\right), 35.28$ $\left(\mathrm{CH}_{2}\right), 39.29\left(\mathrm{C}_{\mathrm{q}}\right), 52.37(\mathrm{CH}), 52.51\left(\mathrm{CH}_{3}\right), 80.26\left(\mathrm{C}_{\mathrm{q}}\right), 123.60(2 \times \mathrm{CH})$, $124.60(\mathrm{CH}), 131.62\left(2 \times \mathrm{C}_{\mathrm{q}}\right), 134.37(2 \times \mathrm{CH}), 142.93(\mathrm{CH}), 154.99\left(\mathrm{C}_{\mathrm{q}}\right)$, $168.18\left(2 \times \mathrm{C}_{\mathrm{q}}\right), 171.70\left(\mathrm{C}_{\mathrm{q}}\right), 193.54\left(\mathrm{C}_{\mathrm{q}}\right)$.

ESI-MS: $\quad 460\left(\mathrm{M}^{+}+\mathrm{NH}_{4}\right), 443\left(\mathrm{M}^{+}+\mathrm{H}\right)$. 


$$
\mathrm{C}_{23} \mathrm{H}_{26} \mathrm{~N}_{2} \mathrm{O}_{7}(442.46)
$$

FAB-HRMS: $\quad \mathrm{C}_{23} \mathrm{H}_{26} \mathrm{~N}_{2} \mathrm{O}_{7} \mathrm{Na}^{+}$, calcd.: 465.1638, found: 465.1664 .

$\mathrm{C}_{23} \mathrm{H}_{26} \mathrm{~N}_{2} \mathrm{O}_{7}(442.46)$
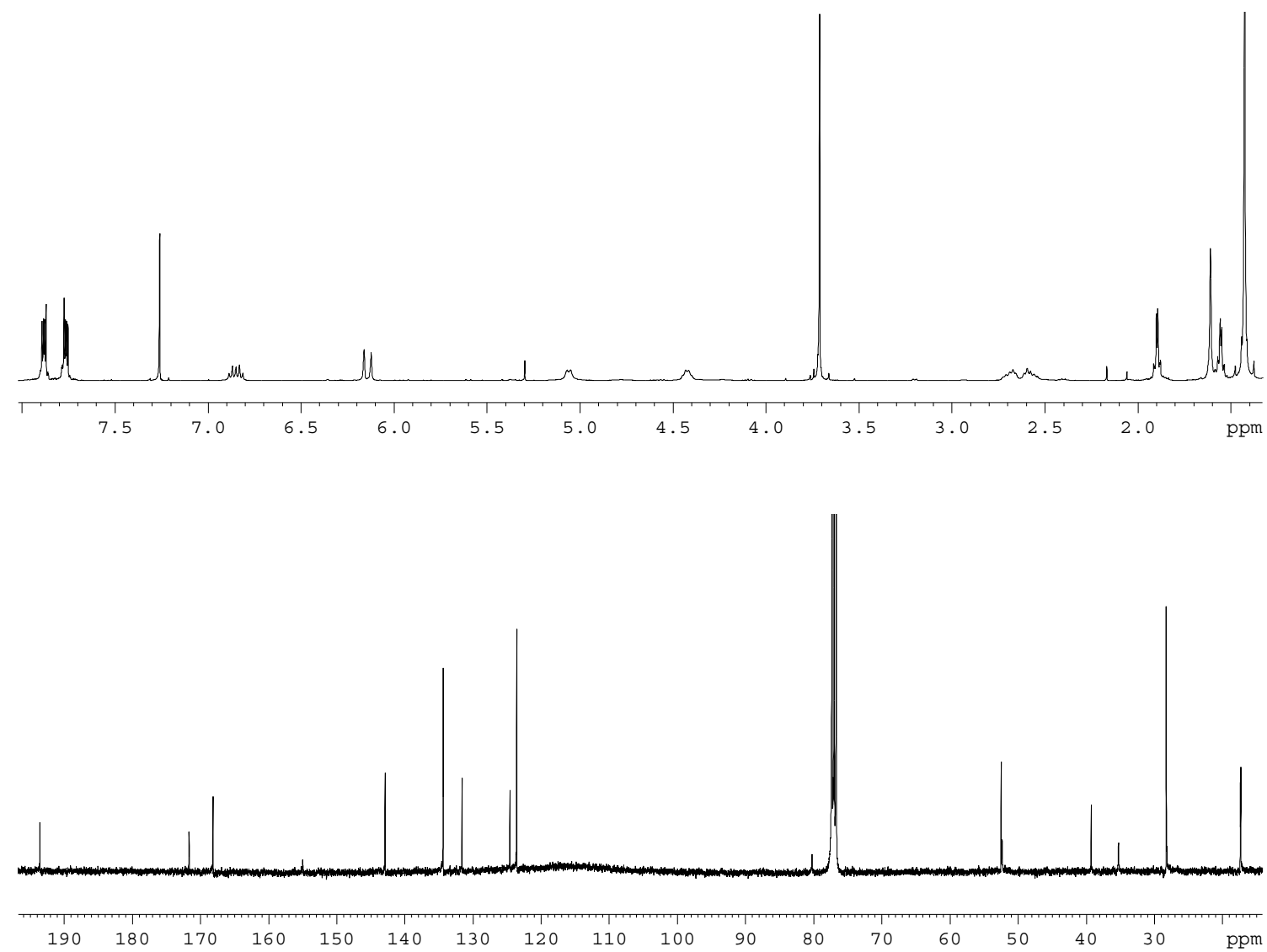

2-tert-Butoxycarbonylamino-6-[1-(1,3-dioxo-1,3-dihydro-isoindol-2-yl)-cyclopropyl]-6oxo-hex-2-enoic acid methyl ester (27)

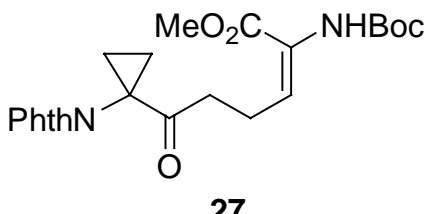

To a solution of $\mathbf{2 4}$ (31 $\mathrm{mg}, 0.055 \mathrm{mmol})$ in dichloromethane $(10 \mathrm{~mL})$ under argon was added $\mathrm{DBU}(0.017 \mathrm{~mL}, 0.110 \mathrm{mmol})$ and $\mathrm{Na}_{2} \mathrm{SO}_{4}(50 \mathrm{mg})$. After $48 \mathrm{~h}$ at r.t. the mixture was washed with satd. ammonium chloride, dried over $\mathrm{Na}_{2} \mathrm{SO}_{4}$ and concentrated. Purification by column chromatography (3:7 EtOAc-hexanes) gave a 4:1 mixture (22 $\mathrm{mg}, 0.050 \mathrm{mmol}, 90 \%)$ of 27 (72\%) and $25(18 \%)$. Only a small amount (5 mg) of 27 could be separated of 25. 
IR: $\left(\mathrm{CCl}_{4}\right): \mathrm{v} ; \sim=3436(\mathrm{w}), 1782(\mathrm{w}), 1727(\mathrm{~s}), 1492(\mathrm{~m}), 1395(\mathrm{~m}), 1169(\mathrm{~m})$ $\mathrm{cm}^{-1}$.

${ }^{1} \mathrm{H}-\mathrm{NMR}: \quad\left(400 \mathrm{MHz}, \mathrm{CDCl}_{3}\right): \delta=1.42-1.47(\mathrm{~m}, 2 \mathrm{H}), 1.45(\mathrm{~s}, 9 \mathrm{H}), 1.87-1.91(\mathrm{~m}, 2 \mathrm{H})$, $2.41\left(\mathrm{dt},{ }^{3} \mathrm{~J}=7.2 \mathrm{~Hz},{ }^{3} \mathrm{~J}=7.0 \mathrm{~Hz}, 2 \mathrm{H}\right), 2.60\left(\mathrm{t},{ }^{3} \mathrm{~J}=7.0 \mathrm{~Hz}, 2 \mathrm{H}\right), 3.75(\mathrm{~s}$, $3 \mathrm{H}), 6.29\left(\mathrm{t},{ }^{3} \mathrm{~J}=7.2 \mathrm{~Hz}, 1 \mathrm{H}\right), 6.33(\mathrm{~s}, 1 \mathrm{H}), 7.76-7.80(\mathrm{~m}, 2 \mathrm{H}), 7.87-7.91$ (m, 2H).

${ }^{13} \mathrm{C}-\mathrm{NMR}: \quad\left(100.7 \mathrm{MHz}, \mathrm{CDCl}_{3}\right): \delta=18.82\left(2 \times \mathrm{CH}_{2}\right), 22.31\left(\mathrm{CH}_{2}\right), 28.17\left(3 \times \mathrm{CH}_{3}\right)$, $36.20\left(\mathrm{CH}_{2}\right), 39.22\left(\mathrm{C}_{\mathrm{q}}\right), 52.23\left(\mathrm{CH}_{3}\right), 80.54\left(\mathrm{C}_{\mathrm{q}}\right), 123.69(2 \times \mathrm{CH}), 131.52$ $\left(2 \times \mathrm{C}_{\mathrm{q}}\right), 133.23(\mathrm{CH}), 134.52(2 \times \mathrm{CH}), 153.35\left(\mathrm{C}_{\mathrm{q}}\right), 168.18\left(2 \times \mathrm{C}_{\mathrm{q}}\right), 205.10$ $\left(\mathrm{C}_{\mathrm{q}}\right) \cdot\left(2 \times \mathrm{C}_{\mathrm{q}}\right.$ missing $)$

ESI-MS: $\quad 460\left(\mathrm{M}^{+}+\mathrm{NH}_{4}\right), 443\left(\mathrm{M}^{+}+\mathrm{H}\right)$.

FAB-HRMS: $\quad \mathrm{C}_{23} \mathrm{H}_{26} \mathrm{~N}_{2} \mathrm{O}_{7} \mathrm{Na}^{+}$, calcd.: 465.1638, found: 465.1651 .

$\mathrm{C}_{23} \mathrm{H}_{27} \mathrm{~N}_{2} \mathrm{O}_{7}^{+}$, calcd.: 443.1818, found: 443.1800.

$\mathrm{C}_{23} \mathrm{H}_{26} \mathrm{~N}_{2} \mathrm{O}_{7}(442.46)$
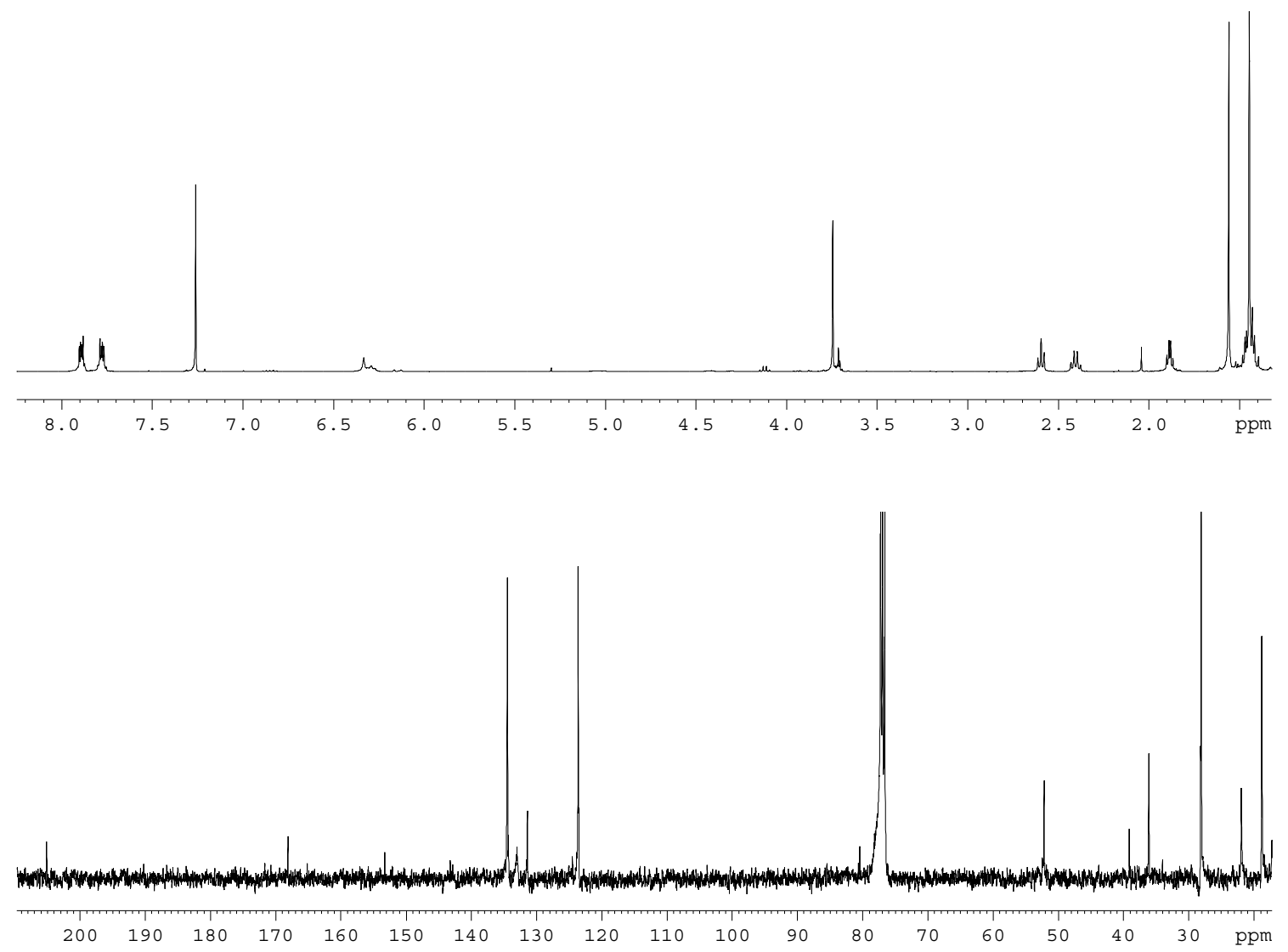
Dithiocarbonic acid (cyclopropylacyl) ester ethyl ester (28)<smiles>CCO[Se]C(=O)C1CC1</smiles>

28

To a solution of cyclopropanecarbonyl chloride $(1.60 \mathrm{~g}, 15.3 \mathrm{mmol})$ in acetone $(30 \mathrm{~mL})$ at $0^{\circ} \mathrm{C}$ was added ethyl xanthic acid potassium salt $(2.45 \mathrm{~g}, 15.3 \mathrm{mmol})$. After stirring for $15 \mathrm{~min}$ at $0^{\circ} \mathrm{C}$ the solvent was removed under reduced pressure at $0-10^{\circ} \mathrm{C}$. The residue was dissolved in dichloromethane, followed by water and brine and dried over $\mathrm{Na}_{2} \mathrm{SO}_{4}$. Evaporation of the solvent gave acyl xanthate $\mathbf{2 8}$ (2.71 g, $14.2 \mathrm{mmol}, 93 \%)$

$R_{\mathrm{f}}: \quad 0.85(1: 10$ EtOAc-hexanes $)$

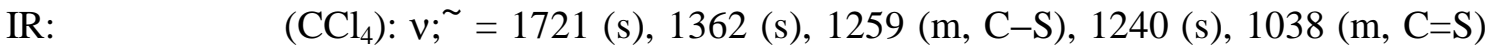
$\mathrm{cm}^{-1}$.

${ }^{1} \mathrm{H}-\mathrm{NMR}: \quad\left(400 \mathrm{MHz}, \mathrm{CDCl}_{3}\right): \delta=1.03-1.08(\mathrm{~m}, 2 \mathrm{H}), 1.23-1.26(\mathrm{~m}, 2 \mathrm{H}), 1.46\left(\mathrm{t},{ }^{3} \mathrm{~J}=\right.$ $7.2 \mathrm{~Hz}, 3 \mathrm{H}), 1.92-1.99(\mathrm{~m}, 1 \mathrm{H}), 4.66\left(\mathrm{q},{ }^{3} \mathrm{~J}=7.2 \mathrm{~Hz}, 2 \mathrm{H}\right)$.

${ }^{13} \mathrm{C}-\mathrm{NMR}: \quad\left(100.7 \mathrm{MHz}, \mathrm{CDCl}_{3}\right): \delta=12.01\left(2 \times \mathrm{CH}_{2}\right), 13.43\left(\mathrm{CH}_{3}\right), 22.39(\mathrm{CH}), 70.80$ $\left(\mathrm{CH}_{2}\right), 192.33\left(\mathrm{C}_{\mathrm{q}}\right), 203.89\left(\mathrm{C}_{\mathrm{q}}\right)$.

ESI-MS: $\quad 208\left(\mathrm{M}^{+}+\mathrm{NH}_{4}\right), 191\left(\mathrm{M}^{+}+\mathrm{H}\right)$

EI-HRMS: $\quad \mathrm{C}_{7} \mathrm{H}_{10} \mathrm{O}_{2} \mathrm{~S}_{2}$, calcd.: 190.0122, found: 190.0118 .

$\mathrm{C}_{7} \mathrm{H}_{10} \mathrm{O}_{2} \mathrm{~S}_{2}(190.29)$

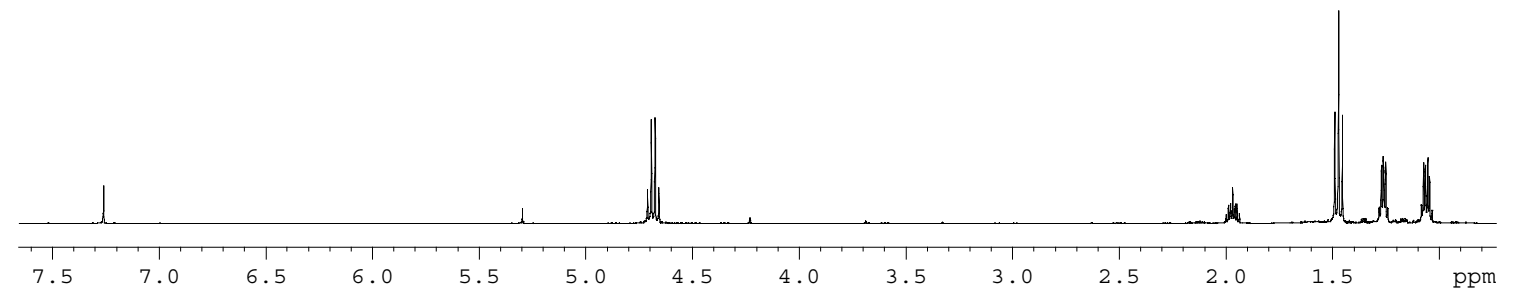




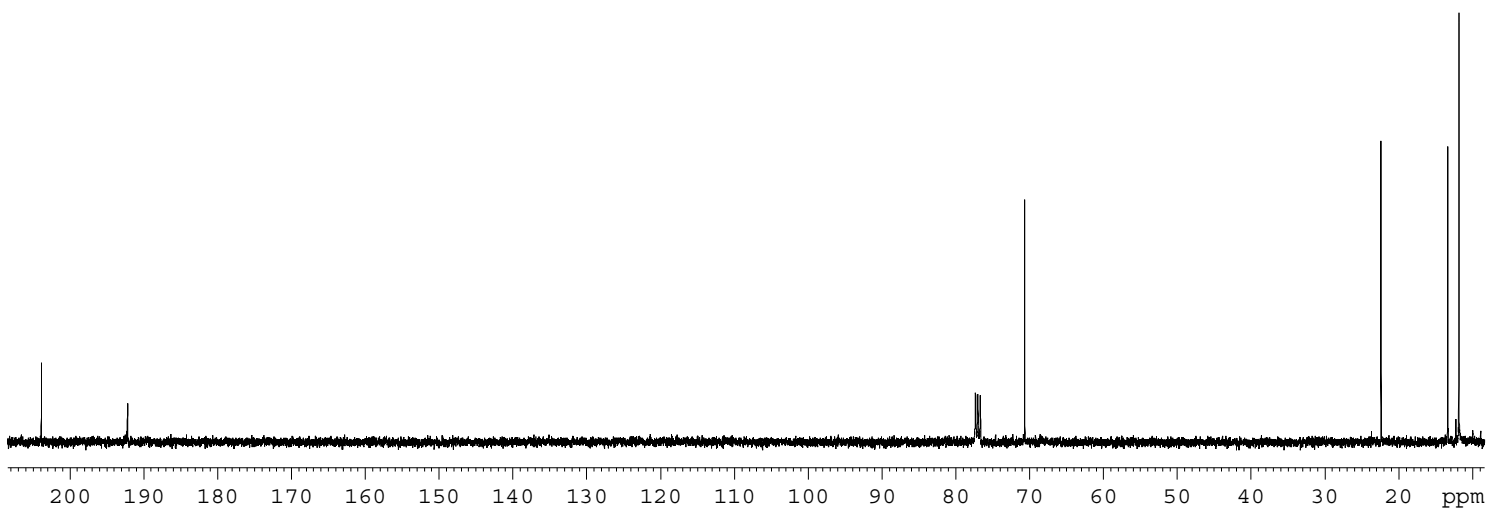

(E)-1-Cyclopropyl-non-2-en-1-one (30) ${ }^{4}$

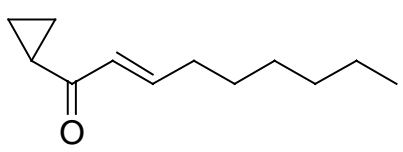

30

A solution of xanthate $28(141 \mathrm{mg}, 0.74 \mathrm{mmol})$ in 1-octene $(1.50 \mathrm{~mL})$ was exposed to visible light and refluxed for $2 \mathrm{~h}$. The solvent was removed under reduced pressure and the residue was dissolved in dichloromethane $(5 \mathrm{~mL})$. After the addition of DBU $(0.14 \mathrm{~mL}, 140 \mathrm{mg}, 0.92$ $\mathrm{mmol}$ ) the reaction mixture was stirred for $40 \mathrm{~min}$ at r.t. and finally quenched with satd. ammonium chloride $(10 \mathrm{~mL})$. Extraction with dichloromethane, drying over $\mathrm{Na}_{2} \mathrm{SO}_{4}$ and purification by column chromatography (3:97 EtOAc-hexanes) gave $\mathbf{3 0}$ (106 mg, $0.59 \mathrm{mmol}$, $79 \%)$.

$R_{\mathrm{f}}: \quad 0.30(3: 97$ EtOAc- hexanes $)$

IR: $\quad\left(\mathrm{CCl}_{4}\right): \mathrm{v}^{\sim}=2958(\mathrm{~m}), 2929(\mathrm{~s}), 2856(\mathrm{~m}), 1686(\mathrm{~s}), 1666(\mathrm{~s}), 1627(\mathrm{~s})$, $1387(\mathrm{~s}) \mathrm{cm}^{-1}$.

${ }^{1} \mathrm{H}-\mathrm{NMR}: \quad\left(400 \mathrm{MHz}, \mathrm{CDCl}_{3}\right): \delta=0.85-0.91(\mathrm{~m}, 5 \mathrm{H}), 1.04-1.08(\mathrm{~m}, 2 \mathrm{H}), 1.24-1.35$ $(\mathrm{m}, 6 \mathrm{H}), 1.47\left(\mathrm{tt},{ }^{3} J=7.0 \mathrm{~Hz},{ }^{3} J=7.0 \mathrm{~Hz}, 2 \mathrm{H}\right), 2.09-2.16(\mathrm{~m}, 1 \mathrm{H}), 2.22$ (tdd, $\left.{ }^{3} J=7.0 \mathrm{~Hz},{ }^{3} J=6.8 \mathrm{~Hz},{ }^{4} J=1.4 \mathrm{~Hz}, 2 \mathrm{H}\right), 6.21\left(\mathrm{dt},{ }^{3} \mathrm{~J}=15.6 \mathrm{~Hz},{ }^{4} \mathrm{~J}=\right.$ $1.4 \mathrm{~Hz}, 1 \mathrm{H}), 6.90\left(\mathrm{dt},{ }^{3} J=15.6 \mathrm{~Hz},{ }^{3} J=6.8 \mathrm{~Hz}, 1 \mathrm{H}\right)$.

${ }^{13} \mathrm{C}-\mathrm{NMR}: \quad\left(100.7 \mathrm{MHz}, \mathrm{CDCl}_{3}\right): \delta=10.93\left(2 \times \mathrm{CH}_{2}\right), 14.01\left(\mathrm{CH} / \mathrm{CH}_{3}\right), 18.56$ $\left(\mathrm{CH} / \mathrm{CH}_{3}\right), 22.49\left(\mathrm{CH}_{2}\right), 28.06\left(\mathrm{CH}_{2}\right), 28.82\left(\mathrm{CH}_{2}\right), 31.55\left(\mathrm{CH}_{2}\right), 32.46$ $\left(\mathrm{CH}_{2}\right), 130.36(\mathrm{CH}), 147.10(\mathrm{CH}), 200.36\left(\mathrm{C}_{\mathrm{q}}\right)$.

ESI-MS: $\quad 198\left(\mathrm{M}^{+}+\mathrm{NH}_{4}\right), 181\left(\mathrm{M}^{+}+\mathrm{H}\right)$.

EI-HRMS: $\quad \mathrm{C}_{12} \mathrm{H}_{20} \mathrm{O}$, calcd.: 180.1514 , found: 180.1527. 


$$
\mathrm{C}_{12} \mathrm{H}_{20} \mathrm{O}(180.29)
$$
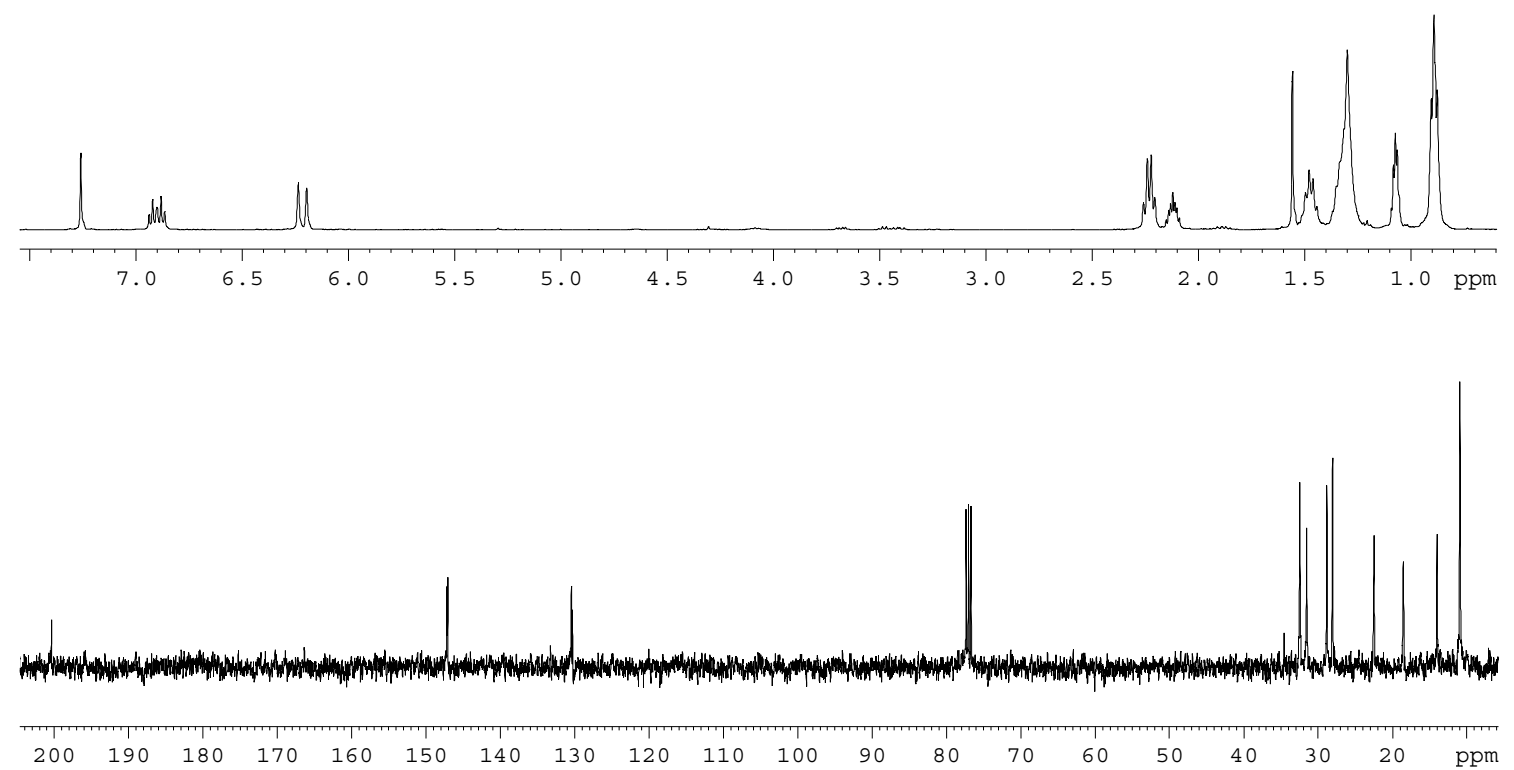

\section{2,2-Dimethyl-propionic acid 3-cyclopropyl-1-ethoxythiocarbonylsulfanyl-3-oxo-propyl} ester (31)

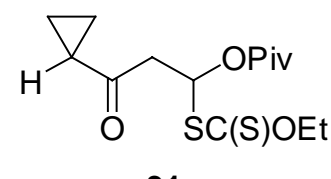

31

A solution of xanthate $28(170 \mathrm{mg}, 0.89 \mathrm{mmol})$ in vinyl pivalate $(1.50 \mathrm{~mL})$ was exposed to visible light and refluxed for $7.5 \mathrm{~h}$. The solvent was removed under reduced pressure and the residue was purified by column chromatography (1:20 EtOAc- hexanes and $\left.100 \% \mathrm{CH}_{2} \mathrm{Cl}_{2}\right)$ to give 31 (102 mg, $0.16 \mathrm{mmol}, 36 \%$, calculated from NMR spectra) along with minor amounts of cyclopropane carboxylic acid. Due to difficulties with purification and decomposition 31 was directly converted to $\mathbf{3 2}$.

$R_{\mathrm{f}}: \quad 0.25(1: 20$ EtOAc-hexanes $)$

${ }^{1} \mathrm{H}-\mathrm{NMR}: \quad\left(400 \mathrm{MHz}, \mathrm{CDCl}_{3}\right): \delta=0.91-0.96(\mathrm{~m}, 2 \mathrm{H}), 1.03-1.09(\mathrm{~m}, 2 \mathrm{H}), 1.17(\mathrm{~s}, 9 \mathrm{H})$, $1.43\left(\mathrm{t},{ }^{3} J=7.0 \mathrm{~Hz}, 3 \mathrm{H}\right), 1.93-2.00(\mathrm{~m}, 1 \mathrm{H}), 3.22\left(\mathrm{dd},{ }^{2} J=16.4 \mathrm{~Hz},{ }^{3} J=7.0\right.$ $\mathrm{Hz}, 1 \mathrm{H}), 3.27\left(\mathrm{dd},{ }^{2} J=16.4 \mathrm{~Hz},{ }^{3} J=5.8 \mathrm{~Hz}, 1 \mathrm{H}\right), 4.65\left(\mathrm{t},{ }^{3} J=7.0 \mathrm{~Hz}, 2 \mathrm{H}\right)$, $6.92\left(\mathrm{dd},{ }^{3} \mathrm{~J}=7.0 \mathrm{~Hz},{ }^{3} \mathrm{~J}=5.8 \mathrm{~Hz}, 1 \mathrm{H}\right)$. 
${ }^{13} \mathrm{C}-\mathrm{NMR}: \quad\left(100.7 \mathrm{MHz}, \mathrm{CDCl}_{3}\right): \delta=11.38\left(2 \times \mathrm{CH}_{2}\right), 13.62\left(\mathrm{CH}_{3}\right), 20.75(\mathrm{CH}), 26.81$ $\left(3 \times \mathrm{CH}_{3}\right), 38.79\left(\mathrm{C}_{\mathrm{q}}\right), 47.30\left(\mathrm{CH}_{2}\right), 70.16\left(\mathrm{CH}_{2}\right), 76.27(\mathrm{CH}), 176.42\left(\mathrm{C}_{\mathrm{q}}\right)$, $205.09\left(\mathrm{C}_{\mathrm{q}}\right), 210.20\left(\mathrm{C}_{\mathrm{q}}\right)$.

ESI-MS: $\quad 336\left(\mathrm{M}^{+}+\mathrm{NH}_{4}\right), 319\left(\mathrm{M}^{+}+\mathrm{H}\right)$.

$\mathrm{C}_{14} \mathrm{H}_{22} \mathrm{O}_{4} \mathrm{~S}_{2}(318.45)$

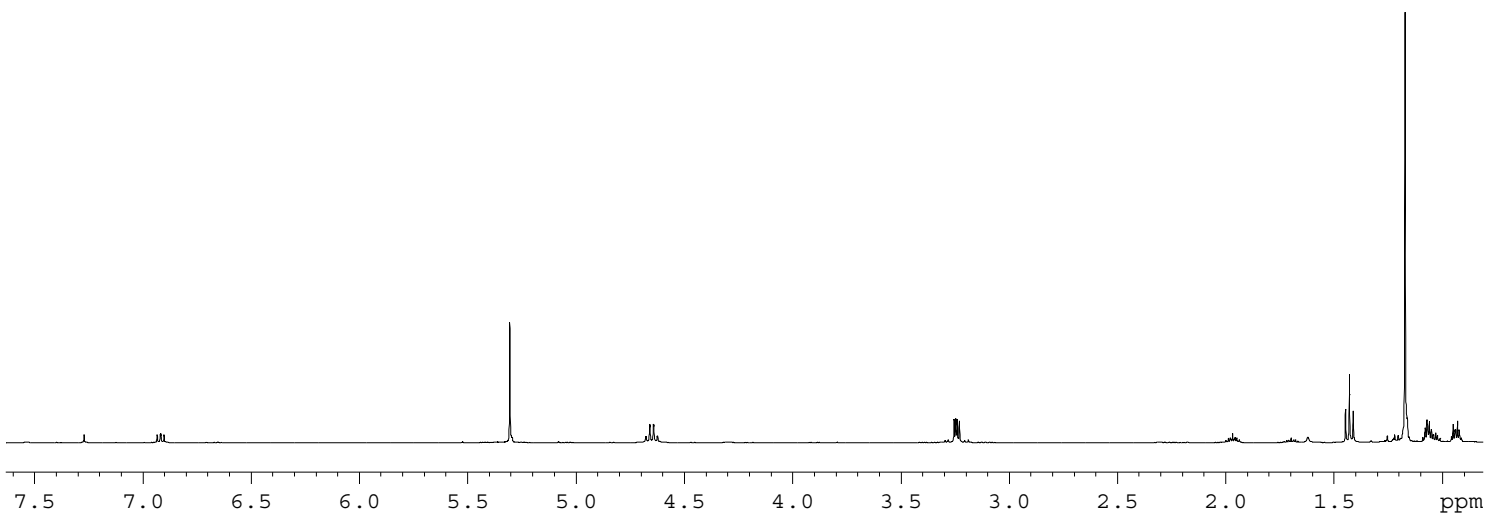

3-Cyclopropyl-1H-pyrazole (32)

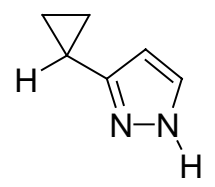

32

To a solution of crude $31(\sim 0.23 \mathrm{mmol})$ in acetic acid $(1 \mathrm{~mL})$ was added hydrazine hydrate $(0.13 \mathrm{~mL}, 2.5 \mathrm{mmol})$. The resulting mixture was stirred for $45 \mathrm{~min}$ at r.t. and diluted with $2 \mathrm{~N}$ $\mathrm{HCl}(10 \mathrm{~mL})$. After washing with dichloromethane $(2 \times 10 \mathrm{~mL})$, the aqueous phase was basified with satd. $\mathrm{Na}_{2} \mathrm{CO}_{3}$. Extraction with dichloromethane $(2 \times 10 \mathrm{~mL})$, drying over $\mathrm{Na}_{2} \mathrm{SO}_{4}$, purification by column chromatography (1:4 EtOAc-hexanes to $100 \%$ EtOAc) and sublimation under atmospheric pressure gave $32(15 \mathrm{mg}, 0.14 \mathrm{mmol}, 60 \%)$.

$R_{\mathrm{f}}: \quad 0.85(100 \%$ EtOAc $)$

IR: $\quad\left(\mathrm{CCl}_{4}\right): \mathrm{v}^{\sim} \quad 3481(\mathrm{~s}), 3193(\mathrm{~s}, \mathrm{br}), 3007(\mathrm{~s}, \mathrm{br}), 1534(\mathrm{~m}), 1499(\mathrm{~m}), 1470$ (m), 1369 (m), 1264 (m), 1240 (m), 1104 (m), 1026 (m) cm $\mathrm{cm}^{-1}$.

${ }^{1} \mathrm{H}-\mathrm{NMR}: \quad\left(400 \mathrm{MHz}, \mathrm{CDCl}_{3}\right): \delta=0.73(\mathrm{ddd}, J=6.8 \mathrm{~Hz}, J=6.4 \mathrm{~Hz}, J=4.4 \mathrm{~Hz}, 2 \mathrm{H})$, $0.94(\mathrm{ddd}, J=8.4 \mathrm{~Hz}, J=6.4 \mathrm{~Hz}, J=4.4 \mathrm{~Hz}, 2 \mathrm{H}), 1.90-1.96(\mathrm{~m}, 1 \mathrm{H}), 5.96$ $\left(\mathrm{d},{ }^{3} \mathrm{~J}=2.0 \mathrm{~Hz}, 1 \mathrm{H}\right), 7.45\left(\mathrm{~d},{ }^{3} \mathrm{~J}=2.0 \mathrm{~Hz}, 1 \mathrm{H}\right)$. 
${ }^{13} \mathrm{C}-\mathrm{NMR}: \quad\left(100.7 \mathrm{MHz}, \mathrm{CDCl}_{3}\right): \delta=7.68\left(2 \times \mathrm{CH}_{2}\right), 7.82(\mathrm{CH}), 101.59(\mathrm{CH}), 134.10$ $(\mathrm{CH}), 150.99\left(\mathrm{C}_{\mathrm{q}}\right)$.

ESI-MS: $\quad 109\left(\mathrm{M}^{+}+\mathrm{H}\right)$.

EI-HRMS: $\quad \mathrm{C}_{6} \mathrm{H}_{8} \mathrm{~N}_{2}$, calcd.: 108.0688, found: 108.0687 .

$\mathrm{C}_{6} \mathrm{H}_{8} \mathrm{~N}_{2}(108.14)$
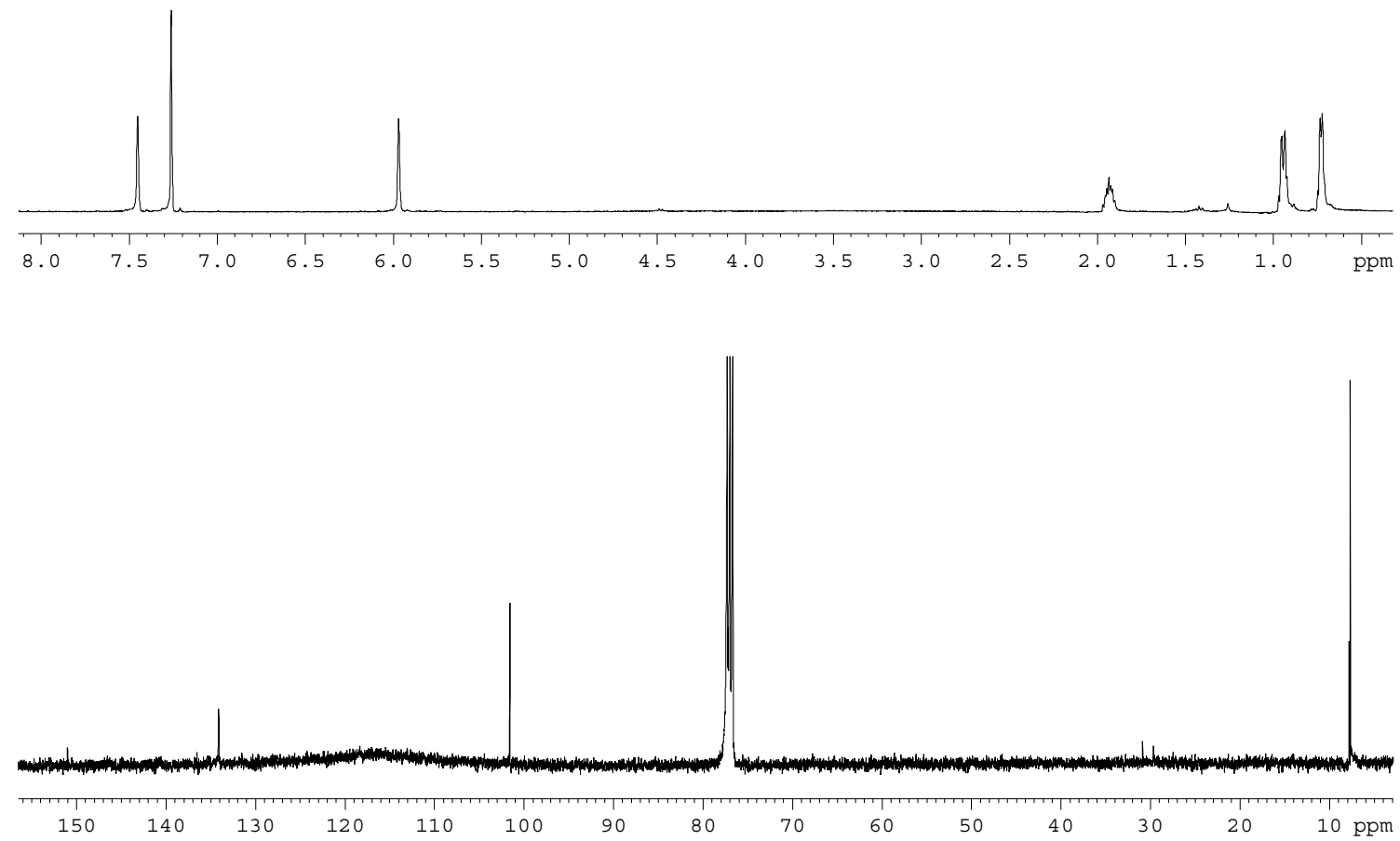

(1S,3R)-2,2-Dimethyl-3-[2-(2-methyl-[1,3]dithiolan-2-yl)-ethyl]-cyclopropanecarboxylic acid (precursor for 33)

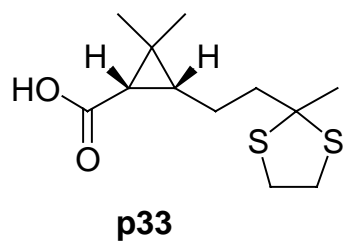

p33 was prepared according to ref. $^{5}$ from (1S,3R)-2,2-dimethyl-3-(3-oxo-butyl)cyclopropanecarboxylic $\operatorname{acid}^{6}(1.37 \mathrm{~g}, 7.42 \mathrm{mmol})$, 1,2-ethanedithiol $(0.62 \mathrm{~mL}, 0.70 \mathrm{~g}, 7.42$ $\mathrm{mmol}$ ) and boron trifluoride etherate $(1.10 \mathrm{~mL}, 1.26 \mathrm{~g}, 8.90 \mathrm{mmol})$. Purification by column chromatography gave p33 (1.70 g, $6.53 \mathrm{mmol}, 88 \%)$.

$R_{\mathrm{f}}: \quad 0.35(1: 10$ EtOAc-hexanes $)$

m.p.: $\quad 97^{\circ} \mathrm{C}$ 
$\alpha_{\mathrm{D}}^{23}: \quad+20^{\circ}(\mathrm{c}=0.76$ in $\mathrm{MeOH})$

IR: $\quad\left(\mathrm{CCl}_{4}\right): \mathrm{v}^{\sim} \sim 2958(\mathrm{~m}), 2924(\mathrm{~m}), 1695(\mathrm{~s}), 1438(\mathrm{~m}), 1225(\mathrm{~s}) \mathrm{cm}^{-1}$.

${ }^{1} \mathrm{H}-\mathrm{NMR}: \quad\left(400 \mathrm{MHz}, \mathrm{CDCl}_{3}\right): \delta=1.13(\mathrm{~s}, 3 \mathrm{H}), 1.13-1.18(\mathrm{~m}, 1 \mathrm{H}), 1.21(\mathrm{~s}, 3 \mathrm{H}), 1.40$

$\left(\mathrm{d},{ }^{3} \mathrm{~J}=8.8 \mathrm{~Hz}, 1 \mathrm{H}\right), 1.71(\mathrm{~s}, 3 \mathrm{H}), 1.82-1.88(\mathrm{~m}, 2 \mathrm{H}), 1.90-1.94(\mathrm{~m}, 2 \mathrm{H})$, $3.23-3.35(\mathrm{~m}, 4 \mathrm{H})$.

${ }^{13} \mathrm{C}-\mathrm{NMR}: \quad\left(100.7 \mathrm{MHz}, \mathrm{CDCl}_{3}\right): \delta=14.25\left(\mathrm{CH}_{3}\right), 21.38\left(\mathrm{CH}_{2}\right), 26.86\left(\mathrm{C}_{\mathrm{q}}\right), 28.45$ $\left(\mathrm{CH} / \mathrm{CH}_{3}\right), 29.12\left(\mathrm{CH} / \mathrm{CH}_{3}\right), 31.84\left(\mathrm{CH}_{3}\right), 34.65\left(\mathrm{CH} / \mathrm{CH}_{3}\right), 39.69\left(\mathrm{CH}_{2}\right)$, $39.78\left(\mathrm{CH}_{2}\right), 45.65\left(\mathrm{CH}_{2}\right), 66.50\left(\mathrm{C}_{\mathrm{q}}\right), 178.47\left(\mathrm{C}_{\mathrm{q}}\right)$.

ESI-MS: $\quad 278\left(\mathrm{M}^{+}+\mathrm{NH}_{4}\right), 261\left(\mathrm{M}^{+}+\mathrm{H}\right), 243\left(\mathrm{M}^{+}-\mathrm{OH}\right)$.

$\mathrm{C}_{12} \mathrm{H}_{20} \mathrm{O}_{2} \mathrm{~S}_{2}(260.42)$

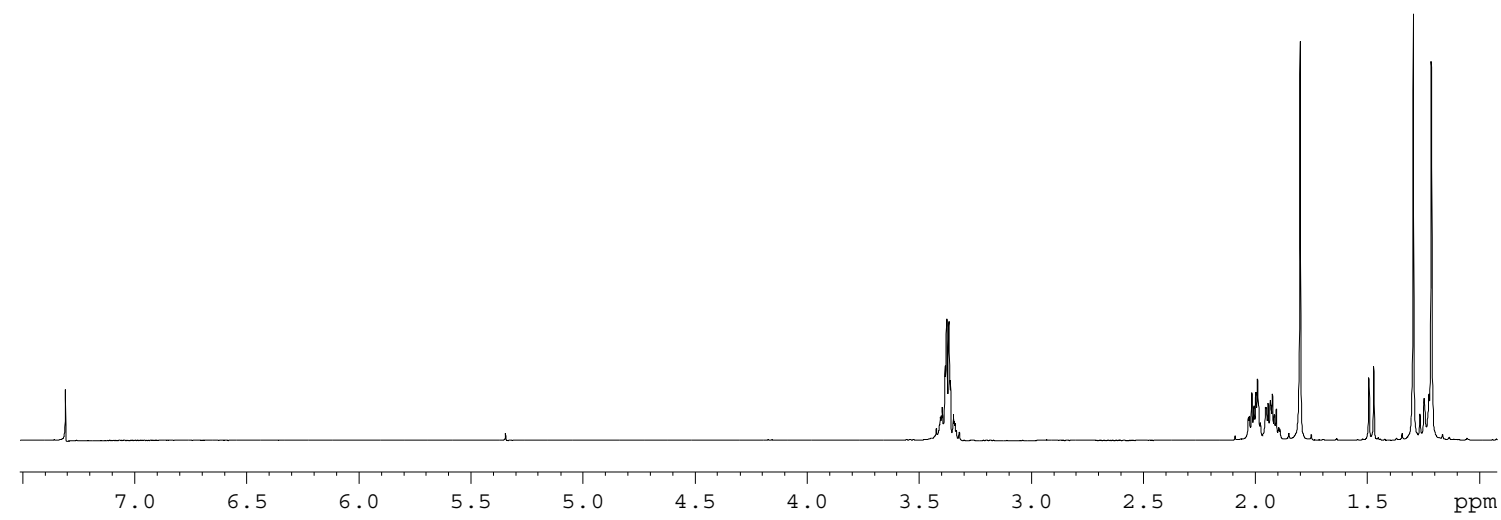

Dithiocarbonic acid [(1S,3R)-2,2-dimethyl-3-(3-oxo-butyl)-cyclopropylacyl] ester ethyl ester (33)

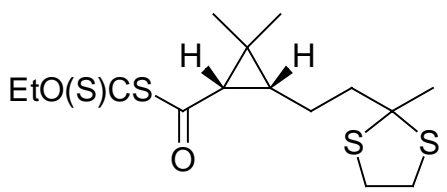

33

To an ice-cooled solution of acid p33 $(0.60 \mathrm{~g}, 2.30 \mathrm{mmol})$ in dichloromethane $(10 \mathrm{~mL})$ under argon were added oxalyl chloride $(0.30 \mathrm{~mL}, 0.44 \mathrm{~g}, 3.46 \mathrm{mmol})$ and a catalytic amount of DMF. After stirring for $90 \mathrm{~min}$ at $0^{\circ} \mathrm{C}$, the solvent was removed under reduced pressure and the residue dried in vacuo. The crude acid chloride was dissolved in acetone $(20 \mathrm{~mL})$ at $0^{\circ} \mathrm{C}$ and ethyl xanthic acid potassium salt (0.36 g, $2.25 \mathrm{mmol})$ was added. After stirring for $15 \mathrm{~min}$ at $0^{\circ} \mathrm{C}$ the solvent was removed under reduced pressure at $0-10^{\circ} \mathrm{C}$. The residue was dissolved 
in hexanes, washed with water, followed by brine and dried over $\mathrm{Na}_{2} \mathrm{SO}_{4}$. Evaporation of the solvent gave acyl xanthate $\mathbf{3 3}(0.78 \mathrm{~g}, 2.15 \mathrm{mmol}, 93 \%)$

$R_{\mathrm{f}}: \quad 0.75(1: 10$ EtOAc-hexanes $)$

$\alpha_{\mathrm{D}}: \quad+40^{\circ}\left(\mathrm{c}=0.25\right.$ in $\left.\mathrm{CHCl}_{3}\right)$

IR: $\quad\left(\mathrm{CCl}_{4}\right): \mathrm{v}^{\sim}=2958(\mathrm{~m}), 2924(\mathrm{~m}), 1725(\mathrm{~s}), 1255(\mathrm{~s}, \mathrm{C}-\mathrm{S}), 1236(\mathrm{~s}), 1033$ (s, C=S), $983(\mathrm{~s}) \mathrm{cm}^{-1}$.

${ }^{1} \mathrm{H}-\mathrm{NMR}: \quad\left(400 \mathrm{MHz}, \mathrm{CDCl}_{3}\right): \delta=1.13(\mathrm{~s}, 3 \mathrm{H}), 1.22(\mathrm{~s}, 3 \mathrm{H}), 1.39-1.45(\mathrm{~m}, 1 \mathrm{H}), 1.43$ $\left(\mathrm{t},{ }^{3} J=7.0 \mathrm{~Hz}, 3 \mathrm{H}\right), 1.69\left(\mathrm{~d},{ }^{3} J=8.8 \mathrm{~Hz}, 1 \mathrm{H}\right), 1.71(\mathrm{~s}, 3 \mathrm{H}), 1.82-1.95(\mathrm{~m}$, $4 \mathrm{H}), 3.23-3.34(\mathrm{~m}, 4 \mathrm{H}), 4.64\left(\mathrm{q},{ }^{3} J=7.0 \mathrm{~Hz}, 2 \mathrm{H}\right)$.

${ }^{13} \mathrm{C}-\mathrm{NMR}: \quad\left(100.7 \mathrm{MHz}, \mathrm{CDCl}_{3}\right): \delta=13.46\left(\mathrm{CH}_{3}\right), 14.20\left(\mathrm{CH}_{3}\right), 21.42\left(\mathrm{CH}_{2}\right), 28.70$ $\left(\mathrm{CH}_{3}\right), 31.68\left(\mathrm{C}_{\mathrm{q}}\right), 31.81\left(\mathrm{CH}_{3}\right), 38.33(\mathrm{CH}), 39.02(\mathrm{CH}), 39.65\left(\mathrm{CH}_{2}\right), 39.74$ $\left(\mathrm{CH}_{2}\right), 45.35\left(\mathrm{CH}_{2}\right), 66.20\left(\mathrm{C}_{\mathrm{q}}\right), 70.36\left(\mathrm{CH}_{2}\right), 187.56\left(\mathrm{C}_{\mathrm{q}}\right), 205.14\left(\mathrm{C}_{\mathrm{q}}\right)$.

ESI-MS: $\quad 381\left(\mathrm{M}^{+}+\mathrm{NH}_{3}, 1 \%\right), 364\left(\mathrm{M}^{+}, 1 \%\right), 260\left(\mathrm{M}^{+}+\mathrm{NH}_{3}-\mathrm{C}_{3} \mathrm{H}_{5} \mathrm{OS}_{2}\right), 243$ $\left(\mathrm{M}^{+}-\mathrm{C}_{3} \mathrm{H}_{5} \mathrm{OS}_{2}\right)$.

$\mathrm{C}_{15} \mathrm{H}_{24} \mathrm{O}_{2} \mathrm{~S}_{4}(364.61)$
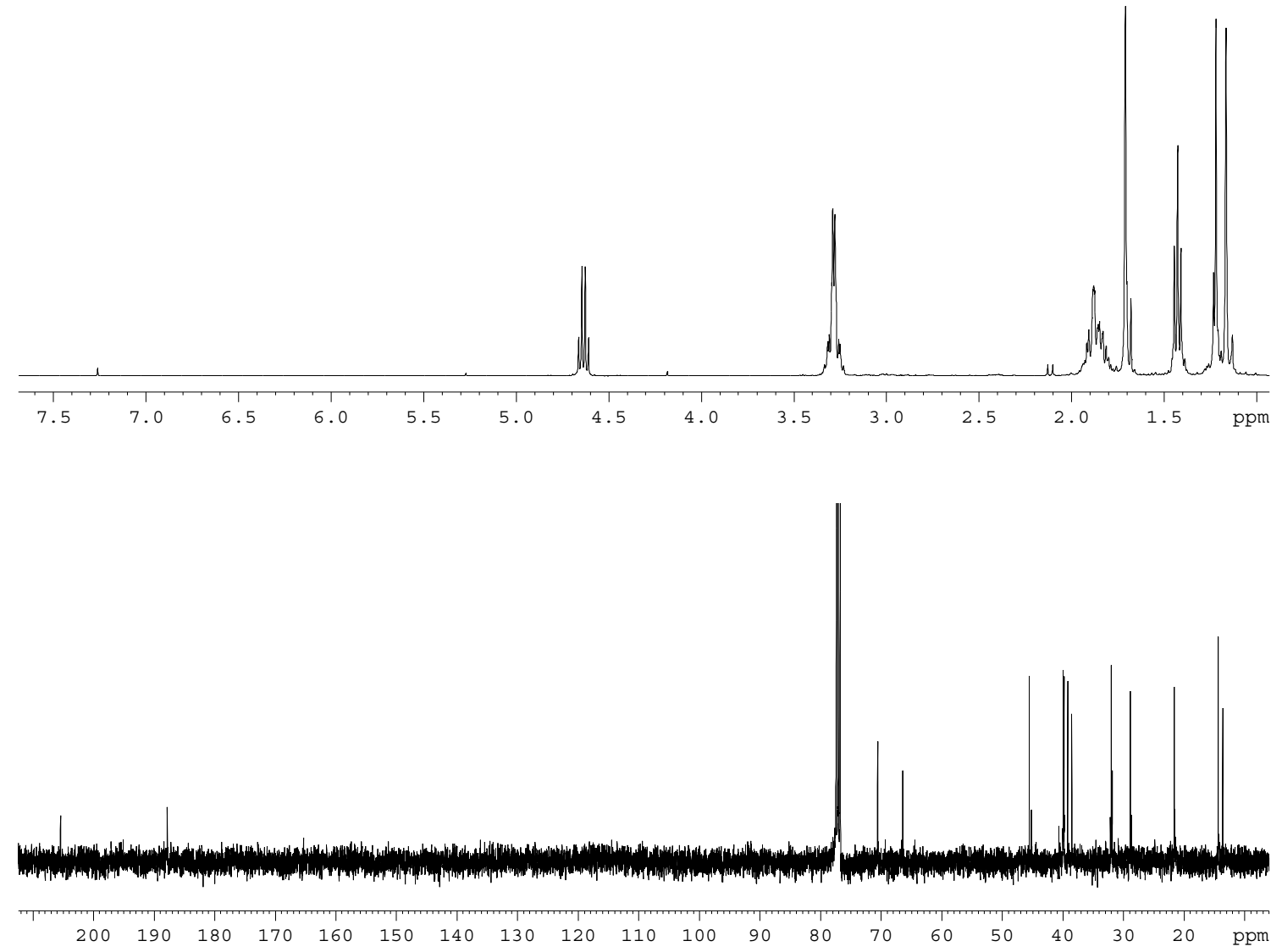
Dithiocarbonic acid (3-\{(1S,3R)-2,2-dimethyl-3-[2-(2-methyl-[1,3]dithiolan-2-yl)-ethyl]cyclopropyl\}-3-oxo-1-trimethylsilanyl-propyl) ester ethyl ester (34), mixture of diastereoisomers

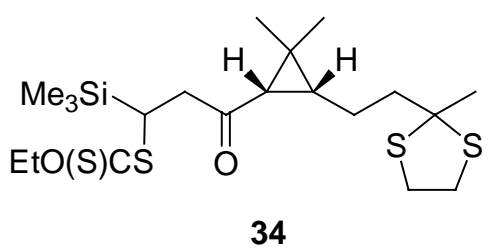

A solution of xanthate $33(212 \mathrm{mg}, 0.58 \mathrm{mmol})$ in vinyl trimethylsilane $(1.5 \mathrm{~mL})$ was exposed to visible light and refluxed for $8 \mathrm{~h}$ under argon. Concentration and purification by column chromatography (1:20 EtOAc-hexanes) gave 34 (113 mg, $0.24 \mathrm{mmol}, 42 \%)$ along with with ca. $10 \%$ of starting material $\mathbf{3 3}$.

$R_{\mathrm{f}}: \quad 0.65(1: 20$ EtOAc-hexanes $)$

IR: $\quad\left(\mathrm{CCl}_{4}\right): v^{\sim}{ }^{\sim}=2958(\mathrm{~m}), 2924(\mathrm{~m}), 1694(\mathrm{~m}), 1219(\mathrm{~s}, \mathrm{C}-\mathrm{S}), 1048(\mathrm{~s}, \mathrm{C}=\mathrm{S})$ $\mathrm{cm}^{-1}$.

${ }^{1} \mathrm{H}-\mathrm{NMR}: \quad\left(400 \mathrm{MHz}, \mathrm{CDCl}_{3}\right): \delta=0.08,0.09($ each s, $9 \mathrm{H}), 1.12-1.26(\mathrm{~m}, 7 \mathrm{H}), 1.40(\mathrm{t}$, $\left.{ }^{3} J=7.2 \mathrm{~Hz}, 3 \mathrm{H}\right), 1.70-1.76(\mathrm{~m}, 4 \mathrm{H}), 1.81-1.94(\mathrm{~m}, 4 \mathrm{H}), 2.84-3.00(\mathrm{~m}, 2 \mathrm{H})$, 3.26-3.36 (m, 4H), 3.49-3.58 (m, 1H), 4.58-4.67 (m, 2H).

${ }^{13} \mathrm{C}-\mathrm{NMR}: \quad\left(100.7 \mathrm{MHz}, \mathrm{CDCl}_{3}\right): \delta=-2.23,-2.20\left(3 \times \mathrm{CH}_{3}\right), 13.80\left(2 \times \mathrm{CH}_{3}\right), 20.98$, $21.01\left(\mathrm{CH}_{2}\right), 21.03\left(\mathrm{CH}_{2}\right), 29.40,29.42\left(\mathrm{CH}_{3}\right), 29.62,29.67\left(\mathrm{C}_{\mathrm{q}}\right)$, 31.93, $31.97\left(\mathrm{CH}_{3}\right), 35.88,35.93(\mathrm{CH}), 37.72,37.81(\mathrm{CH}), 39.73,39.75,39.81$, $39.84\left(2 \times \mathrm{CH}_{2}\right), 45.87,45.90\left(\mathrm{CH}_{2}\right), 47.41,47.60\left(\mathrm{CH}_{2}\right), 66.57\left(\mathrm{C}_{\mathrm{q}}\right), 70.11$ $\left(\mathrm{CH}_{2}\right), 205.82,206.03\left(\mathrm{C}_{\mathrm{q}}\right), 215.86,215.94\left(\mathrm{C}_{\mathrm{q}}\right)$

ESI-MS: $\quad 465\left(\mathrm{M}^{+}+\mathrm{H}\right), 341\left(\mathrm{M}^{+}-\mathrm{C}_{3} \mathrm{H}_{5} \mathrm{OS}_{2}-\mathrm{H}_{2}\right)$.

EI-HRMS: $\quad \mathrm{C}_{20} \mathrm{H}_{36} \mathrm{O}_{2} \mathrm{~S}_{4} \mathrm{Si}$, calcd.: 464.1367, found: 464.1321 . $\mathrm{C}_{20} \mathrm{H}_{36} \mathrm{O}_{2} \mathrm{~S}_{4} \mathrm{Si}(464.85)$ 

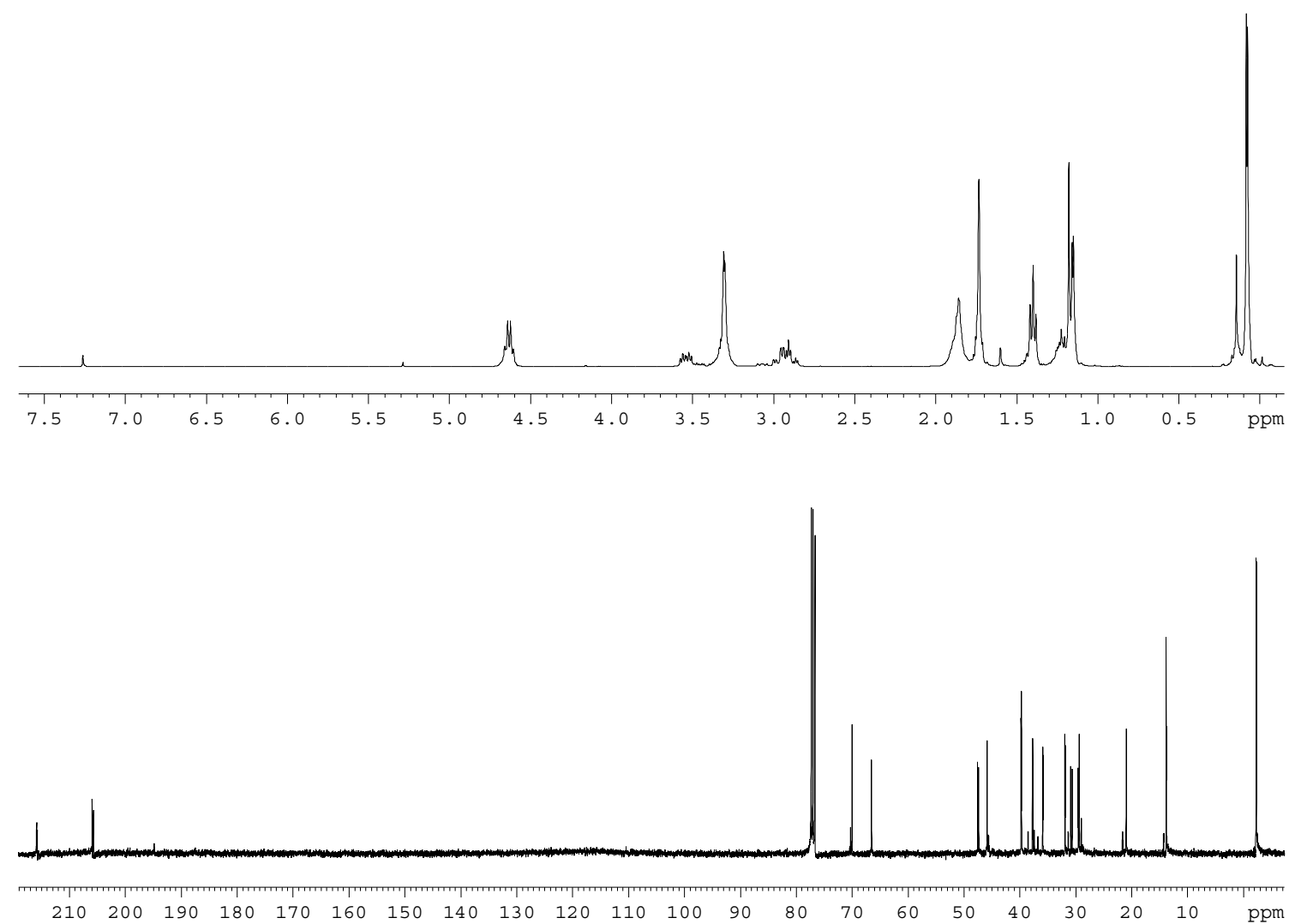

(E)-1-\{(1S,3R)-2,2-Dimethyl-3-[2-(2-methyl-[1,3]dithiolan-2-yl)-ethyl]-cyclopropyl\}-3trimethylsilanyl-propenone (35)

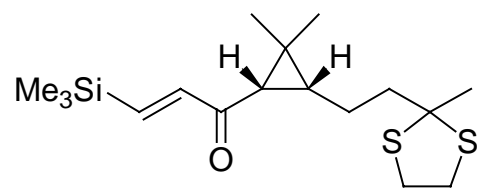

35

A solution of $34(25 \mathrm{mg}, 0.05 \mathrm{mmol})$ and DBU (12 mg, $0.08 \mathrm{mmol})$ in dichloromethane (3 $\mathrm{mL}$ ) was stirred under argon for $4 \mathrm{~h}$. The solution of the crude product was washed with satd. ammonium chloride and brine and dried over $\mathrm{Na}_{2} \mathrm{SO}_{4}$. Concentration and purification by column chromatography (1:20 EtOAc-hexanes) gave 35 (15 mg, $0.04 \mathrm{mmol}, 80 \%)$. Complete separation from bis-xanthate byproducts proved especially difficult in this case.

$$
\begin{array}{ll}
R_{\mathrm{f}}: & 0.70(1: 20 \text { EtOAc-hexanes }) \\
\alpha_{\mathrm{D}}: & +49^{\circ}\left(\mathrm{c}=1.0 \text { in } \mathrm{CHCl}_{3}\right) \\
\mathrm{IR}: & \left(\mathrm{CCl}_{4}\right): \mathrm{v} ;^{\sim}=2958(\mathrm{~s}), 2924(\mathrm{~s}), 1673(\mathrm{~m}), 1639(\mathrm{w}), 1450(\mathrm{~m}), 1416(\mathrm{~m}), \\
& 1376(\mathrm{~m}), 1250(\mathrm{~s}, \mathrm{C}-\mathrm{S}), 1205(\mathrm{~s}), 1059(\mathrm{~s}, \mathrm{C}=\mathrm{S}), 995(\mathrm{~m}) \mathrm{cm}^{-1} .
\end{array}
$$


${ }^{1} \mathrm{H}-\mathrm{NMR}: \quad\left(400 \mathrm{MHz}, \mathrm{CDCl}_{3}\right): \delta=0.14(\mathrm{~s}, 9 \mathrm{H}), 1.22(\mathrm{~s}, 6 \mathrm{H}), 1.33-1.39(\mathrm{~m}, 1 \mathrm{H}), 1.75$ $(\mathrm{s}, 3 \mathrm{H}), 1.85-1.93(\mathrm{~m}, 4 \mathrm{H}), 2.00\left(\mathrm{~d},{ }^{3} \mathrm{~J}=8.8 \mathrm{~Hz}, 1 \mathrm{H}\right), 3.28-3.35(\mathrm{~m}, 4 \mathrm{H})$, $6.56\left(\mathrm{~d},{ }^{3} J=18.8 \mathrm{~Hz}, 1 \mathrm{H}\right), 6.93\left(\mathrm{~d},{ }^{3} J=18.8 \mathrm{~Hz}, 1 \mathrm{H}\right)$.

${ }^{13} \mathrm{C}-\mathrm{NMR}: \quad\left(100.7 \mathrm{MHz}, \mathrm{CDCl}_{3}\right): \delta=-1.71\left(3 \times \mathrm{CH}_{3}\right), 13.98\left(\mathrm{CH}_{3}\right), 21.32\left(\mathrm{CH}_{2}\right), 29.49$ $\left(\mathrm{CH}_{3}\right), 29.85\left(\mathrm{C}_{\mathrm{q}}\right), 31.90\left(\mathrm{CH}_{3}\right), 34.82(\mathrm{CH}), 37.92(\mathrm{CH}), 39.71\left(\mathrm{CH}_{2}\right), 39.78$ $\left(\mathrm{CH}_{2}\right), 45.92\left(\mathrm{CH}_{2}\right), 66.66\left(\mathrm{C}_{\mathrm{q}}\right), 143.58(\mathrm{CH}), 144.74(\mathrm{CH}), 198.04\left(\mathrm{C}_{\mathrm{q}}\right)$.

ESI-MS: $359\left(\mathrm{M}^{+}+\mathrm{NH}_{3}\right), 343\left(\mathrm{M}^{+}+\mathrm{H}\right), 342\left(\mathrm{M}^{+}\right)$.

FAB-HRMS: $\quad \mathrm{C}_{17} \mathrm{H}_{31} \mathrm{OS}_{2} \mathrm{Si}^{+}$, calcd.: 343.1586, found: 343.1572 . $\mathrm{C}_{17} \mathrm{H}_{30} \mathrm{OS}_{2} \mathrm{Si}(342.64)$
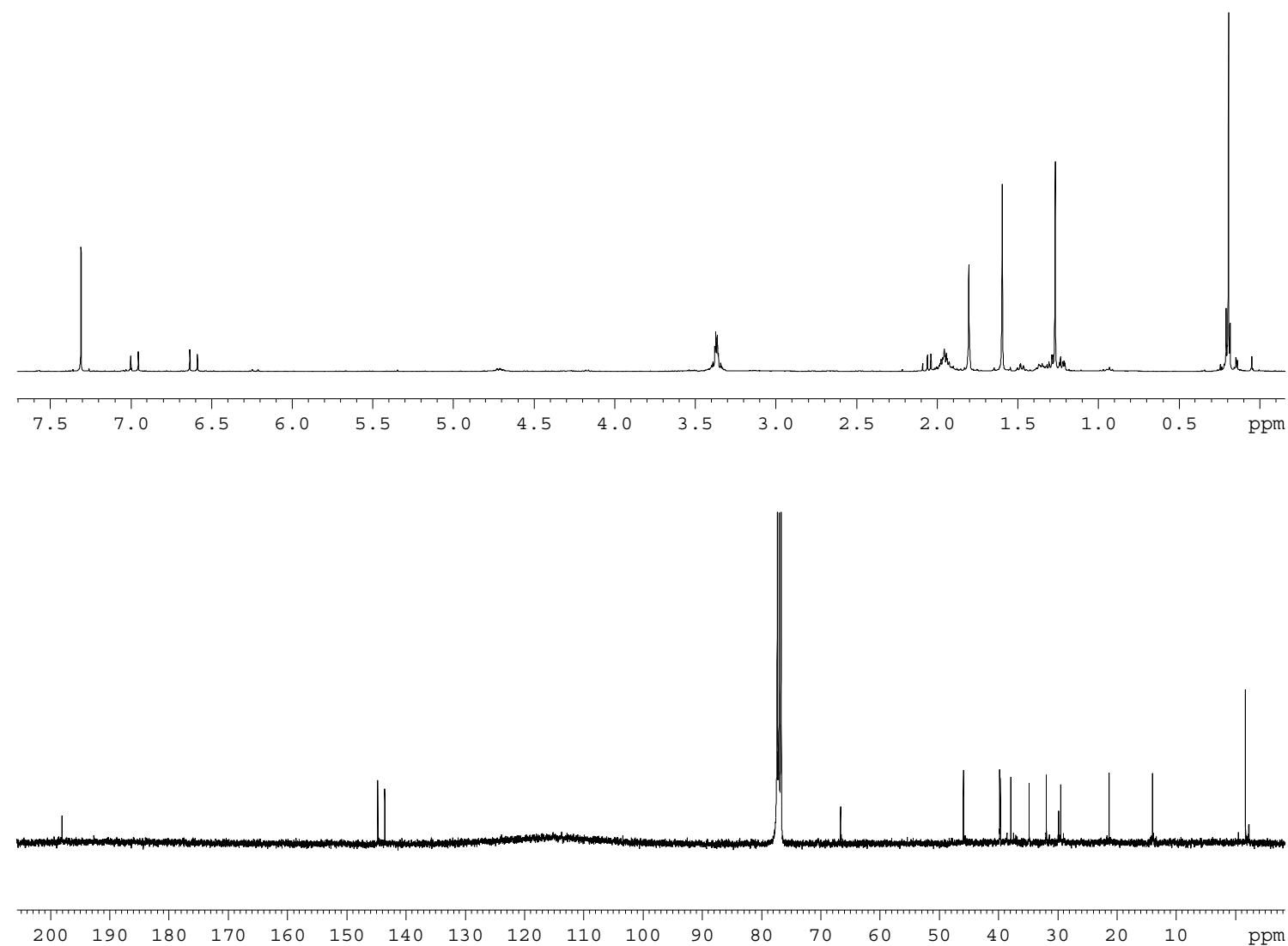


\section{Dithiocarbonic acid ethyl ester (1-phenyl-cyclopropylacyl) ester (36)}

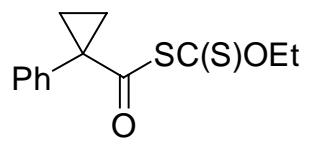

36

To a solution of 1-phenyl-cyclopropanecarboxylic acid $(1.00 \mathrm{~g}, 5.53 \mathrm{mmol})$ in acetone (15 $\mathrm{mL})$ at $0^{\circ} \mathrm{C}$ was added ethyl xanthic acid potassium salt $(0.84 \mathrm{~g}, 5.24 \mathrm{mmol})$. After stirring for $15 \mathrm{~min}$ at $0^{\circ} \mathrm{C}$ the solvent was removed under reduced pressure at $0-10^{\circ} \mathrm{C}$. The residue was dissolved in dichloromethane, washed with water and brine and dried over $\mathrm{Na}_{2} \mathrm{SO}_{4}$. Evaporation of the solvent gave acyl xanthate $\mathbf{3 6}$ (1.34 g, $5.03 \mathrm{mmol}, 96 \%)$

$R_{\mathrm{f}}: \quad 0.80(1: 10$ EtOAc-hexanes $)$

IR: $\quad\left(\mathrm{CCl}_{4}\right): \mathrm{v} ;^{\sim}=1710(\mathrm{~s}), 1697(\mathrm{~s}), 1257(\mathrm{~s}, \mathrm{C}-\mathrm{S}), 1039(\mathrm{~s}, \mathrm{C}=\mathrm{S}), 963(\mathrm{~s}) \mathrm{cm}^{-1}$. ${ }^{1} \mathrm{H}-\mathrm{NMR}: \quad\left(400 \mathrm{MHz}, \mathrm{CDCl}_{3}\right): \delta=1.35\left(\mathrm{dd},{ }^{3} J=7.2 \mathrm{~Hz},{ }^{2} J=3.6 \mathrm{~Hz}, 2 \mathrm{H}\right), 1.46\left(\mathrm{t},{ }^{3} J=\right.$ $7.0 \mathrm{~Hz}, 3 \mathrm{H}), 1.81\left(\mathrm{dd},{ }^{3} J=7.2 \mathrm{~Hz},{ }^{2} J=3.6 \mathrm{~Hz}, 2 \mathrm{H}\right), 4.64\left(\mathrm{t},{ }^{3} J=7.0 \mathrm{~Hz}\right.$, 2H), 7.36-7.38 (m, 3H), 7.44-7.46 (m, 2H).

${ }^{13} \mathrm{C}-\mathrm{NMR}: \quad\left(100.7 \mathrm{MHz}, \mathrm{CDCl}_{3}\right): \delta=13.43\left(\mathrm{CH}_{3}\right), 19.83\left(2 \times \mathrm{CH}_{2}\right), 38.06\left(\mathrm{C}_{\mathrm{q}}\right), 70.84$ $\left(\mathrm{CH}_{2}\right), 128.55(2 \times \mathrm{CH}), 128.84(\mathrm{CH}), 132.18(2 \times \mathrm{CH}), 136.46\left(\mathrm{C}_{\mathrm{q}}\right), 194.20$ $\left(\mathrm{C}_{\mathrm{q}}\right), 204.41\left(\mathrm{C}_{\mathrm{q}}\right)$.

ESI-MS: $\quad 284\left(\mathrm{M}^{+}+\mathrm{NH}_{4}\right), 267\left(\mathrm{M}^{+}+\mathrm{H}\right)$.

$\mathrm{C}_{13} \mathrm{H}_{14} \mathrm{O}_{2} \mathrm{~S}_{2}(266.38)$

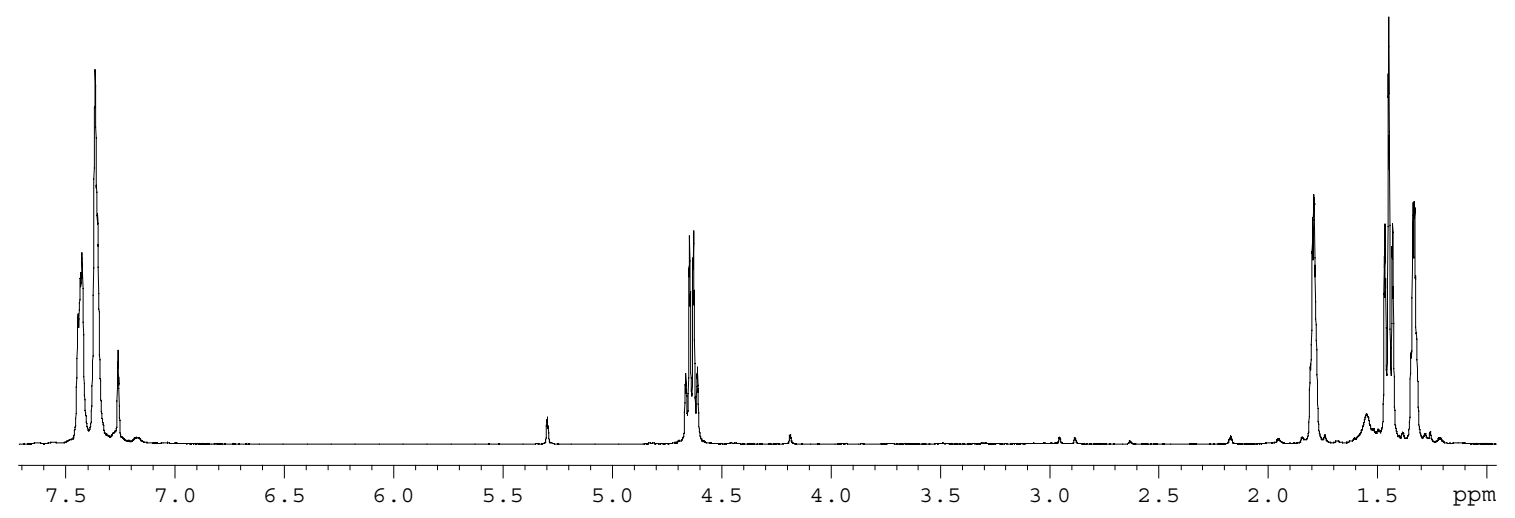




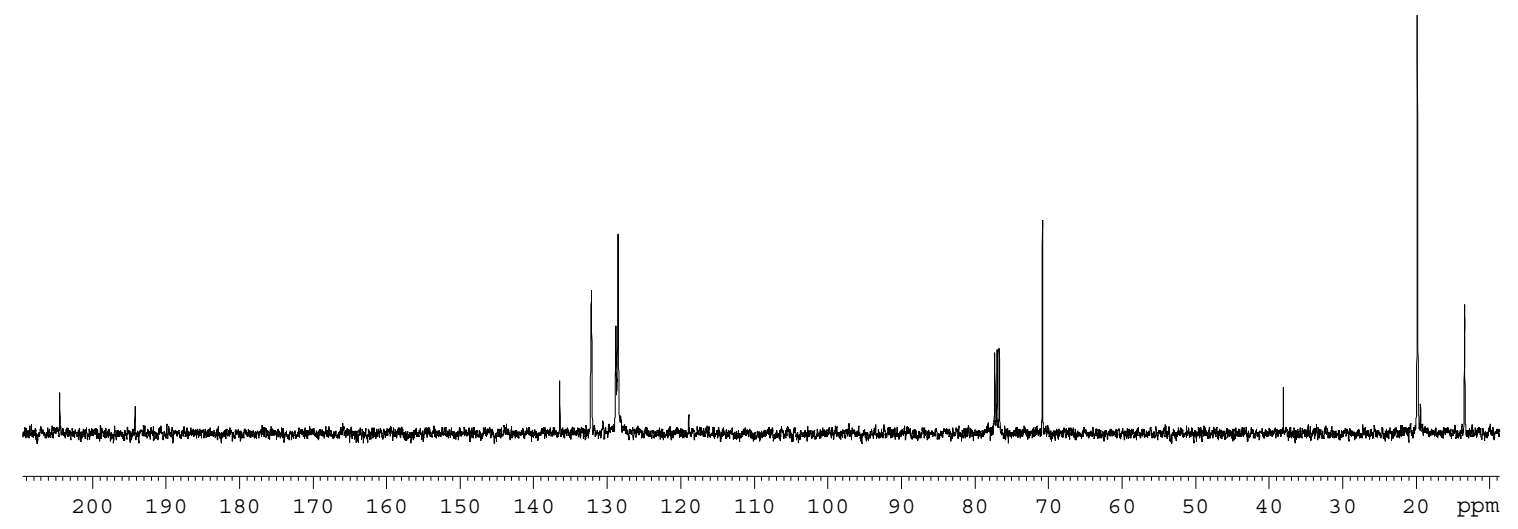

Dithiocarbonic acid ethyl ester (1-phenyl-cyclopropyl) ester (39)

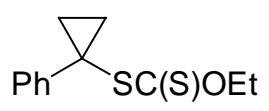

39

A solution of xanthate $36(131 \mathrm{mg}, 0.49 \mathrm{mmol})$ in toluene $(1.5 \mathrm{~mL})$ was exposed to visible light and refluxed for $4 \mathrm{~h}$ under argon. The solvent was removed under reduced pressure to give 39 (>85\% yield). Further purification is possible by column chromatography (1:20 EtOAc-hexanes).

$R_{\mathrm{f}}: \quad 0.85(1: 10$ EtOAc-hexanes $)$

IR: $\quad\left(\mathrm{CCl}_{4}\right): \mathrm{v}^{\sim}=1222(\mathrm{~s}, \mathrm{C}-\mathrm{S}), 1049(\mathrm{~s}, \mathrm{C}=\mathrm{S}) \mathrm{cm}^{-1}$.

${ }^{1} \mathrm{H}-\mathrm{NMR}: \quad\left(400 \mathrm{MHz}, \mathrm{CDCl}_{3}\right): \delta=1.40\left(\mathrm{t},{ }^{3} \mathrm{~J}=6.8 \mathrm{~Hz}, 3 \mathrm{H}\right), 1.46\left(\mathrm{dd},{ }^{3} J=10.0 \mathrm{~Hz},{ }^{2} J\right.$ $=2.0 \mathrm{~Hz}, 2 \mathrm{H}), 1.47\left(\mathrm{dd},{ }^{3} J=10.0 \mathrm{~Hz},{ }^{2} J=2.0 \mathrm{~Hz}, 2 \mathrm{H}\right), 4.60\left(\mathrm{t},{ }^{3} J=6.8 \mathrm{~Hz}\right.$, 2H), 7.19-7.26 (m, 1H), 7.27-7.33 (m, 2H), 7.45-7.50 (m, 2H).

${ }^{13} \mathrm{C}-\mathrm{NMR}: \quad\left(100.7 \mathrm{MHz}, \mathrm{CDCl}_{3}\right): \delta=13.72\left(\mathrm{CH}_{3}\right), 17.41\left(2 \times \mathrm{CH}_{2}\right), 30.51\left(\mathrm{C}_{\mathrm{q}}\right), 69.60$ $\left(\mathrm{CH}_{2}\right), 126.76(2 \times \mathrm{CH}), 128.02(2 \times \mathrm{CH}), 128.30(\mathrm{CH}), 142.37\left(\mathrm{C}_{\mathrm{q}}\right), 213.71$ $\left(\mathrm{C}_{\mathrm{q}}\right)$.

ESI-MS: $\quad 239\left(\mathrm{M}^{+}+\mathrm{H}\right)$.

EI-HRMS: $\quad \mathrm{C}_{12} \mathrm{H}_{14} \mathrm{OS}_{2}$, calcd.: 238.0486, found: 238.0485.

$\mathrm{C}_{12} \mathrm{H}_{14} \mathrm{OS}_{2}$ (238.37) 

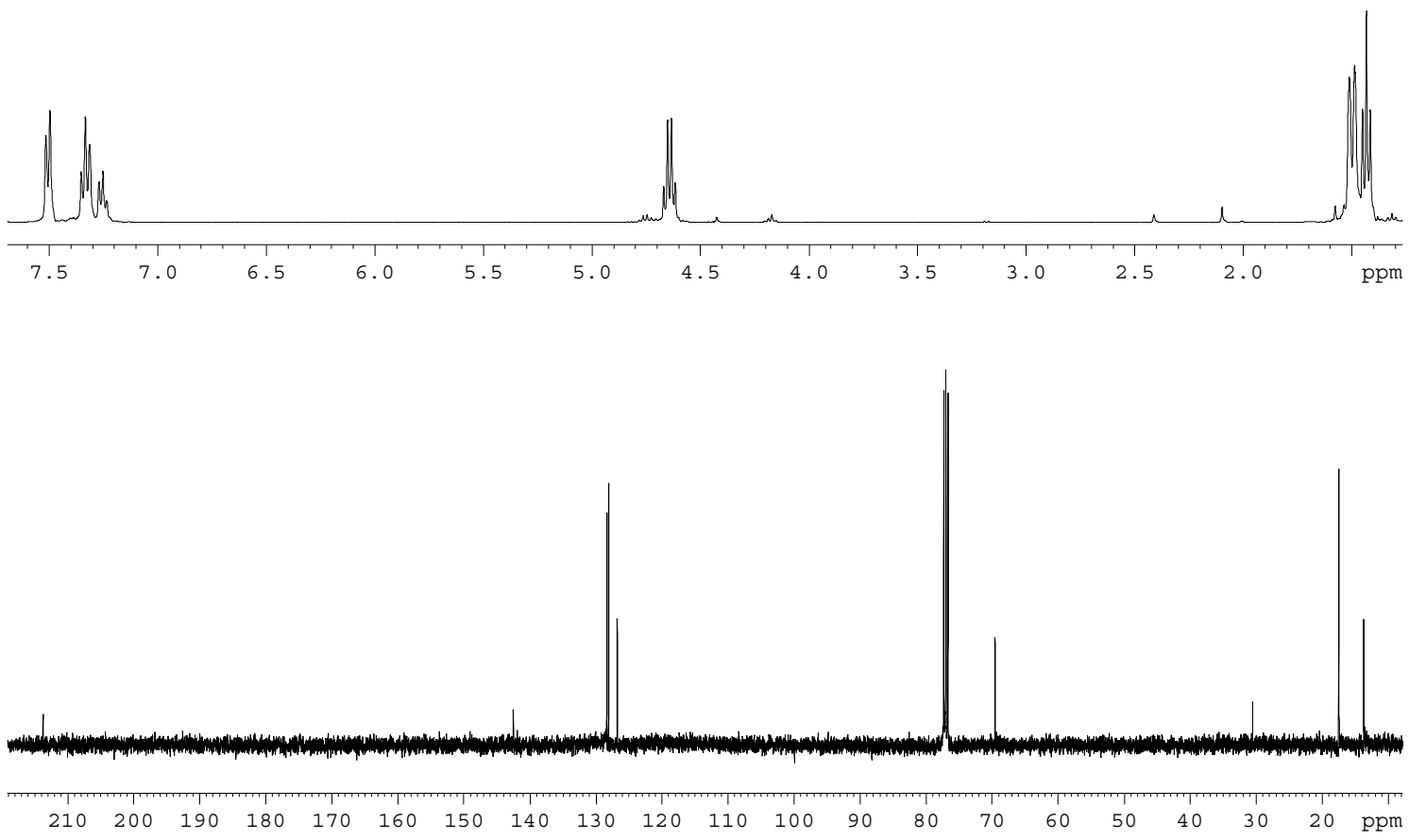

Acetic acid 2-ethoxythiocarbonylsulfanyl-4-oxo-4-(1-phenyl-cyclopropyl)-butyl ester (40)<smiles>CCO[Si]C(COC(C)=O)CC(=O)C1(c2ccccc2)CC1</smiles>

A solution of xanthate $36(152 \mathrm{mg}, 0.57 \mathrm{mmol})$ in allyl acetate $(1 \mathrm{~mL})$ was exposed to visible light and refluxed for $1.5 \mathrm{~h}$. Concentration and purification by column chromatography (1:10 EtOAc-hexanes) gave $\mathbf{4 0}$ (128 mg, $0.35 \mathrm{mmol}, 61 \%)$ and also decarbonylated xanthate 39 (30 $\mathrm{mg}, 0.13 \mathrm{mmol}, 22 \%)$.

$R_{\mathrm{f}}: \quad 0.35(1: 10$ EtOAc-hexanes $)$

IR: $\quad\left(\mathrm{CCl}_{4}\right): \mathrm{v}^{\sim}{ }^{\sim}=1748(\mathrm{~s}), 1697(\mathrm{~s}), 1228(\mathrm{~s}, \mathrm{C}-\mathrm{S}), 1052(\mathrm{~s}, \mathrm{C}=\mathrm{S}), 1024(\mathrm{~m}) \mathrm{cm}^{-}$ 1 .

${ }^{1} \mathrm{H}-\mathrm{NMR}: \quad\left(400 \mathrm{MHz}, \mathrm{CDCl}_{3}\right): \delta=1.20\left(\mathrm{dd},{ }^{3} J=6.4 \mathrm{~Hz},{ }^{2} J=2.8 \mathrm{~Hz}, 2 \mathrm{H}\right), 1.38\left(\mathrm{t},{ }^{3} J=\right.$ $7.2 \mathrm{~Hz}, 3 \mathrm{H}), 1.64\left(\mathrm{dd},{ }^{3} J=6.4 \mathrm{~Hz},{ }^{2} J=2.8 \mathrm{~Hz}, 2 \mathrm{H}\right), 2.06(\mathrm{~s}, 3 \mathrm{H}), 2.72\left(\mathrm{~d},{ }^{3} J\right.$ $=6.0 \mathrm{~Hz}, 2 \mathrm{H}), 4.12-4.17(\mathrm{~m}, 1 \mathrm{H}), 4.26-4.31(\mathrm{~m}, 2 \mathrm{H}), 4.60\left(\mathrm{t},{ }^{3} J=7.2 \mathrm{~Hz}\right.$, 2H), 7.30-7.38 (m, 5H).

${ }^{13} \mathrm{C}-\mathrm{NMR}: \quad\left(100.7 \mathrm{MHz}, \mathrm{CDCl}_{3}\right): \delta=13.58\left(\mathrm{CH}_{3}\right), 19.19\left(2 \times \mathrm{CH}_{2}\right), 20.61\left(\mathrm{CH}_{3}\right), 37.28$ $\left(\mathrm{C}_{\mathrm{q}}\right), 42.09\left(\mathrm{CH}_{2}\right), 43.92(\mathrm{CH}), 64.48\left(\mathrm{CH}_{2}\right), 70.02\left(\mathrm{CH}_{2}\right), 127.64(\mathrm{CH})$, 
$128.70(2 \times \mathrm{CH}), 130.76(2 \times \mathrm{CH}), 139.84\left(\mathrm{C}_{\mathrm{q}}\right), 170.33\left(\mathrm{C}_{\mathrm{q}}\right), 206.96\left(\mathrm{C}_{\mathrm{q}}\right)$,

$212.64\left(\mathrm{C}_{\mathrm{q}}\right)$.

ESI-MS: $\quad 384\left(\mathrm{M}^{+}+\mathrm{NH}_{4}\right), 367\left(\mathrm{M}^{+}+\mathrm{H}\right)$.

FAB-HRMS: $\quad \mathrm{C}_{18} \mathrm{H}_{22} \mathrm{O}_{4} \mathrm{~S}_{2} \mathrm{Na}^{+}$, calcd.: 389.0857, found: 389.0880.

$\mathrm{C}_{18} \mathrm{H}_{23} \mathrm{O}_{4} \mathrm{~S}_{2}{ }^{+}$, calcd.: 367.1038 , found: 367.1067 .

$\mathrm{C}_{18} \mathrm{H}_{22} \mathrm{O}_{4} \mathrm{~S}_{2}(366.50)$
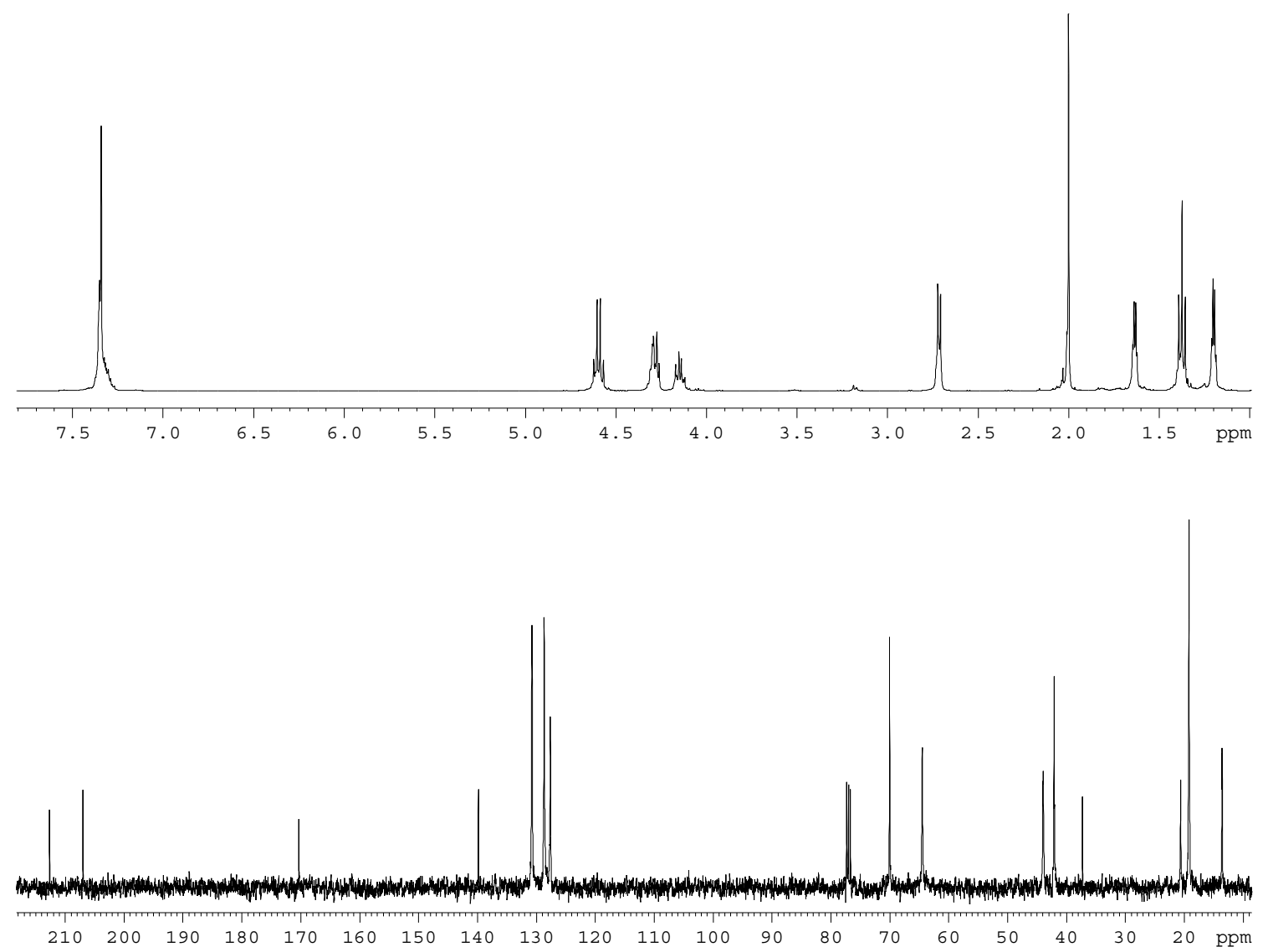

Dithiocarbonic acid ethyl ester [3-oxo-3-(1-phenyl-cyclopropyl)-1-trimethylsilanylpropyl] ester (41)

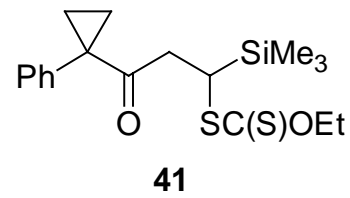

A solution of xanthate $36(135 \mathrm{mg}, 0.51 \mathrm{mmol})$ in vinyl trimethylsilane $(1.5 \mathrm{~mL})$ was exposed to visible light and refluxed for $45 \mathrm{~min}$. Concentration and purification by column chromatography (3:97 EtOAc-hexanes) gave 41 (162 mg, 0.44 mmol, 87\%). 
$R_{\mathrm{f}}:$

IR:

${ }^{1} \mathrm{H}-\mathrm{NMR}$ :

${ }^{13} \mathrm{C}-\mathrm{NMR}$ :

ESI-MS:

384

\subsection{5 (1:20 EtOAc-hexanes)}

$\left(\mathrm{CCl}_{4}\right): \mathrm{v}^{\sim}{ }^{2}=1697$ (s), 1219 (s, C-S), 1050 (s, C=S) cm $\mathrm{cm}^{-1}$.

$\left(400 \mathrm{MHz}, \mathrm{CDCl}_{3}\right): \delta=0.01(\mathrm{~s}, 9 \mathrm{H}), 1.12\left(\mathrm{ddd},{ }^{3} J=8.4 \mathrm{~Hz}, J=6.0 \mathrm{~Hz}, J=\right.$ $2.4 \mathrm{~Hz}, 1 \mathrm{H}), 1.17\left(\mathrm{ddd},{ }^{3} J=8.4 \mathrm{~Hz}, J=6.0 \mathrm{~Hz}, J=2.4 \mathrm{~Hz}, 1 \mathrm{H}\right), 1.36\left(\mathrm{t},{ }^{3} J=\right.$ $7.0 \mathrm{~Hz}, 3 \mathrm{H}), 1.55\left(\mathrm{ddd},{ }^{3} J=10.0 \mathrm{~Hz}, J=6.0 \mathrm{~Hz}, J=2.4 \mathrm{~Hz}, 1 \mathrm{H}\right), 1.62$ (ddd, $\left.{ }^{3} J=10.0 \mathrm{~Hz}, J=6.0 \mathrm{~Hz}, J=2.4 \mathrm{~Hz}, 1 \mathrm{H}\right), 2.63\left(\mathrm{dd},{ }^{2} J=18.8 \mathrm{~Hz},{ }^{3} J=4.4\right.$ $\mathrm{Hz}, 1 \mathrm{H}), 2.79\left(\mathrm{dd},{ }^{2} J=18.8 \mathrm{~Hz},{ }^{3} J=7.0 \mathrm{~Hz}, 1 \mathrm{H}\right), 3.42\left(\mathrm{dd},{ }^{3} J=7.0 \mathrm{~Hz},{ }^{3} J=\right.$ $4.4 \mathrm{~Hz}, 1 \mathrm{H}), 4.59$ (q, $\left.{ }^{3} J=7.0 \mathrm{~Hz}, 2 \mathrm{H}\right), 7.28-7.34(\mathrm{~m}, 5 \mathrm{H})$.

$\left(100.7 \mathrm{MHz}, \mathrm{CDCl}_{3}\right): \delta=-2.25\left(3 \times \mathrm{CH}_{3}\right), 13.73\left(\mathrm{CH}_{3}\right), 18.95\left(\mathrm{CH}_{2}\right), 19.06$ $\left(\mathrm{CH}_{2}\right), 30.59(\mathrm{CH}), 37.09\left(\mathrm{C}_{\mathrm{q}}\right), 43.43\left(\mathrm{CH}_{2}\right), 69.98\left(\mathrm{CH}_{2}\right), 127.55(\mathrm{CH})$, $128.68(2 \times \mathrm{CH}), 130.83(2 \times \mathrm{CH}), 140.43\left(\mathrm{C}_{\mathrm{q}}\right), 208.26\left(\mathrm{C}_{\mathrm{q}}\right), 216.00\left(\mathrm{C}_{\mathrm{q}}\right)$. $384\left(\mathrm{M}^{+}+\mathrm{NH}_{4}\right), 367\left(\mathrm{M}^{+}+\mathrm{H}\right)$.

\section{$\mathrm{C}_{18} \mathrm{H}_{26} \mathrm{O}_{2} \mathrm{~S}_{2} \mathrm{Si}$ (366.62)}
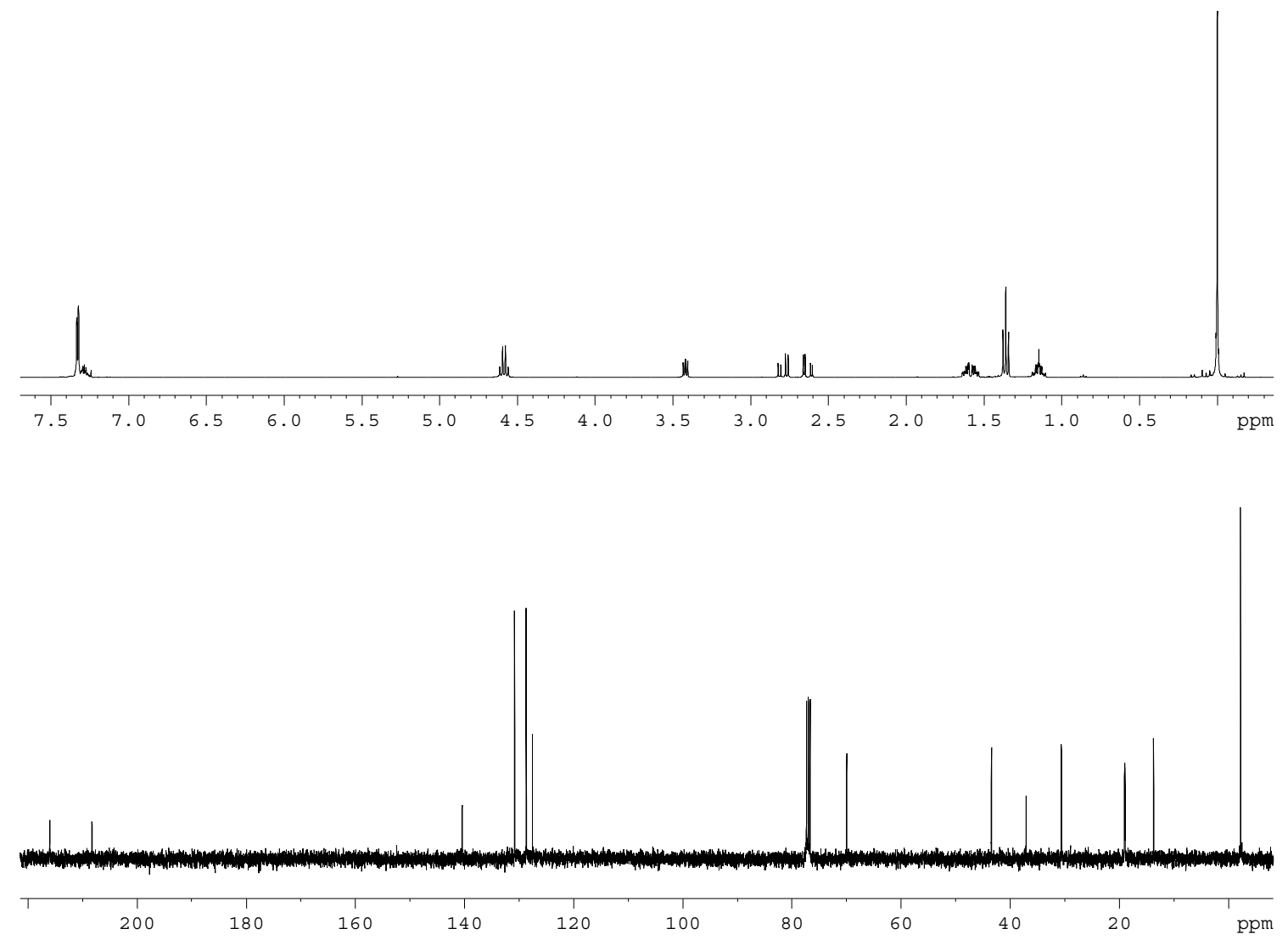


\section{(E)-1-(1-Phenyl-cyclopropyl)-3-trimethylsilanyl-propenone (42)}

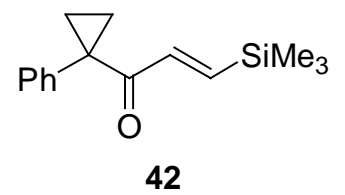

A solution of xanthate $36(0.53 \mathrm{~g}, 2.0 \mathrm{mmol})$ in vinyl trimethylsilane $(0.8 \mathrm{~mL})$ was exposed to visible light and refluxed for $7 \mathrm{~h}$ under argon. The solvent was removed under reduced pressure and the residue was dissolved in dichloromethane $(10 \mathrm{~mL})$. After the addition of DBU $(0.38 \mathrm{~mL}, 0.38 \mathrm{~g}, 2.5 \mathrm{mmol})$ the reaction mixture was stirred under argon for $1.5 \mathrm{~h}$. The solution of the crude product was washed with satd. ammonium chloride, followed by brine and dried over $\mathrm{Na}_{2} \mathrm{SO}_{4}$. Purification by column chromatography (4:96 $\mathrm{Et}_{2} \mathrm{O}$-hexanes) and sublimation (ca. $0.1 \mathrm{mbar}$ ) gave $42(347 \mathrm{mg}, 1.42 \mathrm{mmol}, 71 \%)$. Partial isomerization occured while purification.

Yield: $\quad 71 \%$

$R_{\mathrm{f}}: \quad 0.5\left(3: 97 \mathrm{Et}_{2} \mathrm{O}\right.$-hexanes $)$

IR: $\quad\left(\mathrm{CCl}_{4}\right): \mathrm{v}^{\sim}=1675(\mathrm{~s}), 1263(\mathrm{~s}), 1250(\mathrm{~s}), 1070(\mathrm{~s}) \mathrm{cm}^{-1}$.

${ }^{1} \mathrm{H}-\mathrm{NMR}: \quad\left(400 \mathrm{MHz}, \mathrm{CDCl}_{3}\right): \delta=0.01(\mathrm{~s}, 9 \mathrm{H}), 1.27\left(\mathrm{dd},{ }^{3} J=7.0 \mathrm{~Hz},{ }^{2} J=3.6 \mathrm{~Hz}\right.$, $2 \mathrm{H}), 1.68\left(\mathrm{dd},{ }^{3} J=7.0 \mathrm{~Hz},{ }^{2} J=3.6 \mathrm{~Hz}, 2 \mathrm{H}\right), 6.47\left(\mathrm{~d},{ }^{3} J=18.8 \mathrm{~Hz}, 1 \mathrm{H}\right), 7.06$ $\left(\mathrm{d},{ }^{3} J=18.8 \mathrm{~Hz}, 1 \mathrm{H}\right), 7.32-7.37(\mathrm{~m}, 5 \mathrm{H})$.

${ }^{13} \mathrm{C}-\mathrm{NMR}: \quad\left(100.7 \mathrm{MHz}, \mathrm{CDCl}_{3}\right): \delta=-2.03\left(3 \times \mathrm{CH}_{3}\right), 18.96\left(2 \times \mathrm{CH}_{2}\right), 36.98\left(\mathrm{C}_{\mathrm{q}}\right)$, $127.29(\mathrm{CH}), 128.49(2 \times \mathrm{CH}), 130.57(2 \times \mathrm{CH}), 138.24(\mathrm{CH}), 140.36\left(\mathrm{C}_{\mathrm{q}}\right)$, $146.13(\mathrm{CH}), 198.59\left(\mathrm{C}_{\mathrm{q}}\right)$.

ESI-MS: $\quad 262\left(\mathrm{M}^{+}+\mathrm{NH}_{4}\right), 245\left(\mathrm{M}^{+}+\mathrm{H}\right)$.

FAB-HRMS: $\quad \mathrm{C}_{15} \mathrm{H}_{21} \mathrm{OSi}^{+}$, calcd.: 245.1352, found: 245.1386.

$\mathrm{C}_{15} \mathrm{H}_{20} \mathrm{OSi}(244.40)$ 


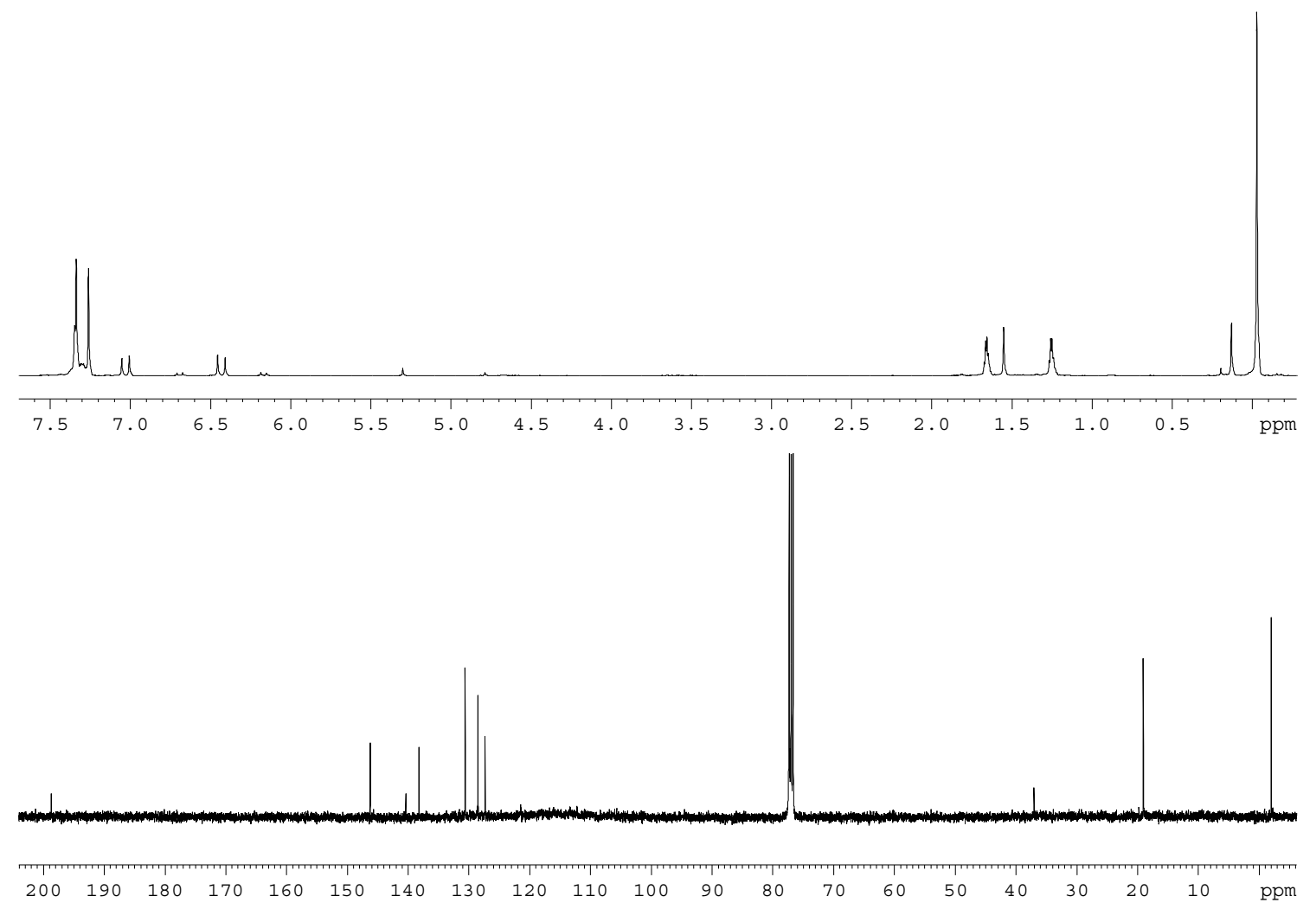

Acetic acid 2-ethoxythiocarbonylsulfanyl-3-(1-phenyl-cyclopropyl)-propyl ester (43)

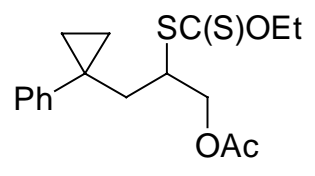

43

A solution of xanthate $39(88 \mathrm{mg}, 0.37 \mathrm{mmol})$ and allyl acetate $(0.12 \mathrm{~mL}, 111 \mathrm{mg}, 1.11$ mmol) in 1,2-dichloroethane $(0.5 \mathrm{~mL})$ was refluxed for 5 mins under argon. After the addition of DLP (15 mg, $0.04 \mathrm{mmol}, 10 \%)$ the mixture was refluxed for $3 \mathrm{~h}$ and two further portions of DLP were added after 1 and $2 \mathrm{~h}$ intervals. The reaction mixture was concentrated under reduced pressure and purified by column chromatography (1:20 to 1:4 EtOAc-hexanes) to give 43 (28 mg, $0.08 \mathrm{mmol}, 22 \%)$ and 44 (40 mg, $0.09 \mathrm{mmol}, 25 \%)$.

$R_{\mathrm{f}}: \quad 0.8(1: 10$ EtOAc-hexanes $)$

IR: $\quad\left(\mathrm{CCl}_{4}\right): \mathrm{v}^{\sim}{ }^{\sim}=1747(\mathrm{~s}), 1225(\mathrm{~s}, \mathrm{C}-\mathrm{S}), 1052(\mathrm{~s}, \mathrm{C}=\mathrm{S}) \mathrm{cm}^{-1}$.

${ }^{1} \mathrm{H}-\mathrm{NMR}: \quad\left(400 \mathrm{MHz}, \mathrm{CDCl}_{3}\right): \delta=0.65-0.71(\mathrm{~m}, 1 \mathrm{H}), 0.77-0.83(\mathrm{~m}, 1 \mathrm{H}), 0.86-0.97$ $(\mathrm{m}, 2 \mathrm{H}), 1.35\left(\mathrm{t},{ }^{3} J=7.2 \mathrm{~Hz}, 3 \mathrm{H}\right), 1.95\left(\mathrm{dd},{ }^{2} J=14.6 \mathrm{~Hz},{ }^{3} J=7.6 \mathrm{~Hz}, 1 \mathrm{H}\right)$, 
$2.03(\mathrm{~s}, 3 \mathrm{H}), 2.11\left(\mathrm{dd},{ }^{2} J=14.6 \mathrm{~Hz},{ }^{3} J=7.6 \mathrm{~Hz}, 1 \mathrm{H}\right), 3.77-3.84(\mathrm{~m}, 1 \mathrm{H})$,

4.22 (“d”, $\left.{ }^{3} J=4.8 \mathrm{~Hz}, 2 \mathrm{H}\right), 4.58\left(\mathrm{q},{ }^{3} J=7.2 \mathrm{~Hz}, 2 \mathrm{H}\right), 7.20-7.38(\mathrm{~m}, 5 \mathrm{H})$.

${ }^{13} \mathrm{C}-\mathrm{NMR}: \quad\left(100.7 \mathrm{MHz}, \mathrm{CDCl}_{3}\right): \delta=12.43\left(\mathrm{CH}_{2}\right), 13.02\left(\mathrm{CH}_{2}\right), 13.32\left(\mathrm{CH}_{3}\right), 20.46$

$\left(\mathrm{CH}_{3}\right), 23.38\left(\mathrm{C}_{\mathrm{q}}\right), 40.63\left(\mathrm{CH}_{2}\right), 47.35(\mathrm{CH}), 64.88\left(\mathrm{CH}_{2}\right), 69.55\left(\mathrm{CH}_{2}\right)$, $126.25(\mathrm{CH}), 128.09(2 \times \mathrm{CH}), 128.84(2 \times \mathrm{CH}), 142.97\left(\mathrm{C}_{\mathrm{q}}\right), 170.36\left(\mathrm{C}_{\mathrm{q}}\right)$, $212.45\left(\mathrm{C}_{\mathrm{q}}\right)$.

ESI-MS: $\quad 356\left(\mathrm{M}^{+}+\mathrm{NH}_{4}\right), 339\left(\mathrm{M}^{+}+\mathrm{H}\right)$.

FAB-HRMS: $\quad \mathrm{C}_{17} \mathrm{H}_{23} \mathrm{O}_{3} \mathrm{~S}_{2}{ }^{+}$, calcd.: 399.1089, found: 339.1099 .

$\mathrm{C}_{17} \mathrm{H}_{22} \mathrm{O}_{3} \mathrm{~S}_{2}(338.49)$
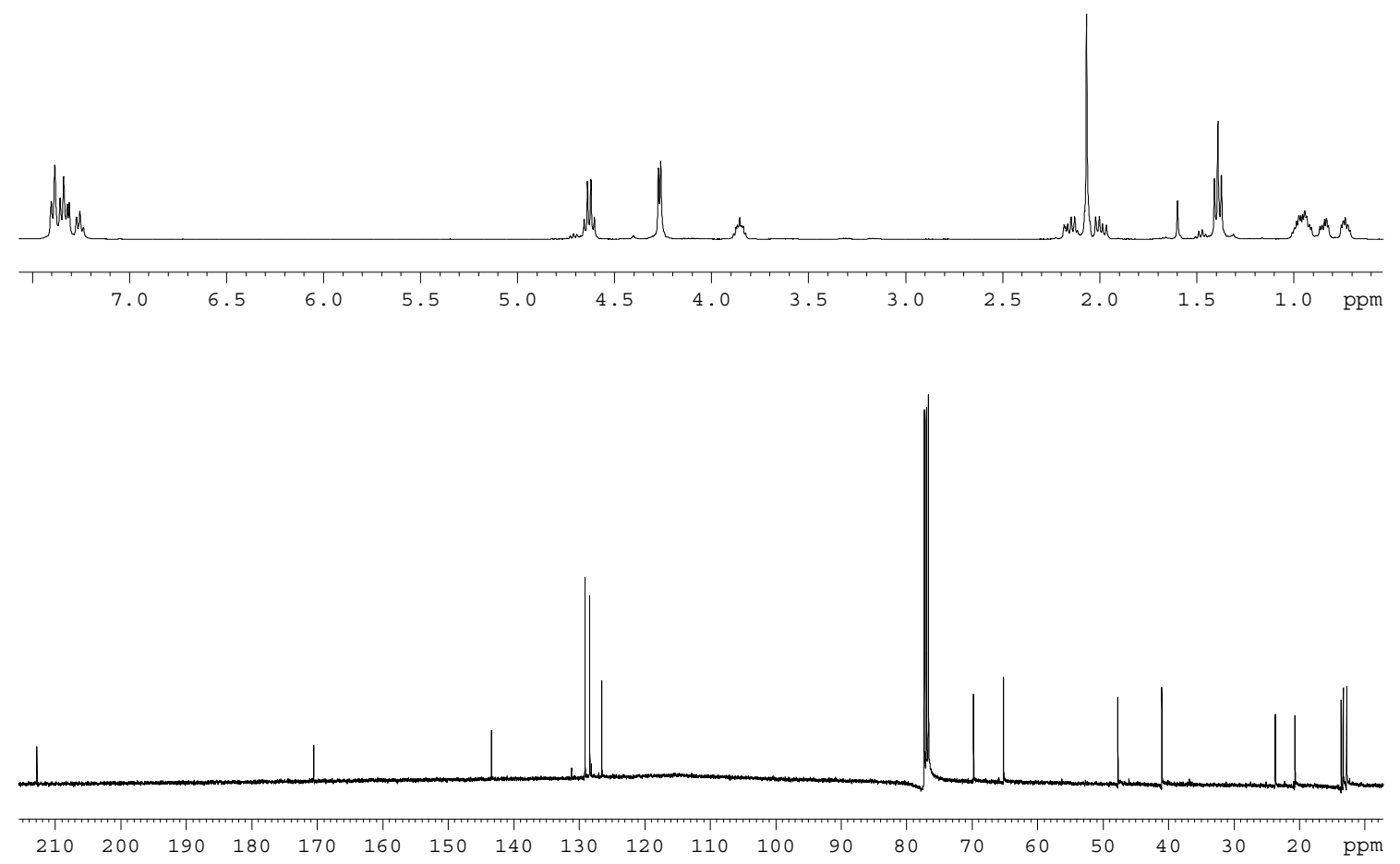

Acetic acid 5-acetoxy-4-ethoxythiocarbonylsulfanyl-2-(1-phenyl-cyclopropylmethyl)pentyl ester (44), mixture of diastereoisomers<smiles>CCOC(=O)SC(COC(C)=O)CC(COC(C)=O)CC1(c2ccccc2)CC1</smiles> 
$R_{\mathrm{f}}:$

IR:

${ }^{1} \mathrm{H}-\mathrm{NMR}$ :

${ }^{13}$ C-NMR:

ESI-MS:

$$
\begin{aligned}
& 456\left(\mathrm{M}^{+}+\mathrm{NH}_{4}\right), 439\left(\mathrm{M}^{+}+\mathrm{H}\right) . \\
& \mathrm{C}_{22} \mathrm{H}_{30} \mathrm{O}_{5} \mathrm{~S}_{2}(438.60)
\end{aligned}
$$$$
213.05,213.17\left(\mathrm{C}_{\mathrm{q}}\right) \text {. }
$$
7.15-7.32 (m, 5H). $213.05,213.17\left(\mathrm{C}_{\mathrm{q}}\right)$. 1.80-1.96 (m, 2H), 2.08 (s, 6H), 3.90-4.17 (m, 5H), 4.58-4.68 (m, 2H),

$\left(100.7 \mathrm{MHz}, \mathrm{CDCl}_{3}\right): \delta=12.60,12.97,13.15,13.30\left(2 \times \mathrm{CH}_{2}\right), 13.76,13.81$ $\left(\mathrm{CH}_{3}\right), 20.77,20.80,20.92,20.96\left(2 \times \mathrm{CH}_{3}\right), 23.40,23.76\left(\mathrm{C}_{\mathrm{q}}\right), 32.73,33.15$ $\left(\mathrm{CH}_{2}\right)$, 33.66, $33.81(\mathrm{CH}), 41.23,42.17\left(\mathrm{CH}_{2}\right), 47.23,47.38(\mathrm{CH})$, 65.86, 65.91, 66.08, $66.57\left(2 \times \mathrm{CH}_{2}\right), 70.17\left(\mathrm{CH}_{2}\right), 126.21,126.30(\mathrm{CH}), 128.36$, 128.44, 128.64, $128.88(4 \times \mathrm{CH}), 144.06,144.16\left(\mathrm{C}_{\mathrm{q}}\right), 170.65,171.09\left(\mathrm{C}_{\mathrm{q}}\right)$,
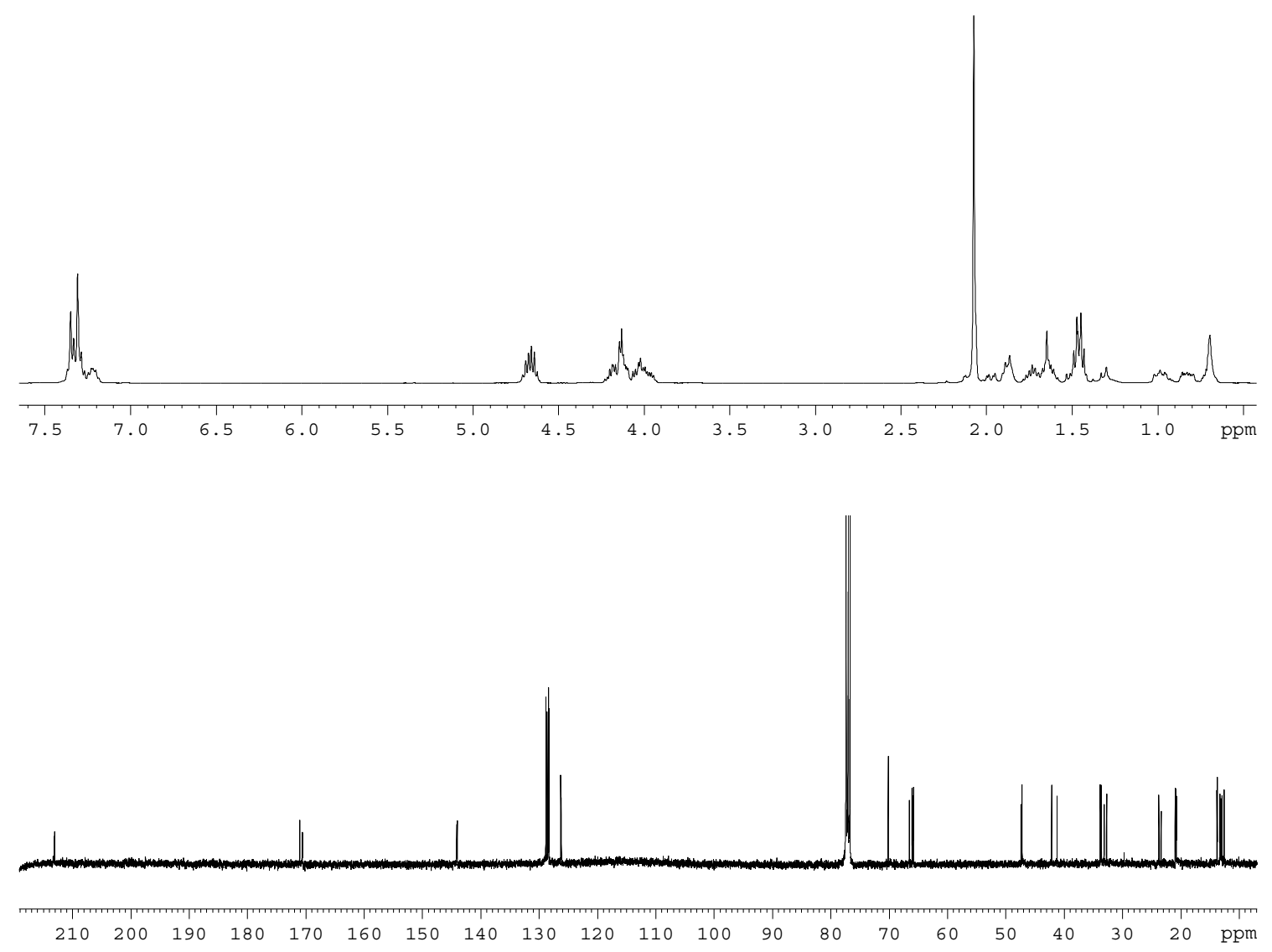


\section{References:}

1. R. A. Aitken, H. R. Cooper, A. P. Mehrotra, J. Chem. Soc., Perkin Trans. 1 1996, $475-484$.

2. S. Collet, P. Bauchat, R. Danion-Bougot, D. Danion, Tetrahedron: Asymmetry 1998, 9, 2121-2132; S. D. Abbott, P. Lane-Bell, K. P. S. Sidhu, J. C. Vederas, J. Am. Chem. Soc. 1994, 116, 6513-6520.

3. J. Haddow, C. J. Suckling, H. C. S. Woood, J. Chem. Soc., Perkin Trans. 1 1989, 1297-1304.

Due to the low yields reported for the preparation of acid chloride 14, we were happy to find a more convenient modified procedure.

Therefore 1-aminocyclopropane carboxylic acid hydrochloride was obtained from the described precursor by heating in conc. $\mathrm{HCl}$ at $70^{\circ} \mathrm{C}$ for $5 \mathrm{~h}$. The cooled solution was extracted three times with diethylether and was then concentrated and dried to give the crude amino acid hydrochloride. The crude hydrochloride (2.00 g, $14.5 \mathrm{mmol})$, triethylamine $(6.0 \mathrm{~mL}, 4.4 \mathrm{~g}, 43.5 \mathrm{mmol})$ and toluene $(120 \mathrm{~mL})$ were placed in a DeanStark apparatus and heated under reflux for $1.5 \mathrm{~h}$. After the addition of phthalic anhydride (2.37 g, $16.0 \mathrm{mmol})$ heating was continued for $6 \mathrm{~h}$. The reaction mixture was concentrated, treated with $2 \mathrm{~N} \mathrm{HCl}$ and extracted several times with ethyl acetate. The combined extracts were washed with brine and dried over $\mathrm{Na}_{2} \mathrm{SO}_{4}$. Recrystallization from dichloromethane gave the phthaloyl-protected amino acid (2.68 g, $11.6 \mathrm{mmol}, 80 \%)$. Conversion to the acid chloride was achieved using the standard procedure involving oxalyl chloride and catalytic dimethylformamide in dichloromethane.

4. a) B. A. Cheskis, N. M. Ivanova, A. M. Moiseenkov, O. M. Nefedov, Bull. Acad. Sci. USSR, Div. Chem. Sci. 1990, 39, 1839-1849; b) A. M. Moiseenkov, B. A. Cheskis, N. M. Ivanova, O. M. Nefedov, J. Chem. Soc., Perkin Trans. 1 1991, 2639-2649; c) B. A. Cheskis, N. M. Ivanova, A. M. Moiseenkov, O. M. Nefedov, Bull. Acad. Sci. USSR, Div. Chem. Sci. 1991, 40, 1372-1380;

${ }^{1} \mathrm{H}$ NMR data of $\mathbf{3 0}$ is in full accord with the data reported in ref. $4 \mathrm{~b}$.

5. R. P. Hatch, J. Shringarpure, S. M. Weinreb, J. Org. Chem. 1978, 43, 4172-

6. J. G. Donkervoort, A. R. Gordon, C. Johnstone, W. J. Kerr, U. Lange, Tetrahedron 1996, $52,7391-7420$. 